\title{
Improving the Performance of Video Processing on Hadoop Clusters
}

by

\author{
Eihab Hadi SaatiAlsoruji
}

A Dissertation submitted to the Faculty of Graduate and Postdoctoral Affairs in partial fulfillment of the requirements for the degree of

\author{
Doctor of Philosophy \\ in
}

Computer Science

\author{
School of Computer Science \\ Carleton University \\ Ottawa, Ontario, Canada
}

2020

\author{
Copyright (C) \\ 2020 - Eihab Hadi SaatiAlsoruji
}


The undersigned recommend to

the Faculty of Graduate Studies and Research

acceptance of the Dissertation

\title{
Improving the Performance of Video Processing on Hadoop Clusters
}

\author{
Submitted by Eihab Hadi SaatiAlsoruji \\ in partial fulfilment of the requirements for the degree of \\ Doctor of Philosophy
}

Shikharesh Majumdar, Supervisor

Anil Maheshwari, Co-supervisor

Carleton University

2020 


\section{Abstract}

Video data include a significant amount of useful information that can be exploited by different organizations to gain more insights into running their business. The data are large and growing at a high rate because cameras, nowadays, are installed everywhere, gathering information all the time. Therefore, the data need large storage to be preserved and large computing power to be processed. Different technologies such as Apache Spark, Apache Storm, and Apache Hadoop have been widely used to perform big data processing on computer clusters. This thesis introduces general solutions and algorithms that can be used with different technologies, such as Apache Spark, Apache Storm, and Apache Hadoop, to improve the performance of processing big video data on computer clusters. However, the thesis focuses on using Apache Hadoop to provide an empirical evaluation of the proposed algorithms. Apache Hadoop is selected since it has been designed to work on commodity hardware. This thesis has been investigating different approaches to improve the performance of video processing on Hadoop clusters. These approaches are based on using the Hadoop MapReduce programming model to distribute the processing of big video data, and different sampling methods to avoid unnecessary computation while processing the video data. Change detection is used to detect the changes based on which the proposed algorithms sample the frames. The proposed algorithms can support video processing on faulty systems as well. The thesis also proposes three novel data placement policies to improve the performance of MapReduce-based video processing. 


\section{Acknowledgments}

I would like to express my gratitude to my Supervisors Prof. Shikharesh Majumdar and Prof. Anil Maheshwari for the continuous support of my PhD thesis. Also, I would like to thank Carleton University and the School of Computer Science for providing me with the required resources. 


\section{Table of Contents}

Abstract

Acknowledgments $\quad$ iv

Table of Contents $\quad$ v

List of Tables $\quad$ ix

List of Figures $\quad$ xi

List of Abbreviations $\quad$ xiv

List of Symbols $\quad$ xvii

1 Introduction 1

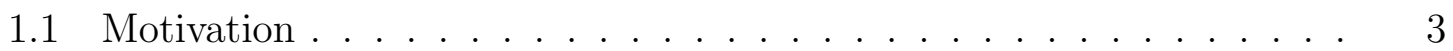

1.2 Proposed Performance Improvement Techniques . . . . . . . . . . . . 4

1.3 Thesis Contributions . . . . . . . . . . . . . . . . 7

1.3.1 Thesis Goals . . . . . . . . . . . . . . 7

1.3.2 Publications . . . . . . . . . . . . . . . . . . 9

1.4 Thesis Scope. . . . . . . . . . . . . . . . . 10

1.5 Thesis Outline....................... 10 
2.1 Change Detection $(\mathrm{CD}) \ldots \ldots \ldots \ldots 11$

2.1.1 CD Classification .................. . . 12

2.1.2 Using Gaussian Mixture Model . . . . . . . . . . . . . . 14

2.1.3 Change Detection Applications and Motivation . . . . . . . 15

2.2 Apache Hadoop . . . . . . . . . . . . . . . . . . . . . . 16

2.2.1 HDFS ............................... 17

2.2.2 Hadoop YARN . . . . . . . . . . . . . . . . . . . . . 17

2.2.3 Hadoop MapReduce . . . . . . . . . . . . . . . . . . . 18

2.3 Related Work . . . . . . . . . . . . . . . . 18

2.3.1 Storing video data in Hadoop clusters . . . . . . . . . . . . . . 19

2.3.2 MapReduce-based Change Detection . . . . . . . . . . . . . 20

2.3.3 Data Placement Policy . . . . . . . . . . . . . 21

2.4 Chapter Summary . . . . . . . . . . . . . . . . 25

3 The Video Splitting Approach 26

3.1 The Proposed Video Splitting Strategy . . . . . . . . . . . . . . 27

3.1.1 Video Splitting Modeling . . . . . . . . . . . . . . . . 28

3.1.2 Sampling Video Splitting (SVS) . . . . . . . . . . . . 31

3.1.3 MapReduce-based Change Detection . . . . . . . . . . . . 35

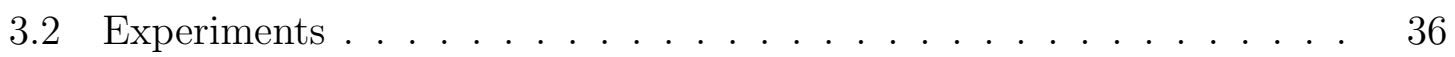

3.2.1 Accuracy Evaluation . . . . . . . . . . . . . . . 36

3.2.2 System Performance . . . . . . . . . . . . . . . 49

3.3 Chapter Summary . . . . . . . . . . . . . . 54

4 A Change Detection Approach Based on MapReduce and Sampling 56

4.1 Sampling-based Change Detection Approach . . . . . . . . . . 56

4.1.1 The MapReduce-Based Method . . . . . . . . . . . . 57 
4.1.2 The Traditional Change Detection Method . . . . . . . . . . . 58

4.1.3 The Proposed Sampling Method . . . . . . . . . . . . . . . 59

4.2 Experiments and Evaluation . . . . . . . . . . . . . 61

4.2.1 Accuracy Evaluation ................. 63

4.2.2 Performance Evaluation . . . . . . . . . . . . . . . . . . 67

4.3 Chapter Summary . . . . . . . . . . . . . . . 70

5 Change Detection on Fault-Tolerant Systems $\quad 72$

5.1 Overhead of MapReduce-Based Change Detection . . . . . . . . . . . 72

5.1.1 MapReduce-based CD Problem ... . . . . . . . . . 73

5.1.2 The Sampling of History Frames Based Approach . . . . . . . 74

5.1.3 The Sampling of Data Frames Based Approach . . . . . . . . 76

5.1.4 The Sampling of History and Data Frames Based Approach . 78

5.2 Experiments and Evaluation . . . . . . . . . . . . . 79

5.2 .1 Datasets . . . . . . . . . . . . . . . . . 79

5.2.2 Technologies and Cluster Setup . . . . . . . . . . . . . . . 80

5.2.3 MapReduce CD and Implementation details . . . . . . . . . 81

5.2.4 Evaluation of Execution Time . . . . . . . . . . . . . . . 82

5.2.5 Impact of the Video Segment Size . . . . . . . . . . . . . . . 86

5.2 .6 Impact of Input Data Size . . . . . . . . . . . . . . . . 88

5.2.7 Evaluation of Fault Tolerance . . . . . . . . . . . . . . . 90

5.3 Chapter Summary . . . . . . . . . . . . . . . . . . . . . 92

6 Data Placement Policy $\quad 94$

6.1 Modified Change Detection Approach . . . . . . . . . . . . 95

6.2 Proposed Data Placement Policies . . . . . . . . . . . . . . . . . 96

6.2.1 Challenge ...................... . . . 96

6.2.2 Content-wise Data Placement Policy . . . . . . . . . . . . . . 97 
6.2.3 Extended Content-wise Data Placement Policy . . . . . . . . . 101

6.2.4 Forward \& Backward Data Placement Policy . . . . . . . . . . 103

6.3 Experiments and Evaluation . . . . . . . . . . . . . . 105

6.3.1 Experimental Setup and Dataset . . . . . . . . . . 105

6.3.2 Experimental Results . . . . . . . . . . . . . . . . 107

6.4 Chapter Summary . . . . . . . . . . . . . . . . . . . . 112

7 Conclusions and Future Work 114

7.1 Conclusions . . . . . . . . . . . . . . . . . . . 114

7.2 Future Work . . . . . . . . . . . . . . . . . . . . . . 115

$\begin{array}{ll}\text { List of References } & 117\end{array}$

$\begin{array}{ll}\text { Appendix A Algorithms Details } & 127\end{array}$

A.1 SVSwS Algorithm . . . . . . . . . . . . . . . . 127

A.2 SVSwFW Algorithm . . . . . . . . . . . . . . . . 127

A.3 Sampling-based background model update . . . . . . . . . . . . . 128

A.4 CW-DPP Algorithm . . . . . . . . . . . . . 130

Appendix B Experimental Results 133

B.1 The Video Splitting Approach . . . . . . . . . . . . . . 133

B.2 Performance of the Proposed Sampling-based CD on a Fault Tolerant System . . . . . . . . . . . . . . . . . . . 134

B.3 The Experimental Results of Data Placement Policies . . . . . . . . . 135

$\begin{array}{ll}\text { Bibliography } & 163\end{array}$ 


\section{List of Tables}

3.1 Accuracy Evaluation Parameters. . . . . . . . . . . . . . 40

4.1 SdCD and SimpleCD execution time (seconds) comparison. . . . . . 68

6.1 Data placement policies summary _. . . . . . . . . . . . 113

B.1 Accuracy of NVSwO for the PETS2006 dataset. . . . . . . . . . 136

B.2 Accuracy of SVSwS for the PETS2006 dataset (different history sizes). 137

B.3 Accuracy of SVSwS for the PETS2006 dataset (different strides). . . 138

B.4 Accuracy of SVSwFW for the PETS2006 dataset. . . . . . . . . . 139

B.5 Accuracy of NVSwO for the Skating dataset. . . . . . . . . . 140

B.6 Accuracy of SVSwS for the skating dataset (different history sizes). . 141

B.7 Accuracy of SVSwS for the skating dataset (different strides). . . . 142

B.8 Accuracy of SVSwFW for the skating dataset. . . . . . . . . 143

B.9 Accuracy of NVSwO for the Highway dataset. . . . . . . . . . . 144

B.10 Accuracy of SVSwS for the highway dataset (different history sizes). . 145

B.11 Accuracy of SVSwS for the highway dataset (different strides). . . . 146

B.12 Accuracy of SVSwFW for the highway dataset. . . . . . . . 147

B.13 Increasing the degree of parallelism (weekday). . . . . . . . . 148

B.14 Increasing the degree of parallelism (weekend). . . . . . . . . . . 149

B.15 Segment size and execution time (weekday). . . . . . . . . . 149

B.16 Segment size and execution time improvement (weekday). . . . . . 150

B.17 Segment size and execution time (weekend). . . . . . . . 150 
B.18 Segment size and execution time improvement (weekend). . . . . . 150

B.19 No. of input files and execution time (weekday). . . . . . . . . 150

B.20 No. of input files and execution time (weekend). . . . . . . . . 151

B.21 One node failure recovery and execution time. . . . . . . . . 151

B.22 One node failure recovery and execution time deterioration. . . . . . . 151

B.23 One-node failure recovery and point of failure (PoF) . . . . . . . . 151

B.24 Two-node failure recovery and execution time. . . . . . . . . 152

B.25 Increasing data size effect (weekday). . . . . . . . . . . . . . 152

B.26 Increasing data size effect (weekend). . . . . . . . . . . . . 152

B.27 The effect of the no. of replicas. . . . . . . . . . . . 152

B.28 One-node failure recovery. . . . . . . . . . . . . . 153

B.29 Two-node failure recovery. . . . . . . . . . . . . . . 153 


\section{List of Figures}

3.1 Naive Video Splitting with Overlapping (NVSwO) model. . . . . . . . 30

3.2 Sampling Video Splitting with Fibonacci Window (SVSwFW). . . . . 33

3.3 Advantage of using Fibonacci Window. . . . . . . . . . . . . . . . 34

3.4 PETS2006 dataset. . . . . . . . . . . . . . . . . . . 38

3.5 Skating dataset. . . . . . . . . . . . . . . . . . . . 38

3.6 Highway dataset. . . . . . . . . . . . . . . . . . . . . 39

3.7 NVSwO $($ PETS2006) . . . . . . . . . . . . . . . . 41

3.8 SVSwS $($ PETS2006). . . . . . . . . . . . . . . 41

3.9 SVSwS with different strides (Modified PETS2006) . . . . . . . . . . 42

3.10 SVSwFW (Modified PETS2006). . . . . . . . . . . . 43

3.11 Comparing NVSwO, SVSwS and SVSwFW (Modified PETS2006). . . 43

3.12 NVSwO (Skating). . . . . . . . . . . . . . . . . 44

3.13 SVSwS (skating). . . . . . . . . . . . . . . . . 45

3.14 Using SVSwS with different strides (Skating). . . . . . . . . . . 45

3.15 Using SVSwFW (skating scenario). . . . . . . . . . . 46

3.16 Comparing NVSwO, SVSwS and SVSFW (skating). . . . . . . . 46

3.17 NVSwO (Highway). . . . . . . . . . . . . . . . . . . . 47

3.18 SVSwS (Highway). . . . . . . . . . . . . . . . . 48

3.19 Using SVSwS with different strides (Highway). . . . . . . . . . . 48

3.20 SVSwFW (Highway). . . . . . . . . . . . . . . . . . . 49 
3.21 Comparing NVSwO, SVSwS and SVSFW (Highway). . . . . . . . . 49

3.22 The effect of SVSwFW and NVSwO on the execution time (PETS2006 dataset). . . . . . . . . . . . . . . . . 52

3.23 The effect of SVSwFW and NVSwO on the speedup (PETS2006 dataset). 52

3.24 The effect of SVSwFW and NVSwO on the efficiency (PETS2006 dataset). . . . . . . . . . . . . . . . 53

3.25 The effect of SVSwFW and NVSwO on the execution time (skating

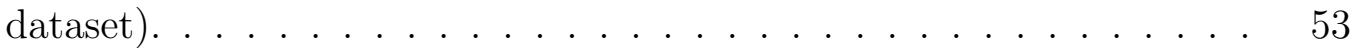

3.26 The effect of SVSwFW and NVSwO on the speedup (skating dataset). 54

3.27 The effect of SVSwFW and NVSwO on the efficiency (skating dataset). 54

4.1 Displaying AoC in frame (a) as a binary mask frame (b) . . . . . . . . 62

4.2 Accuracy test for the skating dataset. . . . . . . . . . . . 65

4.3 Example of how the threshold values ( $\mathrm{Tl}$ and $\mathrm{Th}$ ) decide sampling. . 66

4.4 Execution time as the number of nodes increases. . . . . . . . . . . . 69

4.5 The execution time improvement achieved by SdCD. . . . . . . . . . 70

4.6 Speedup. . . . . . . . . . . . . . . . . . . 71

5.1 Data frames sampling technique. . . . . . . . . . . . . . 78

5.2 Degree of parallelism and execution time (weekday) . . . . . . . . . . 82

5.3 Degree of parallelism and execution time improvement (weekday). . . 83

5.4 Degree of parallelism and speedup (weekday). . . . . . . . . . 83

5.5 Degree of parallelism and efficiency (weekday). . . . . . . . . . . . 84

5.6 Degree of parallelism and execution time (weekend) . . . . . . . . 85

5.7 Degree of parallelism and execution time improvement (weekend). . . 85

5.8 Degree of parallelism and speedup (weekend). . . . . . . . . . . 86

5.9 Degree of parallelism and efficiency (weekend) . . . . . . . . . . 86

5.10 Segment size and execution time (weekday) . . . . . . . . . . . 87

5.11 Segment size and execution time improvement (weekday). . . . . . . 87 
5.12 Segment size and execution time (weekend). . . . . . . . . . . . 88

5.13 Segment size and execution time improvement (weekend) . . . . . . 88

5.14 No. of video files and execution time (weekday) . . . . . . . . . . 89

5.15 No. of video files and execution time (weekend). . . . . . . . . . . 89

5.16 One-node failure recovery and execution time. . . . . . . . . . . 90

5.17 One node failure recovery and execution time deterioration. . . . . . . 91

5.18 One-node failure recovery and point of failure $(\mathrm{PoF}) . \quad \ldots . . . . .991$

5.19 Two-node failure recovery and execution time. . . . . . . . . . . . . . 92

6.1 Example of Data blocks placement. . . . . . . . . . . . . . . 98

6.2 An example to demonstrate how CW-DPP works. . . . . . . . . . . 102

6.3 Increasing data size and execution time (weekday) . . . . . . . . . . 107

6.4 Increasing data size and locality (weekday). . . . . . . . . . . . . 108

6.5 Increasing data size and execution time (weekend) . . . . . . . . . . . 108

6.6 Increasing data size and locality (weekend) . . . . . . . . . . . . . 109

6.7 The effect of the no. of replicas on execution time. . . . . . . . . . . 109

6.8 The effect of the no. of replicas on the Locality. . . . . . . . . . . . . 110

6.9 Effect of one-node failure on execution time. . . . . . . . . . . . . . . 110

6.10 Effect of one-node failure on the locality. . . . . . . . . . . . . . . 111

6.11 Effect of two-node failure on execution time. . . . . . . . . . . . . . . 111

6.12 Effect of two-node failure on the locality. . . . . . . . . . . . . . . 112 


\title{
List of Abbreviations
}

\author{
AoC \\ Amount of Change \\ BS \\ Background Subtraction \\ CD \\ Change Detection \\ CW-DPP \\ Content-Wise Data Placement Policy \\ Default-DPP \\ Hadoop Default Data Placement Policy \\ DPP \\ Data Placement Policy \\ DSW \\ Data Sampling Window \\ ECW-DPP \\ Extended Content-Wise Data Placement Policy \\ F\&B-DPP Forward \& Backward Data Placement Policy \\ FibWin Fibonacci Window \\ FN \\ False Negatives \\ FP \\ False Positives \\ FPR \\ False Positive Rate \\ GMM \\ Gaussian Mixture Model




\begin{tabular}{|c|c|}
\hline GOP & Group of Pictures \\
\hline HDFS & Hadoop Distributed File System \\
\hline HSW & History Sampling Window \\
\hline NED-DPP & Naive Even Distribution Data Placement Policy \\
\hline Normal-MR & Normal MapReduce Application \\
\hline NU-MR & Non Uniform MapReduce Application \\
\hline NVS & Naive Video Segmentation \\
\hline $\mathrm{NVSwO}$ & Naive Video Segmentation with Overlapping \\
\hline PoF & Point of Failure \\
\hline RoI & Region of Interest \\
\hline $\mathrm{SdCD}$ & Sampling Data Frames based Change Detection \\
\hline $\mathrm{ShCD}$ & Sampling History Frames based Change Detection \\
\hline ShdCD & Sampling History \& Data Frames based Change Detection \\
\hline SimpleCD & Simple Change Detection \\
\hline SVS & Sampling Video Segmentation \\
\hline SVSwFW & Sampling Video Segmentation with Fibonacci Window \\
\hline SVSwS & Sampling Video Segmentation with Stride \\
\hline $\mathrm{TN}$ & True Negatives \\
\hline $\mathrm{TP}$ & True Positives \\
\hline
\end{tabular}


TPR

VS

YARN
True Positive Rate

Video Segmentation

Yet Another Resource Negotiator 


\section{List of Symbols}

$\begin{array}{ll}\overline{A o C} & \text { Average amount of change for a video segment } \\ b_{j}^{0} & \text { First replica for video segment } j \\ b_{j}^{1} & \text { Second replica for video segment } j \\ C & \text { Useful computation time } \\ d & \text { Data frames size } \\ F & \text { Big video file } \\ f_{b g}^{0} & \text { Initial background model } \\ f_{b g}^{t} & \text { Background model at time } t \\ f_{b m}^{t} & \text { Binary mask frame at time } t \\ f_{d}^{t} & \text { Data frame at time } t \\ f_{h}^{i} & \text { Gth historyframe } \\ g & \text { Group size } \\ h & \text { History frames size } \\ \mathcal{N} & \text { Set of datanodes }\end{array}$


O

$p$

\section{$r$}

st

$T_{h}$

$T_{l}$

$U_{k}$

V

$\mathcal{V}$

$V^{j}$

$V_{d}^{j}$

$V_{b m}^{j}$

$V_{h}^{j}$

$V_{h^{\prime}}^{j}$

$w_{d}$

$w_{h}$

$\alpha$

$\alpha^{\prime}$

$\tau$
Overhead of processing overlapping frames twice

Degree of parallelism or number of segment

Round number

Stride number

High threshold value

Low threshold value

Utilization list of group $k$

Set of all video segments

Set of related video segments

Video segment $j$

Set of all data frames of video segment $j$

Set of all binary mask frames

Set of all history frames of video segment $j$

Set of sample history frames of video segment $j$

Size of DSW

Size of HSW

Overhead of processing overlapping frames twice

Overhead of processing the sample overlapping frames twice

The saved execution time because of sampling 


\section{Chapter 1}

\section{Introduction}

The importance of video data is reflected in the large amount of useful information held in such data, so the field of video processing attracts the attention of many researchers. The challenge is that video data are large in size, so they require significant computing power to be processed in a timely manner. This challenge can be addressed by devising more efficient algorithms or by exploiting the parallel and distributed computing. The proposed approaches can be utilized to improve the performance of different video processing applications, such as detecting and tracking mobile objects. They also can improve the performance of different video surveillance systems that identify and analyze human activities, such as detecting incidents on highways and tracking suspects in public space. The approaches can also improve the performance of detecting the Region of Interest (RoI) that plays a pivotal role in reducing the computation in a variety of video processing applications.

Change Detection (CD) or Background Subtraction (BS) is one of the most commonly used steps in many video surveillance applications. According to [1], reducing the execution time of BS algorithms can be categorized under three approaches. The first one is to optimize the algorithm so that the amount of computation can be minimized. In [2], the author improved the Gaussian Mixture Model (GMM) proposed in [3] by changing the number of components for each pixel based on the scene. As a 
result, the proposed algorithm leads to lower execution time.

The second approach is based on sampling to reduce computation time. The authors in [1] proposed a foreground probability map method that utilizes some foreground properties. The foreground probability map is a model that gives a high probability for foreground pixels based on temporal, spatial, and frequency properties, and it is adapted based on the previous foreground detection results. The paper [4] used an experiential sampling technique to improve the speed of GMM. The idea of the experiential sampling is to reduce the computation by focusing on the most relevant pixels changing through the previous frames. In [5], the authors proposed a BS algorithm that uses a two-level sampling technique to reduce computation time. The idea is that instead of involving the pixels of the entire input image, the image is divided into blocks that will be sampled. After the block level sampling, another sampling at the pixel level is performed to reduce the computational load even more. In [6], using a space sampling method to increase the processing speed of GMM was proposed. The idea of space sampling is to reduce the resolution of the input image before applying GMM to the low-resolution image. The output binary mask will be zoomed back to the original input image size. Finally, the zoomed mask will be used to obtain the foreground objects.

The third approach is based on using parallel computing under different architectures. On multi-core architecture, the authors of [7] proposed a parallel implementation for GMM using the OpenMP framework. On GPU architecture, there are many implementations to parallelize BS using different optimization techniques (such as memory coalescing, data transfer, kernel overlapping, divergent branch elimination, and efficient register usage) [8-10]. The research described in [11] proposed a parallel implementation of the CodeBook model on GPU to achieve BS. On distributed memory systems, the authors of [12] proposed a parallel algorithm of the classical Gaussian model to support real-time video applications depending on CD. 
In the previous works, the goal of using the three approaches is to reduce the execution time of CD to satisfy the real-time performance for some video processing applications. Another reason for reducing the execution time of CD is to process large video data in a timely manner. This thesis uses Hadoop technology to store and process big video data in parallel. Apache Hadoop is an open-source platform devised to support distributed storage and processing of big data $[13,14]$. The Hadoop platform encompasses two main components: the Hadoop Distributed File System (HDFS) and the MapReduce programming model. The role of HDFS is to divide large data files into blocks before storing the blocks across a Hadoop cluster. On the other hand, the purpose of the MapReduce programming model is to process the data stored in HDFS in parallel.

\subsection{Motivation}

The authors of [15] have introduced a study to review big data processing on cloud computing and to highlight some open research issues. They indicate that the heterogeneity of unstructured data, such as video, and the way how to store and retrieve massive amounts of data are open research issues that need to be solved. To process video data on Hadoop clusters, a video file should be first stored in HDFS. HDFS divides the video files into blocks and stores the blocks across the cluster nodes $[13,14]$. Storing video files is more challenging than storing text files. This is because the video frames are stored within a container that includes the video data as well as other information such as the video coding format (video codec) used. Storing a video file directly in HDFS will destroy the container format and the correlation between the frames if the file size is larger than the HDFS block. Consequently, the video file needs to be divided into independent video segments with sizes smaller than the HDFS block before being uploaded to HDFS. 
Different works in the literature have been trying to perform big video data processing on Hadoop clusters by preprocessing the video data before uploading the data to HDFS and extending some MapReduce classes and Interfaces. Change detection has been utilized in many applications to perform big data processing on Hadoop clusters [16-20]; however, these works have focused merely on using the parallel model of MapReduce. The motivation of this thesis is to propose a number of techniques for improving the performance of MapReduce-based CD on Hadoop clusters. Furthermore, a novel data placement policy is also devised to improve the performance of the proposed MapReduce-based CD jobs.

\subsection{Proposed Performance Improvement Tech- niques}

The main goal of this thesis is to improve the performance of video processing applications based on change detection methods on Hadoop clusters. Change detection is a preliminary step used in video surveillance applications to specify RoI to restrict the computation on the useful part of video data. It also can be used in different applications such as mobile object tracking, people counting, as well as crowd and traffic monitoring [21]. Gaussian Mixture Model [2] is the change detection algorithm used in this thesis to demonstrate the effect of the different approaches and algorithms to improve the performance on Hadoop clusters. This thesis improves the performance of change detection on Hadoop clusters by exploiting the MapReduce programming model to achieve parallel processing and using different sampling techniques to avoid unnecessary computations as follows.

The first approach is to improve the performance by devising techniques for partitioning video files before uploading the data to HDFS. The change detection algorithm 
(GMM) requires some history frames to estimate the initial background model. In the serial run, there is only one set of history frames (training set) at the beginning of the video sequence. To process the video sequence in parallel, it should be partitioned into segments and distributed across the nodes. In this case, each segment requires a training set to generate the initial background model. The training set of each segment is overlapped from the previous segment. There are two categories of video processing applications based on the correlation between the video frames [16]: single-frame oriented, and frame-series oriented. In the former one, each video frame can be processed independently. An object detection application is an example of this category. In the latter category, a group of frames needs to be considered in addition to the frame meant to be processed. A CD algorithm is an example of this category. Dividing a video file to support single-frame oriented applications on Hadoop clusters is straightforward. However, dividing the file to support frame-series oriented applications needs overlapping some frames between every two consecutive segments. The more the number of overlapping frames and video segments, the more processing overhead is encountered. This is because the overlapping frames will be processed twice. To reduce the overhead, a novel video splitting approach that can support frame-series oriented video applications on Hadoop clusters is proposed.

The second approach introduced in this thesis to improve the performance of the change detection algorithm is to use sampling to avoid unnecessary computations while processing the frames of a particular video segment. Fortunately, in the realm of video processing, it is not necessary to process the entire video sequence frame by frame, but instead, the frames that hold useful information can be processed. A sampling-based method is devised in this thesis to avoid unnecessary computation. Therefore, more video data can be processed in a timely manner.

Third, the thesis also includes an empirical study to demonstrate the effect of different sampling techniques on the performance of MapReduce-based video processing 
on Hadoop clusters. When change detection is used to process video segments on a Hadoop cluster, it considers two types of frames: history frames and data frames. Three sampling approaches are proposed and investigated to give more insight into the effect of sampling history frames, data frames, or both history and data frames. The proposed sampling approaches have different effects on the running MapReducebased video jobs in case of fault occurrence. The thesis gives more insight into the performance of the running applications if there is a node failure. Although the sampling-based approaches improve the execution time, they result in workload imbalance and data migration overhead. To solve these problems, the data placement policy of the Hadoop distributed file system needs to be extended (Chapter 6).

The last contribution of this thesis research is to introduce a Data Placement Policy to store the video data in Hadoop clusters in such a way that the performance of the running video processing jobs can be improved. HDFS stores data blocks according to Hadoop's default data placement policy [13], which is designed to guarantee reliability and availability. This policy distributes the blocks of a single file evenly across cluster nodes. In this case, a MapReduce job that processes the single file performs well under homogeneous Hadoop clusters. This is because the map tasks will access the data locally. When the default data placement policy stores multiple files, it results in storage load imbalance because of the randomness of placing the files. As a consequence, a MapReduce job intended to process the files inevitably experiences access load imbalance. This is because some nodes process the data locally while the others need to transfer remote data via the network, and then process the data locally. Transferring large amounts of data across the network leads to degrading system performance and increasing resource consumptions. Consuming resources in Cloud platforms would come at a high cost, especially in large-scale Hadoop clusters. The desired data placement policy should minimize data transfer around the network (i.e. minimizing access load imbalance), especially in Cloud-based systems 
since transferring large amounts of data comes at a high cost. Using the proposed sampling techniques results in different execution time even if the video segments have the same size. This is because the sampling techniques depend on the content of the data to make the sampling decision. For example, suppose there are two video segments $\mathrm{S} 1$ and $\mathrm{S} 2$, and there is a lot of changes in segment $\mathrm{S} 1$ and fewer changes in $\mathrm{S} 2$. This means that $\mathrm{S} 1$ will not use sampling, but $\mathrm{S} 2$ will use sampling to update the background model. Therefore, the execution time of S1 will be larger than S2. This disparity in execution time occurs even if the cluster is homogeneous, and it causes access load imbalance since the node that finished processing the local data will transfer remote data before processing the data locally. This gives the motivation to devise a novel data placement policy to minimize the access load imbalance.

\subsection{Thesis Contributions}

\subsubsection{Thesis Goals}

The contributions of this thesis can be summarized as follows:

- Two video splitting techniques: Since big video data require to be divided so that they can be stored and processed in a distributed manner, this thesis proposes two sampling-based techniques to partition large video file. The goal of using sampling is to minimize the overhead resulted in by overlapping some frames between consecutive segments. The thesis includes insights into:

- The effect of the proposed techniques on the accuracy: Running change detection requires a training data set for each video segment. Since each video segment uses a sample training set, estimating the background model 
for each segment will affect the accuracy. The experimental results demonstrate how the proposed techniques use fewer frames to estimate the background model without significantly affecting the accuracy of the model.

- The performance of processing the segmented video data: The goal of the proposed techniques to segment big video data is to minimize the overhead resulting from overlapping the frames of the training sets. This part of the contribution is to show the performance improvement gained by processing the video data partitioned using the proposed segmentation techniques.

- A change detection approach based on MapReduce and sampling: A novel change detection approach based on using the MapReduce programming model to distribute storing and processing large video data, and a sampling method to avoid processing unnecessary computation dynamically based on the content of the processed data. This approach is called Sampling Data Change Detection (SdCD). This part includes insights into:

- The accuracy of SdCD: The accuracy evaluation compares the proposed change detection approach that tries dynamically to update the background model based on the amount of changes in the scene with the traditional approach used in the literature that only uses MapReduce without sampling. The experimental results show that the proposed SdCD does not significantly affect the accuracy of change detection.

- The performance of SdCD: This section introduces the performance improvement gained by using sampling MapReduce CD in comparison with CD that uses the MapReduce programming model.

- Insights into the impact of using sampling with the occurrence of

faults: Three algorithms that sample the training set, the video data, or both 
have been proposed to investigate the effect of sampling on the performance of MapReduce CD when faults occur on the system.

- Three custom Data Placement Policies (DPPs): The data placement policies can distribute the video data in such a way that they minimize the overhead of transferring the data around the network. The data placement policy can play an important role in improving not only the performance of the running application but also the entire system performance by improving the locality of references and balancing the workload in a Hadoop cluster. The experimental results give more insight into how the proposed data placement policies improve the performance on Hadoop clusters.

\subsubsection{Publications}

\section{Published Papers:}

- E. SaatiAlsoruji and S. Majumdar, "A Video Segmentation Strategy for Video Processing Applications on Hadoop Clusters," in IEEE SmartWorld, Ubiquitous Intelligence \& Computing, Advanced \& Trusted Computing, Scalable Computing \& Communications, Cloud \& Big Data Computing, Internet of People and Smart City Innovation (SmartWorld/SCALCOM/UIC/ATC/CBDCom/IOP/SCI), (Guangzhou, China), 2018.

- E. SaatiAlsoruji, " A Change Detection Approach for Processing Outdoor Scenes on Hadoop Clusters," in International Conference on Future Internet of Things and Cloud (FiCloud 2019), (Istanbul, Turkey), 2019.

- E. SaatiAlsoruji, "Improving the Performance of MapReduce-based Change Detection Using Sampling," in IEEE Conference on Big Data and Analytics (ICBDA 2019), (Pulau Pinang, Malaysia), 2019. 


\subsection{Thesis Scope}

Hadoop technology has been introduced in the literature to store and process large video data. Hadoop can be used to store large multimedia files in a distributed manner in a cluster, and these data can be retrieved for streaming. However, the scope of this thesis concerns the storage of large video data with a specific focus on video data processing for surveillance purposes. For example, assume that there is a surveillance system to monitor the activities of people in a shopping center. In this case, the size of the recorded video data will be very large, and thereby the storage capability of one device will not be sufficient. This problem can be solved by using multiple devices to store the recorded data.

HDFS provides distributed storage to store large video data. The video data are encoded using the cameras and distributed across the datanodes that facilitate processing the stored video data using the MapReduce programming model. To process the stored data, the FFmpeg library [22] is used to decode the different stored video file format.

\subsection{Thesis Outline}

The remainder of this thesis proposal is structured as follows: Chapter 2 reviews some background information as well as related work. Chapter 3 describes the proposed video splitting strategy. Chapter 4 introduces the sampling MapReduce CD approach. Chapter 5 discusses the effect of sampling on MapReduce-based change detection in case of fault occurrence. Chapter 6 describes a data placement policy that improves the performance of the system running MapReduce CD based on sampling. Chapter 7 concludes the thesis and introduces the future work. 


\section{Chapter 2}

\section{Background and Related Work}

The main goal of this thesis is to improve the performance of change detection used to process video data that have large size and need significant computing power for processing. Apache Hadoop is a general-purpose framework that can be utilized to support processing large video data. This chapter gives an overview of the Hadoop framework and some related work of using Hadoop to implement different video processing applications.

\subsection{Change Detection (CD)}

A variety of video surveillance applications require to model the background in order to detect the moving objects or the changes in different video scenes. The background can be obtained by having the scene without moving objects. The background image can be subtracted from the current video frames to detect the foreground moving objects (changes) in the scene. However, unfortunately, in many cases, it is not possible to acquire the background image. Furthermore, even if we have the background, it could be changing because of adding/removing some objects to/from the scene, or changing the illumination for instance. Hence, the discipline of modeling the background interests many researchers. In literature, the topic of background modeling is 
also referred to as background subtraction or change detection.

\subsubsection{Classification}

There are many background modeling methods proposed in the literature. Boumans et al. [23] introduce a taxonomy that classifies the background modeling approaches from 4 different perspectives: the common computational method (methodology), data abstraction, number of simultaneously considered video frames (recursivity), and selected whole/subsets video information (selectivity). The following subsections will explain the four classifications.

\subsubsection{Methodology-based Classification}

In the first category, there are many methods that depend on Temporal Statistics (TS), including mean, median, or mode. The computation is on the pixel-level for the whole video sequence such as [24-29] or for randomly selected frames, such as $[30,31]$. There also are several popular background modeling approaches, such as $[2,3,32]$. The main idea of these approaches is to commence with an initial background model and keep updating the model to correct the initial errors. The methods of the second category, Subsequence of Stable Intensity (SSI), consist of two phases, and they depend on the assumption that the values of the background pixels are stable over a long period [33-35]. Firstly, for each pixel or image block, various subsequences having similar intensity values are selected. Secondly, the best candidate subsequence to represent the background will be selected based on some criteria. The methods under the third category, Iterative Model Completion (IMC), consist of two phases, and the goal is to iteratively build the background model [36-38]. In the first phase, a static image region is selected as a reference background. After that, the background model is iteratively built according to spatial consistency criteria. In [39-41], the approaches model the background as an Optimal Labeling (OL) problem. The label computed 
for each image region gives the best frames in which the background information is included. The background model is formed from the background information of the frames selected by minimizing the cost function that considers the spatio-temporal information. The approaches in [42-44] are based on initializing the background model by using the missing data to reconstruct the background. Missing data occur because of the existence of moving foreground objects. Many approaches, such as [45-47], are based on Neural Networks (NN), where the background model can be automatically learned using video data in supervised and unsupervised ways. Deep learning has recently been used to improve the model accuracy [48-50].

\subsubsection{Data Abstraction-based Classification}

Under this classification, there are three categories. The first category is known as pixel-level methods that use the temporal consistency of the pixel intensity over time observations $[3,30,51,52]$. These approaches hardly tackle situations such as sudden illumination changes since the spatial relations between pixels are not considered. According to the second category (region-level methods), the approaches in $[34,38,53]$ use both spatial and temporal information. According to these approaches, the images are divided into regions based on the inter-pixel relations, so they are computationally intensive in comparison with the previous class. In the third category, hybrid methods, both pixel-level, and region-level are combined [54,55]. These methods provide a trade-off between efficiency and accuracy.

\subsubsection{Recursivity-based Methods}

Recursive methods such as $[3,29,32,35,36,38,40,56,57]$ analyze an on-line new sequence frame and use it for estimating and updating the background model in an iterative manner. The updated background model considers slow-moving objects and stationary foreground ones. In non-recursive methods [24, 25, 30, 31, 33, 34, 41], an 
off-line background model is estimated. The disadvantage of these methods is that they are demanding of memory resources.

\subsubsection{Selectivity-based Methods}

In this classification, there are two categories. In the first category, the methods construct the background model for each pixel using the pixel intensities over the

whole time [3,29-32]. These methods give information about the background and the foreground resulted in by the moving objects covering the pixels along the sequence. In the second category, the methods separate the background from the foreground over a selected subset of video information. Most of the existing approaches fall under this category.

\subsubsection{Using Gaussian Mixture Model}

In this thesis, the OpenCV implementation for GMM is used [2]. The idea is to model the value of each pixel as a mixture of Gaussians. The scene could be changing over time because of daytime, weather conditions, abrupt illumination changes, or occluding the background with foreground static objects. Therefore, GMM is used to model each pixel in the scene to reflect the previously mentioned cases since the traditional Gaussian method fails in these situations. Pixels are deemed foreground if they do not match any background distribution. A training set considered from history is used to estimate the initial background model. The background model can be subtracted from the current frame to detect the changes incurred by moving foreground objects. The subtraction in this context means that each pixel value in the background model is to be subtracted from the value of the counterpart pixel value in the current frame, and the square of the distance between the two values is calculated. After that, if the square difference is larger than a predefined threshold (parameter), the pixel belongs to the foreground object (signifying change detection); 
otherwise, it belongs to the background. The algorithm takes three parameters: history, varThreshold, and detectShadows. The history is the parameter to affect updating the background model and its default value is 500 frames. The varThreshold is the parameter to decide whether the pixel belongs to the model, and it does not affect updating the model. The detectShadows parameter is to decide whether to consider the moving objects' shadows or not, and it is true by default. In sum, GMM generates the initial background model and keeps updating the model according to the previously described parameters depending on the scene. For example, if the scene is crowded, more history frames are required to generate and update the background model in comparison with the same scene with less crowds.

\subsubsection{Change Detection Applications and Motivation}

Change detection has been attracting the attention of many researchers over the past decades. As discussed in Section 2.1.2, a wide range of methods have been proposed to improve its performance. The answer to the question that why CD attracts the attention of researchers is that it plays an important role in many computer vision applications. A survey conducted in [58] shows different applications that depend on CD as the first step.

- Using video surveillance to identify human activities: CD is used in different traffic surveillance applications such as tracking vehicles [59], counting the number of vehicles [60], or congestion detection [61]. It can also be used to detect and track customers in stores $[62,63]$.

- Using video processing for observing animals and insects behaviors: Videobased systems were used to analyze the interactions of honeybees [64] and mice $[65,66]$ inside their groups. Video systems have also been used to analyze the relationship between birds and the environments to acquire information about 
the effect of climate change on the ecosystem $[67,68]$. In $[69,70]$, the authors have developed computer vision algorithms to record the location and movement of some endangered species for later review.

- Observing Natural Environments: The goal of this type of applications is to detect foreign objects in natural environments for protecting biodiversity, such as detecting the floating objects on the sea surface [71].

- Analyzing human activities: CD can be used to analyze the performance of athletes, such as detecting and analyzing rowing motion in video sequences [72].

- Vision and gesture recognition: In this type of applications, detecting, tracking, and recognizing hand gestures are required in a variety of applications involving human-computer interface, behavior studies, sign interpretation, robotics, and games [58].

- Other applications: CD can also be used in miscellaneous applications such as baggage detection [73] and fire detection [74].

As shown above, CD can be used in a wide range of applications. This provides the motivation for the thesis to improve its performance in the Hadoop environment. By improving CD to perform different video processing applications, many video-based applications can consequently analyze big video data more efficiently on Hadoop clusters, especially on clusters that are hosted on a Cloud.

\subsection{Apache Hadoop}

Apache Hadoop is an open framework to facilitate the storing and processing of big data. It includes 3 core components [13,14]: Hadoop Distributed File System, Hadoop YARN, and Hadoop MapReduce. 


\subsubsection{HDFS}

HDFS is designed to deal with very large files that cannot be accommodated in a single disk [14]. This can be achieved by distributing a single large file on different nodes so that developers can parallelize not only the processing but also accessing the different parts of the file overcoming the problem of single-disk access latency. HDFS involves two types of nodes to manage the stored files: a namenode and multiple datanodes. To store a large file on a Hadoop cluster, HDFS divides the file into evenly sized blocks distributed across a number of datanodes. Each block coexists with a number of replicas distributed across the cluster according to a configurable parameter (dfs.replication). Storing the location information of each block belonging to a particular file is the responsibility of the namenode that also manages the file system, divides the files into blocks, deploys the blocks over the cluster, and regulates accessing of the files. When a MapReduce job is running, it sends a request to the namenode to specify the datanodes on which the required data are stored. This is important for processing the data locally.

\subsubsection{Hadoop YARN}

YARN, which stands for Yet Another Resource Negotiator, is the resource manager in Hadoop clusters. It provisions MapReduce applications (discussed in the following subsection) with the cluster resources. The services offered by YARN are introduced through two types of daemons: a single resource manager running on a node called master and multiple node managers running on nodes called slaves. There is one resource manager on each cluster, and it is responsible for allocating the resources to the running applications based on their resource requirements. YARN accepts a job and tries to find a suitable container to run an application master for that job. A container is a process with constrained resources (CPU, memory) in which a 
map/reduce task or application master is running. The function of the application master (one per an application) is to negotiate with the scheduler component of the resource manager to acquire the required containers for running the map and reduce tasks that belong to the same application. A node manager is a daemon that exists on each slave node in the Hadoop cluster to provide its resources (CPUs, memory, disks, etc.) to the running MapReduce applications in the cluster. Its responsibility is to create containers, monitor the usage of the local resources, and report back the resource usage statistics to the resource manager.

\subsubsection{Hadoop MapReduce}

MapReduce is a distributed programming model [13], which embodies the divide and conquer strategy to tackle big data stored in Hadoop clusters. MapReduce has two phases that are used to distribute the computation: the map phase and the reduce phase. The data stored in HDFS is split into chunks called InputSplits so that each InputSplit can be processed by one map task. The map tasks read the InputSplits as key-value pairs to process the data in parallel. The output of the map tasks is sent as intermediate key-value pairs to reduce tasks. The reduce tasks process the intermediate output of the map tasks as input and then write the final output back to HDFS.

\subsection{Related Work}

HDFS divides an uploaded file into blocks and places them in different nodes in a Hadoop cluster according to the default block placement policy $[13,14]$. After that, to run a MapReduce application on the stored data, Hadoop divides the input data

into splits called InputSplits and assigns one map task for each InputSplit. Since video data are encoded, uploading a video file directly will damage the container 
format when the video file is divided into blocks. Furthermore, MapReduce does not support video processing by default; however, since Hadoop is a general-purpose framework, some MapReduce classes are required to be extended. This thesis focuses on improving the performance of change detection through three approaches: (1) the strategy used to segment big video data, (2) the MapReduce approach used to process the video data, and (3) the distribution of the video data on Hadoop clusters by using the data placement policy. The following subsections will introduce the related work for each of the previously mentioned approaches.

\subsubsection{Storing video data in Hadoop clusters}

To handle the problem of distributing the video data across a Hadoop cluster, there have been 4 main strategies in the literature that are briefly described as follows. The first, advocates the extracting of video frames and then converting them into sequence files. After that, the sequence files are uploaded to HDFS as in $[75,76]$. The disadvantage of this technique is that storing the video frames as sequence files takes more space than storing them as video files. The second strategy is to divide a large video file into a group of pictures (GOPs). According to this strategy, the video GOP elements can be distributed across the cluster nodes. GOP is a set of video frames encoded together. In [77], the authors used this strategy in a parallel face tracking application on Hadoop clusters. The disadvantage of this strategy is that it does not support all video formats [17]. In MPEG-2 format, for instance, there are two types of GOP. In the first type, to decode the frames of one GOP, no other frames are required from other GOPs. The solution in [77] supports this type of video files since each InputSplit represents one GOP. In the second type, decoding the frames of a GOP requires some frames from other GOPS; therefore, this type of decoding is not supported by the proposed solution.

The third strategy is to assign each video to a single map task In this strategy, 
all the video file blocks need to be placed in the same node so that one map task will process one video file, and different video files can be processed by different nodes [17]. The drawback of this strategy is that when a few large video files are to be processed, the degree of parallelism will be confined to the number of the video files. The fourth strategy is to divide one video file into independent video segments. According to this video splitting strategy (VS), a video file is divided into many smaller video files called segments so that each segment preserves the container format required to decode its frames. The size of each video segment should be smaller than the size of the HDFS block to prevent dividing the segment into blocks. Some works such as $[19,36,78,79]$ focus on using this strategy. The advantage of using this strategy is that it allows us to divide a large video file into segments in such a way that each segment can be processed on a different node independently in parallel.

\subsubsection{MapReduce-based Change Detection}

In the literature, some researchers used CD (or BS) in their video processing applications that exploit Hadoop to speed up the computation. The authors of [16] propose an approach to speed up video processing on Hadoop clusters. They used background modeling to detect moving objects and track them. In [17], the authors have proposed a metadata extraction and metadata correction system implemented using Hadoop. They used BS to detect moving objects and track them. The authors of [18] have used background subtraction as a case study to demonstrate their approach of performing big video data processing. The authors of [19] propose a video management platform using Hadoop to achieve fast video processing. BS was one of the tasks implemented in the system. In [20], a video splitting algorithm (background subtraction) has been designed and implemented using MapReduce for parallel processing of metadata extraction for surveillance video. In the previously mentioned works, the authors only focused on using the MapReduce model by introducing different implementations of 
CD. The solution presented in this thesis improves the performance by using not only MapReduce but also different sampling techniques to avoid unnecessary computation.

\subsubsection{Data Placement Policy}

Data locality is a crucial factor in improving the performance of MapReduce jobs running on Hadoop clusters. Poor data locality occurs when part of the data is processed locally while the other parts are transferred through the network from remote nodes for processing. This gives rise to the phenomena, called access load imbalance, and congests the network and degrades the overall system performance. The literature discusses two main reasons for access load imbalance: storage load imbalance and heterogeneous clusters. Storage load imbalance occurs because of the dynamic nature of adding and deleting data as well as adding and removing nodes. The cluster heterogeneity leads to access load imbalance when fast datanodes complete processing the local data and try to share the load by transferring remote data from slow datanodes through the network.

Many attempts described in the literature have been trying to improve the performance of distributed file systems through effective data placement policies. The way the data are distributed among the nodes can significantly improve the performance of the running data-intensive applications. This performance improvement can be achieved as a result of load balancing and exploiting data locality to avoid the cost of transferring data over computer networks.

To handle the heterogeneity of a Hadoop cluster, the research described in [80] proposed a data placement scheme that distributes file fragments on the cluster nodes based on their computing capabilities. The proposed mechanism is trying to minimize data movement between slow nodes and fast ones by assigning more fragments to the faster nodes. The mechanism includes two parts. The first part is an algorithm to perform initial distribution for file fragments, while the second is another algorithm to 
redistribute file fragments in case new data are appended or new computing nodes are added. The drawback of this scheme is that it does not satisfy all HDFS requirements, such as replicating input data file fragments to support reliability and availability.

A lightweight extension of Hadoop called CoHadoop was introduced in [78]. CoHadoop extends HDFS to allow collocating related input files at the file system level. The extension maintains the advantages of Hadoop, such as load balancing and fault tolerance. To collocate the related files, a new file property is introduced as well as the block placement policy of Hadoop is modified. The performance study of CoHadoop in the context of log processing shows the improvement achieved by the proposed Hadoop extension [78].

In [81], the authors proposed a distributed algorithm that allows the datanodes of a Hadoop cluster to balance themselves without intervention from the namenode. The goal of the scheme described in this thesis proposal is to solve the load imbalance resulting from the dynamic nature of clouds on which files can be created, appended, and deleted, while nodes can be added, removed, and replaced.

The authors of [79] proposed a dynamic algorithm to balance the load among different racks of a Hadoop cluster based on information collected from Hadoop log files. The algorithm estimates the performance of each rack in terms of processing capacity. The conducted simulation study shows substantial improvement in job execution time.

The algorithm proposed in [82] was devised to deal with different situations that may affect the distribution of the load among the nodes. The situations involve adding and deleting data as well as adding and removing datanodes. To balance the workload as evenly as possible, a new role is designated to a node to help in matching heavy-loaded and light-loaded datanodes. Nodes adopting such a role are termed BalanceNodes.

The replica placement policy proposed in [83] was devised to generate even replica 
assignment and meet HDFS replica placement requirements. The proposed solution, called Partition Replica Placement Policy (PRPP), consists of two phases. The first phase divides all nodes into three sections, and the second phase distributes replicas on those sections. The drawback of PRPP is that it is devised for homogeneous clusters in which all the nodes are identical in terms of computing capabilities. In order to deal with this challenge, the authors introduced an Improved Slot Replica Placement Policy (ISRPP) with some modifications to the two phases [84]. ISRPP was devised to handle both heterogeneous and homogeneous environments.

The authors of [85] proposed a load balancer and a custom block placement policy for heterogeneous Hadoop clusters. The heterogeneity addressed in the paper includes the heterogeneity resulting from that not all disks have the same access speed nor the same available space to store the data. The limitation of this work is that it did not address the heterogeneity in terms of CPU and memory capacities. The authors of [86] proposed a dynamic block placement policy for heterogeneous Hadoop clusters to improve the performance of MapReduce applications. The proposed solution takes into account heterogeneity resulting from differences in both the processing capacity and storage utilization. The simulation results show improvement in the MapReduce performance.

In [87], a data placement policy that relies on the access frequency of data blocks is proposed. The frequency of accessing data is different not only from one file to another but also from one part of a file to another. In other words, some data blocks could be accessed more than the others, which in turn results in access load imbalance between the data blocks of the same file. Therefore, the proposed solution uses a kpartition algorithm to evenly divide data blocks into multiple groups on the basis of access count statistics for each data block. The access counts of the blocks are sorted in non-ascending order list. Then a first-fit heuristic is used to evenly divide the blocks iteratively from the beginning of the list to the end into multiple $\mathrm{k}$ partitions 
that will be distributed to $\mathrm{k}$ storage servers. A custom data placement policy [88] is proposed to minimize data transfer between nodes and nodes in different racks. To minimize data migration, the authors assign the blocks of related video data to specific nodes that will be responsible for processing the data blocks locally.

Although DPP is a popular topic in the literature, the current works do not consider some video data features that can improve the performance of processing the data on Hadoop clusters. The video data placement strategy introduced by [89] is most similar to the goal of this work. The authors have proposed a novel proactive strategy to store the input video data into the cluster nodes in an adaptive manner. The proposed strategy depends on a model that predicts the computing time based on several features, including the video duration, resolution, and frame rate, in addition to the node processing capability. They have proposed an algorithm to initially place the data into the cluster nodes and another one to rebalance the workload during the job execution. Both [89] and this work are utilizing some video features to distribute the video data among the cluster nodes in order to minimize the video data transfer overhead during the job execution (i.e., access load imbalance). However, there are two main differences: The first one is that [89] is tackling the access load imbalance resulted in by processing different video data sizes on a heterogeneous cluster, while this work is tackling the access load imbalance resulted in by the nature of the proposed NU-MR job that may process different video data having the same size on a homogeneous cluster. The second difference is that [89] uses video features such as video duration, resolution, and frame rate, while the proposed work uses a feature called Amount of Change (AoC), which will be described in Section 6.2.2. 


\subsection{Chapter Summary}

In many video processing applications, change detection can be used as an elementary step to detect the region of interest. It also can be used in mobile object tracking, people counting, and crowd monitoring. Since video data are large in size and grow exponentially, they need more storage and computing power for analysis. The aim of this thesis is to introduce different approaches to improve the performance of change detection on Hadoop clusters so that more video data can be processed in a timely manner. Firstly, the performance of running change detection can be improved through the splitting strategy adopted to partition large video files. Secondly, the performance can be improved by using different sampling techniques along with the MapReduce programming model. The advantage of using the sampling techniques is to avoid unnecessary computation in such a way that more video data can be processed in a timely manner. Eventually, the system performance can be improved based on the policy used to distribute the video segments across the cluster nodes. 


\section{Chapter 3}

\section{The Video Splitting Approach}

To process video data in Hadoop clusters, the data need to be uploaded to HDFS. If the size of the video files is larger than HDFS block size, the video files will be segmented into blocks and distributed among the nodes, which in turn damage the container format of the video data $[90,91]$. This chapter introduces two techniques based on sampling to divide large video files into segments and sample the overlapping frames to minimize the overhead. Change detection [2] is used to process the video files segmented using the proposed approach.

Dividing a large video file into segments is adopted by different works in the literature [19,90-92]. According to this Video Splitting strategy (VS), the size of each video segment should be smaller than the HDFS block size. In VS, if the goal is to run a single-frame oriented algorithm on a video file, the video frames will be divided equally among the video segments. This technique is termed as Naive Video Splitting (NVS). However, if the goal is to run a frame-series oriented algorithm such as BS on a video file, the video frames need to be divided equally among the segments with some overlapping frames among the consecutive segments. This technique is named Naive Video Splitting with Overlapping (NVSwO). To the best of our knowledge, little work exists on VS techniques that support the running of frame-series oriented algorithms on Hadoop clusters. The authors of [16] referred to the fact that overlapping some 
frames during the video Splitting is required to run this class of algorithms on Hadoop. In $[17,19]$, the authors used the BS algorithm (frame-series oriented class) in different applications, and they focused on the speedup gained by running the applications on Hadoop, but not VS, and its effect on performance that are investigated in detail in this thesis.

In NVSwO, the overlapping frames are the source of the overhead since the overlapping frames will be processed twice. To reduce the overhead, this thesis proposes two video Splitting techniques based on sampling the overlapping frames for each segment. The main difference between the proposed techniques and the existing NVSwO technique is that the proposed techniques consider a lower number of overlapping frames leading to a significant reduction in processing overhead.

\subsection{The Proposed Video Splitting Strategy}

The BS algorithm needs some history frames that function as a training set to generate the initial background model. When a video file is segmented, each segment needs some overlapping history frames (training set) from the previous segment. The first segment has a training set that does not involve any overlapping frames. NVSwO can be used to segment the video file so that the BS algorithm can be applied. The challenge here is that the more the video is segmented, the more overlapping frames are required. Processing overlapping frames is deemed overhead since those frames will be processed twice. The number of overlapping frames depends on the video content. If the Splitting occurs at a frame where the scene is crowded with more foreground objects or slow-moving objects, more overlapping history frames are required to generate the first background model. This is because the background pixels of the recent history frames are occluded by more foreground objects pixels. Since we do not know whether the Splitting occurs at a crowded frame or not, a 
reasonable number of overlapping history frames (training set) needs to be chosen for a given application. This estimation can be achieved by using the Receiver Operating Characteristics (ROC) curve analysis, as will be described later in Section 3.2.1.

A novel video Splitting strategy called Sampling Video Splitting (SVS) to reduce the overhead of the overlapping frames has been proposed. The idea is that instead of using $h$ consecutive history frames as a training set, $h^{\prime}$ history frames are sampled out of the $h$ history frames. SVS reduces the number of overlapping history frames, which in turn reduces the overhead, especially when the video is divided into a large number of segments to increase the degree of parallelism.

\subsubsection{Video Splitting Modeling}

To detect the foreground objects in a video file $F$ comprising $n$ frames, the first $h$ frames (called the initial history frames) will be used as a training set to estimate the first background model $f_{b g}^{0}$. The first background model is updated by considering the most recent history frames and discarding the old ones. Let $V_{h}$ denote the training set of $F$, while the remaining $m$ frames are called the data frames $\left(V_{d}\right)$. The data frames $V_{d}$ are used to detect the foreground objects as a binary mask image $\left(f_{b m}^{t}\right)$ for each data frame $f_{d}^{t}$ at time $t(0 \leq t<m)$. To detect the foreground objects in a given frame $f_{d}^{t}$, the background model $f_{b g}^{t}$ needs to be estimated from the recent $h$ frames. The background model $f_{b g}^{t}$ is subtracted from the data frame $f_{d}^{t}$ at time $t$ to generate $f_{b m}^{t}$ :

$$
f_{b m}^{t}=f_{d}^{t}-f_{b g}^{t}
$$

According to NVSwO, a video file is divided into $p$ segments $\left\{V^{0}, V^{1}, \ldots, V^{p-1}\right\}$ where $\mathrm{p}$ determines the degree of parallelism. Each segment $j\left(V^{j}\right)$ comprises two types of frames: the training set $\left(V_{h}^{j}\right)$ and the data frames $\left(V_{d}^{j}\right)$ : 


$$
V^{j}=V_{h}^{j} \cup V_{d}^{j}
$$

The training set of a given segment $j$ can be described by:

$$
\begin{gathered}
V_{h}^{j}=\left\{f^{j d}, f^{j d+1}, \ldots, f^{j d+h-1}\right\} \\
\left|V_{h}^{j}\right|=h
\end{gathered}
$$

The frames (from $f^{0}$ to $f^{h-1}$ ) represent the training set for the first segment, where $f^{0}$ is the first frame in the original video. Without losing generality, Figure 3.1 illustrates an example of dividing a video file of 20 frames into two video segments. Figure 3.1 (a) shows that the first 4 frames that are the history training frames while the rest are the data frames. Figure 3.1 (b) shows how the data frames have been partitioned into two segments of 8 frames. The training set for the first segment $V_{h}^{0}$ is similar to the history frame $V_{h}$ of $F$. However, the training set of the second video segment $\left(V^{1}\right)$ is overlapped with the previous segment $V^{0}$ according to Equation 3.3; i.e., $\left\{f^{8}, f^{9}, f^{10}, f^{11}\right\}$. On the other hand, the set of data frames of segment $j$ is given by:

$$
\begin{gathered}
V_{d}^{j}=\left\{f^{j d+h}, f^{j d+h+1}, \ldots, f^{(j+1) d+h-1}\right\} \\
d=\left|V_{d}^{j}\right|=\lceil(n-h) / p\rceil
\end{gathered}
$$

where $\mathrm{d}$ represent the size of video segment $j$.

The data part of the original video file $V_{d}$, which includes $(n-h)$ frames, is divided evenly among the segments. From Equations 3.4 and 3.6, the size of each segment $j$ can be expressed as: 


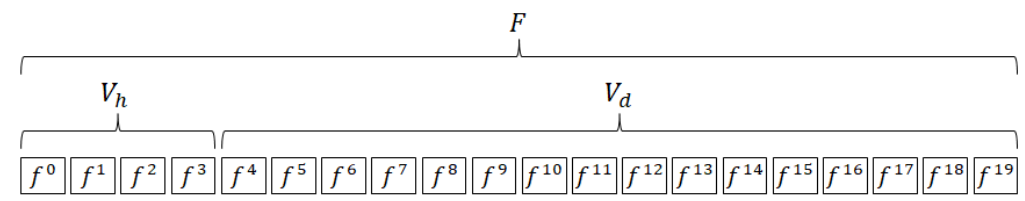

(a)

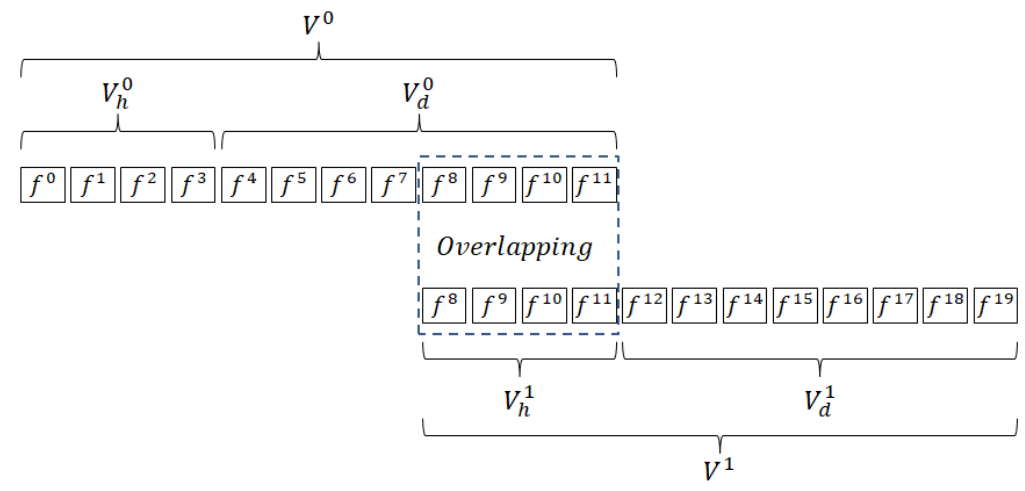

(b)

Figure 3.1: Naive Video Splitting with Overlapping (NVSwO) model.

$$
\left|V^{j}\right|=h+(n-h) / p
$$

The aggregate size for all segments is:

$$
\sum_{j=0}^{p-1}\left|V^{j}\right|=(p-1) h+n
$$

The BS algorithm processes all the $n$ frames of the video sequentially on one node to generate and update the background model. In parallel processing version, there are $(p-1) h+n$ frames to be processed. This means that there are $(p-1) h$ more frames processed. These frames represent the overlapping history frames (training set) for each segment $j>1$. The overhead (in terms of frames) due to processing the overlapping frames twice is denoted by $\alpha$ :

$$
\alpha=(p-1) h
$$


From Equation 3.9, it is clear that the overhead is affected by two factors: the number of segments $p$, and the number of the overlapping history frames $h$. Increasing $p$ or $h$ results in increasing the overhead. Increasing $p$ depends on the required degree of parallelism, while increasing $h$ depends on the required training set size.

\subsubsection{Sampling Video Splitting (SVS)}

This thesis proposes two Sampling Video Splitting techniques (SVS) to reduce overhead $\alpha$. The idea is to sample some frames out of $V_{h}^{j}$ to generate a new training set $S_{h^{\prime}}^{j}$ of size $h^{\prime}$ frames for each segment $j$. The entire segment with sample history $V_{s}^{j}$, the size of each new segment with sample history, the total number of input frames, and the overhead $(\alpha)$ in terms of number of frames resulted in by SVS can be expressed, respectively, as follows:

$$
\begin{gathered}
V_{s}^{j}=V_{h^{\prime}}^{j} \cup S_{d}^{j} \\
\left|V_{s}^{j}\right|=h^{\prime}+(n-h) / p \\
\sum_{j=0}^{p-1}\left|V_{s}^{j}\right|=p h^{\prime}-h+n \\
\alpha^{\prime}=p h^{\prime}-h
\end{gathered}
$$

From Equations 3.9 and 3.13, it is clear that the overhead reduces by $p\left(h-h^{\prime}\right)$ frames (the difference between the two equations). Therefore, the execution time that can be saved because of using the proposed SVS is:

$$
\tau=T\left(p\left(h-h^{\prime}\right)\right)
$$




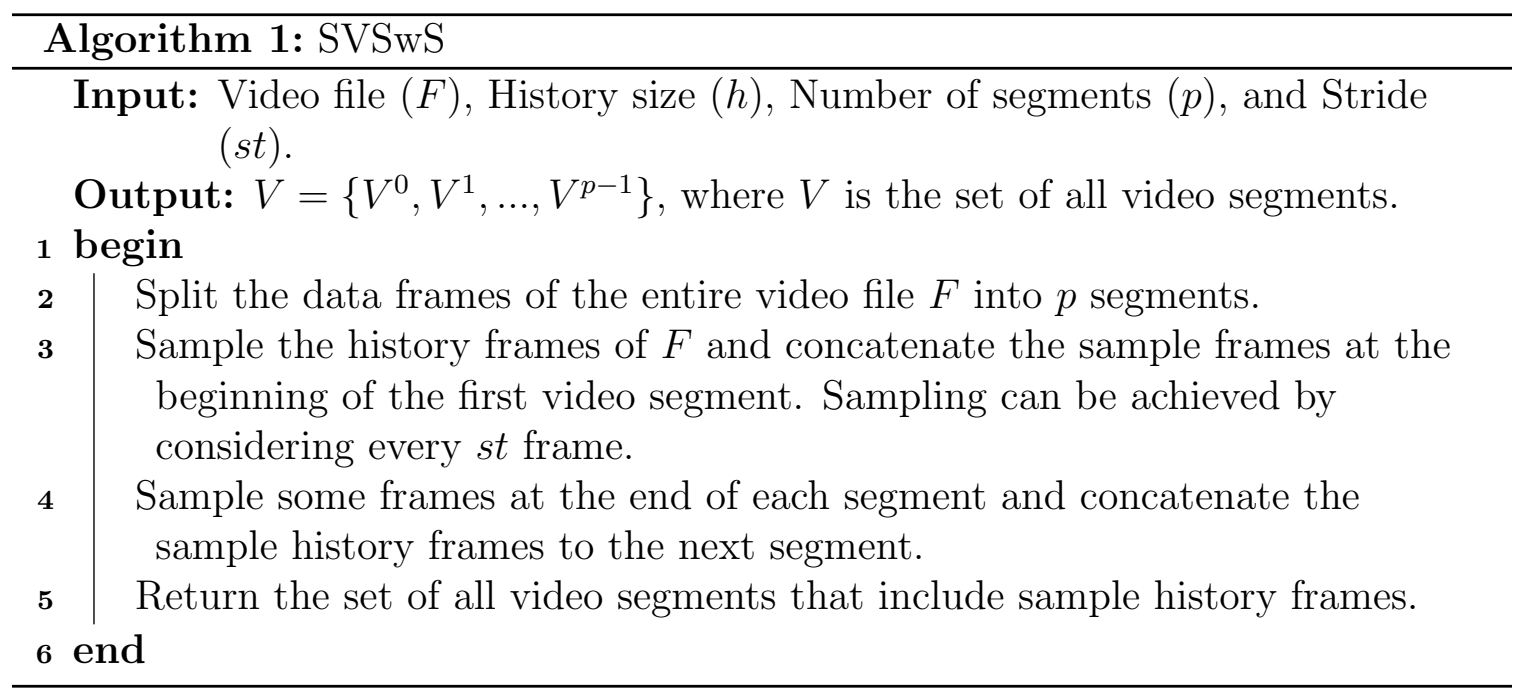

Where $T($.$) is the time function to process a number of frames. The effect of \tau$ on the performance of running the BS algorithm on a Hadoop cluster is shown experimentally in Section 3.2.2.

The first technique is called Sampling Video Splitting with Stride (SVSwS). Algorithm 1 shows the details of SVSwS. According to the algorithm, a large video file is divided into segments, and at the beginning of each segment, some overlapping frames form the previous segment should be sampled except the first segment. The sampling technique is to consider every $i^{\text {th }}$ frame. Further details are included in Appendix A.1.

The second technique is called Sampling Video Splitting with Fibonacci Window (SVSwFW). In SVSwS, the intervals between samples are fixed; however, the goal of using SVSwFW is to use variable intervals between the samples, and this variable interval can be achieved by the Fibonacci pattern. According to SVSwFW, a window of size w slides over the training set $V_{h}^{j}$ to select some frames for the sample training set $V_{h^{\prime}}^{j}$ for the segment. The window is an array that holds the value ' 1 ' at the indices that match the Fibonacci sequence $\{0,1,2,3,5,8, \ldots\}$, otherwise; the array holds '0'. For example, Figure 3.2 shows a window of size 8 that holds the value 


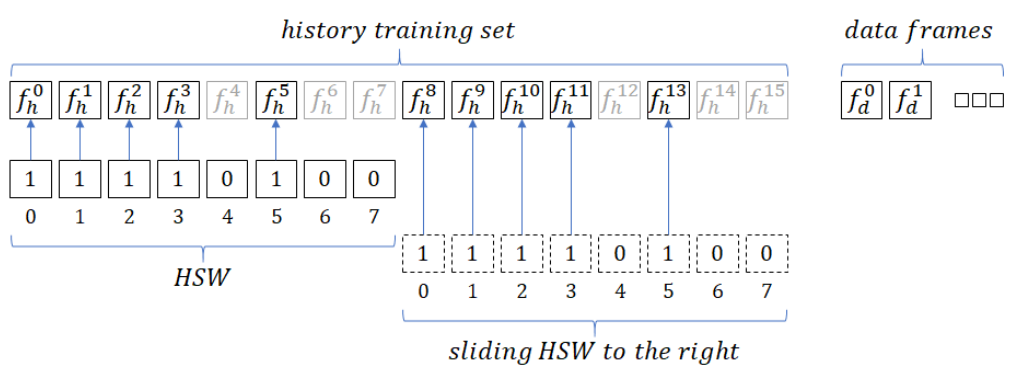

Figure 3.2: Sampling Video Splitting with Fibonacci Window (SVSwFW).

'1' at the indices that match the Fibonacci sequence, $\{0,1,2,3,5\}$, and the value '0' at the other indices, $\{4,6,7\}$. The window starts from the beginning of $V_{h}^{j}$, and all the frames in $V_{h}^{j}$ that correspond to the value ' 1 ' in the window will be added to the sample training set $V_{h^{\prime}}^{j}$. Thereafter, the window slides to the next part of $V_{h}^{j}$ and repeats the same procedure (sampling the frames corresponding to the value '1') until the window slides over the entire $V_{h}^{j}$ (Algorithm 2). Further details are included in Appendix A.2. The characteristic of these techniques is that it considers more frames at the beginning of each window and less frames at the end of the window. This is useful to reduce the error in the estimated initial background model, especially when the Splitting occurs at a crowded scene since more distant history frames will be deemed.

To justify why SVSwFW could show better performance, let us consider the scenario in Figure 3.3. This scenario is taken from Changedetection.net benchmark [21]. As shown in the scenario, a person is moving in the scene, and the goal is to generate a background model from 7 frames out of the first 28 frames. As can be noticed in the first three frames, the background is not covered by foreground moving objects; however, in the following frames, the background is partially covered with the moving person (i.e., the region of interest is partially covered). The importance of using the Fibonacci scheme is that it models the background using 3 frames with no foreground objects (frames 1,2 and 3), and 4 frames that are partially covered 


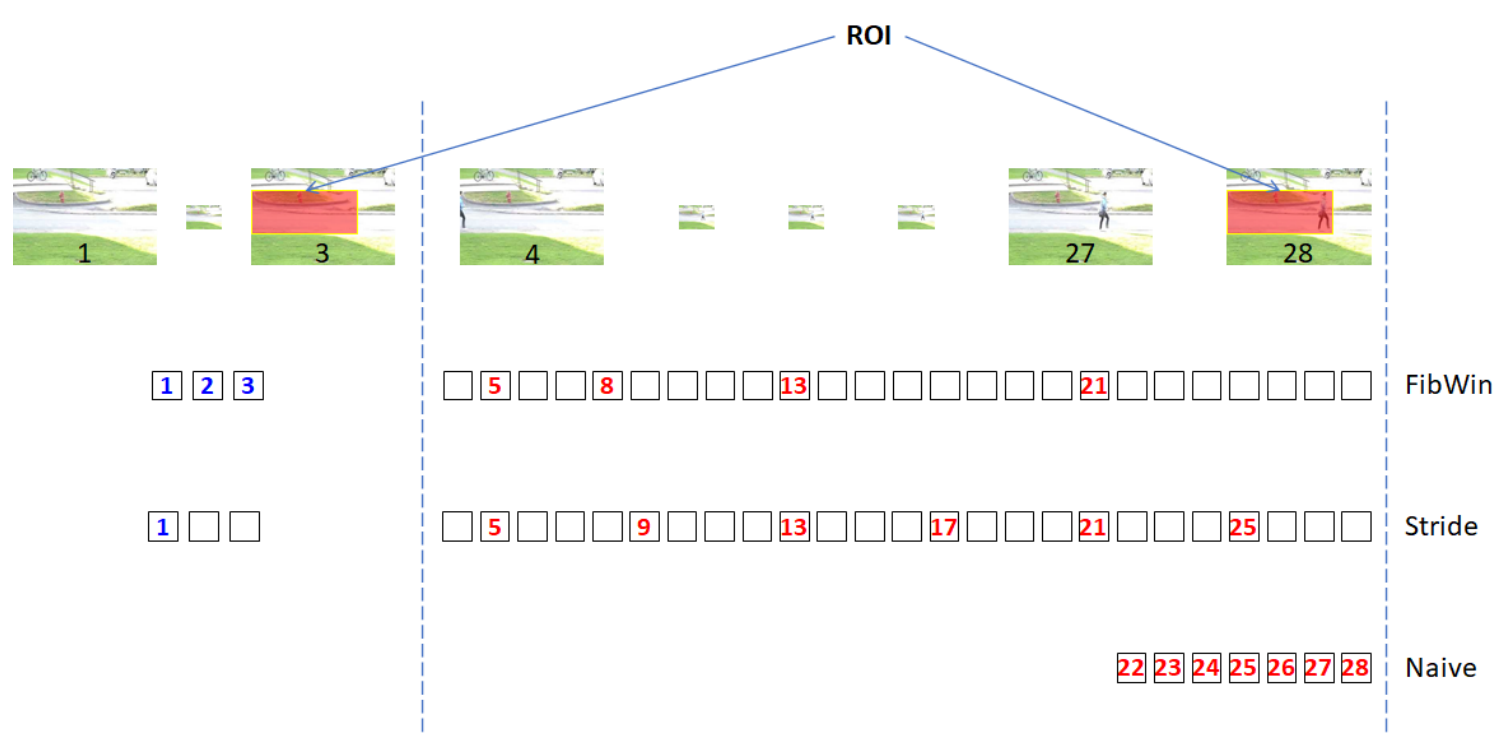

Figure 3.3: Advantage of using Fibonacci Window.

with the foreground object (frames 5, 8, 13 and 21). When the stride technique is used, there is one frame (frame 1) without foreground objects, and 6 frames ( frames $5,9,13,17,21$ and 25) covered partially with the moving object. When the naive technique is used, all the seven frames are partially covered with the moving object, hence giving rise to lower accuracy in comparison to that achieved with using Fibonacci-based sampling. Increasing the number of history frames to generate the initial background model significantly improves the accuracy of the naive technique, especially with more slow-moving objects covering the scene. However, processing more overlapping frames (overhead) degrades the performance. In the case of using the Fibonacci-based technique, the advantage is that reducing the number of history frames to generate the initial background model minimizes the overhead and does not significantly degrade the accuracy, even if there are moving objects covering the background. 


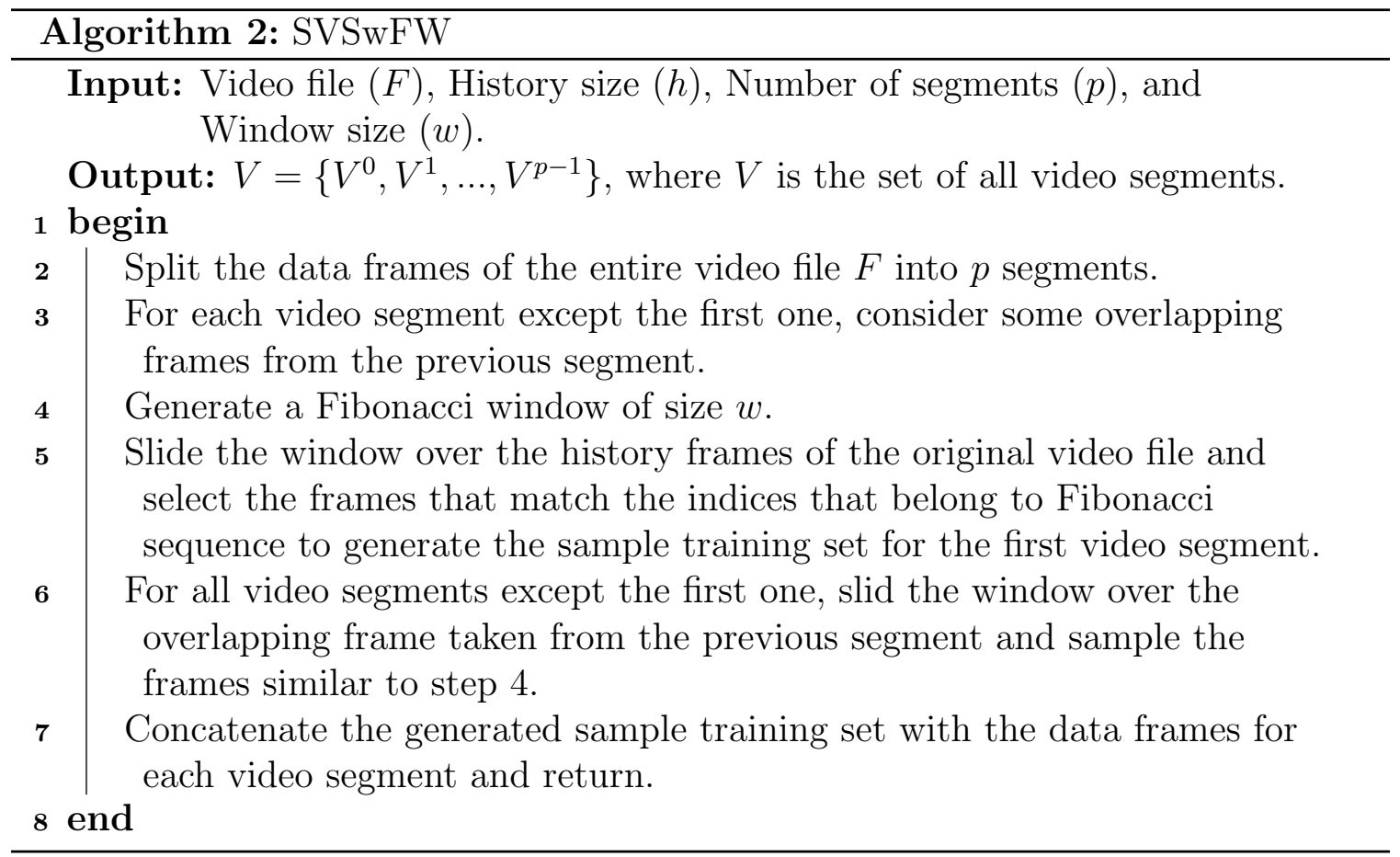

\subsubsection{MapReduce-based Change Detection}

To run the CD algorithm [2] on video files stored in HDFS, the MapReduce programming model is used. A MapReduce program uses InputFormat and RecordReader to access the data stored in HDFS. Since Hadoop does not support reading video data, InputFormat and RecordReader have been extended using Java classes called VideoFileInputFormat and VideoFileRecordReader, respectively. VideoFileRecordReader transforms the video data into key-value pairs $<$ FrameNo, FrameData $>$ to be processed by the map tasks. FrameNo is LongWritable that represents the offset of the frame from the beginning of the video. To represent FrameData, we implemented the Writable interface using a java class called FrameWritable. The output of the CD algorithm is the foreground binary masks for all the input frames. The output can be written back to HDFS from the map tasks since only map tasks are used in this case. 


\subsection{Experiments}

This section discusses the accuracy and the performance of running the MapReducebased BS algorithm on a video file segmented using the proposed techniques.

\subsubsection{Accuracy Evaluation}

The accuracy metrics described in [2] to evaluate the quality of the BS algorithm are used. The idea is to compute the binary label image and then compare it with a ground truth mask at the pixel level. A ground truth mask is a binary image provided with the dataset designed to test the accuracy of BS algorithms. The ground truth is obtained by direct observation and used to check the validity of the output of computer vision algorithms. From the comparison, the following statistics can be computed: (1) number of true positives (TP) or the number of correctly classified foreground pixels, (2) number of true negatives (TN) or the number of correctly classified background pixels, (3) number of false positives (FP) or the number of incorrectly classified foreground pixels, and (4) number of false negatives (FN) or the number of incorrectly classified background pixels. The two metrics used in this thesis are:

$$
\begin{aligned}
& \text { TruePositiveRate }(T P R)=\frac{T P}{T P+F N} \\
& \text { FalsePositiveRate }(F P R)=\frac{F P}{F P+T N}
\end{aligned}
$$

The Receiver Operating Characteristic (ROC) curve is used to plot the (TPR/FPR) pairs for different thresholds that decide whether a pixel belongs to the background or the foreground objects. Better performance is obtained when the curve approaches the top edge $(\mathrm{TPR}=1)$ and the left edge $(\mathrm{FPR}=0)$. When $\mathrm{TPR}=1$, 
it means that the probability of detection is 1 (i.e., all foreground pixels are detected correctly). When FPR $=0$, it means that the probability of false detection is 0 .

In this study, three datasets are used: two datasets from a benchmark called Changedetection.net [21], and a recorded dataset prepared for this evaluation. From the changedetection.net benchmark, PETS2006 and the highway datasets are used, Figures 3.4 and 3.6, respectively. The PETS2006 dataset includes a recording of people moving in a public area for a period of time. The dataset is modified by scaling up the size of the frames from $720 \times 576$ pixels to 1920x1536 pixels and repeating the sequence many times to increase the number of frames from 1200 to 18900 . The modified dataset will be called Modified PETS2006. The reason for modifying the dataset is to increase its size so that it can benefit from the use of Hadoop, which is designed to deal with big data. The second dataset is a video recording of skaters for 10 minutes 30 seconds recorded using a video camera (Figure 3.5). This recording represents an outdoor scene where people are moving (skating, walking, and running). CD can be used in different applications in this scenario, such as counting the people, tracking them, or monitoring their behavior. The video comprises 18900 frames with 1080P resolution. The first 30 seconds (900 frames) are used as a training set for the CD algorithm for both the benchmark dataset and the skating video. An annotation tool called GNU Image Manipulation Program (GIMP) [93] is used to generate the ground truth for some frames from the recorded video. 


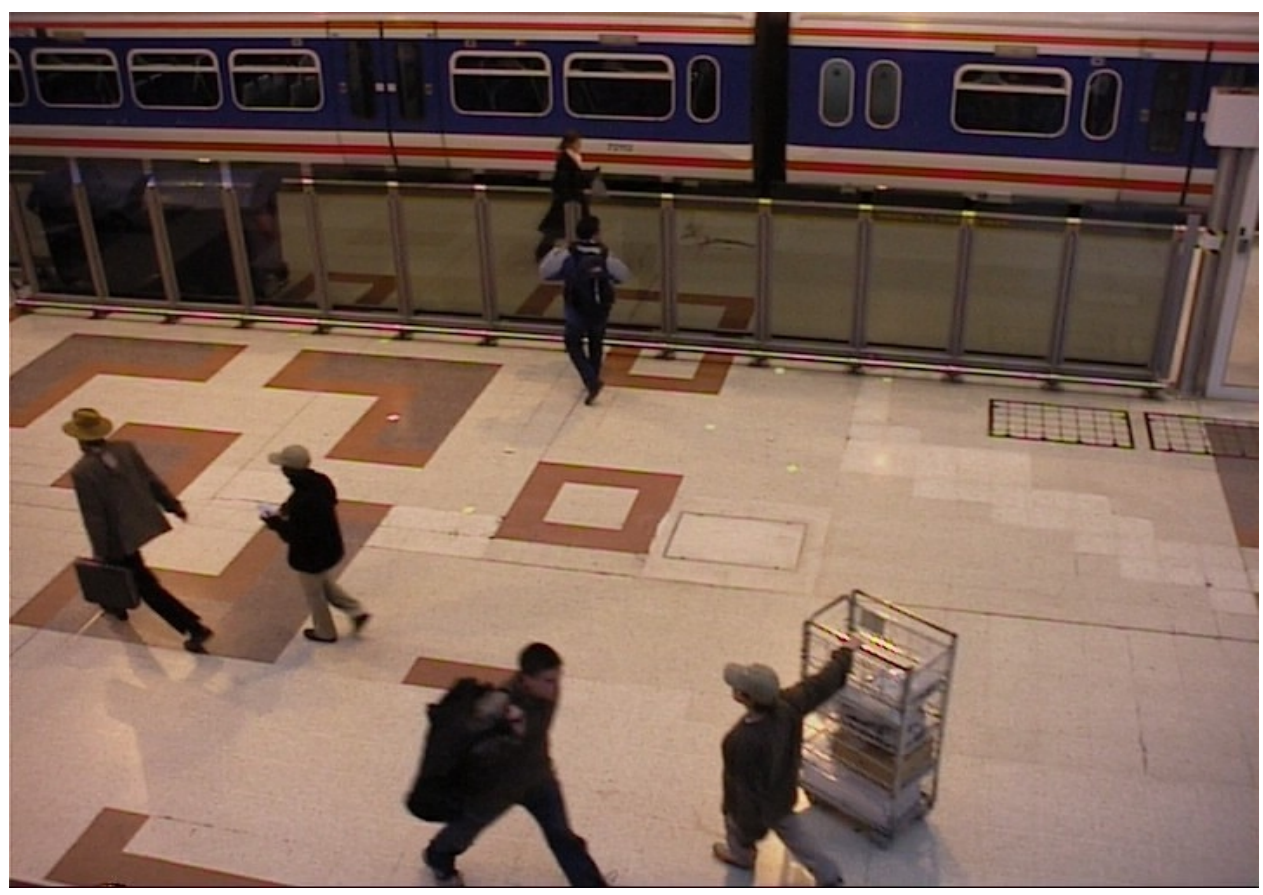

Figure 3.4: PETS2006 dataset.

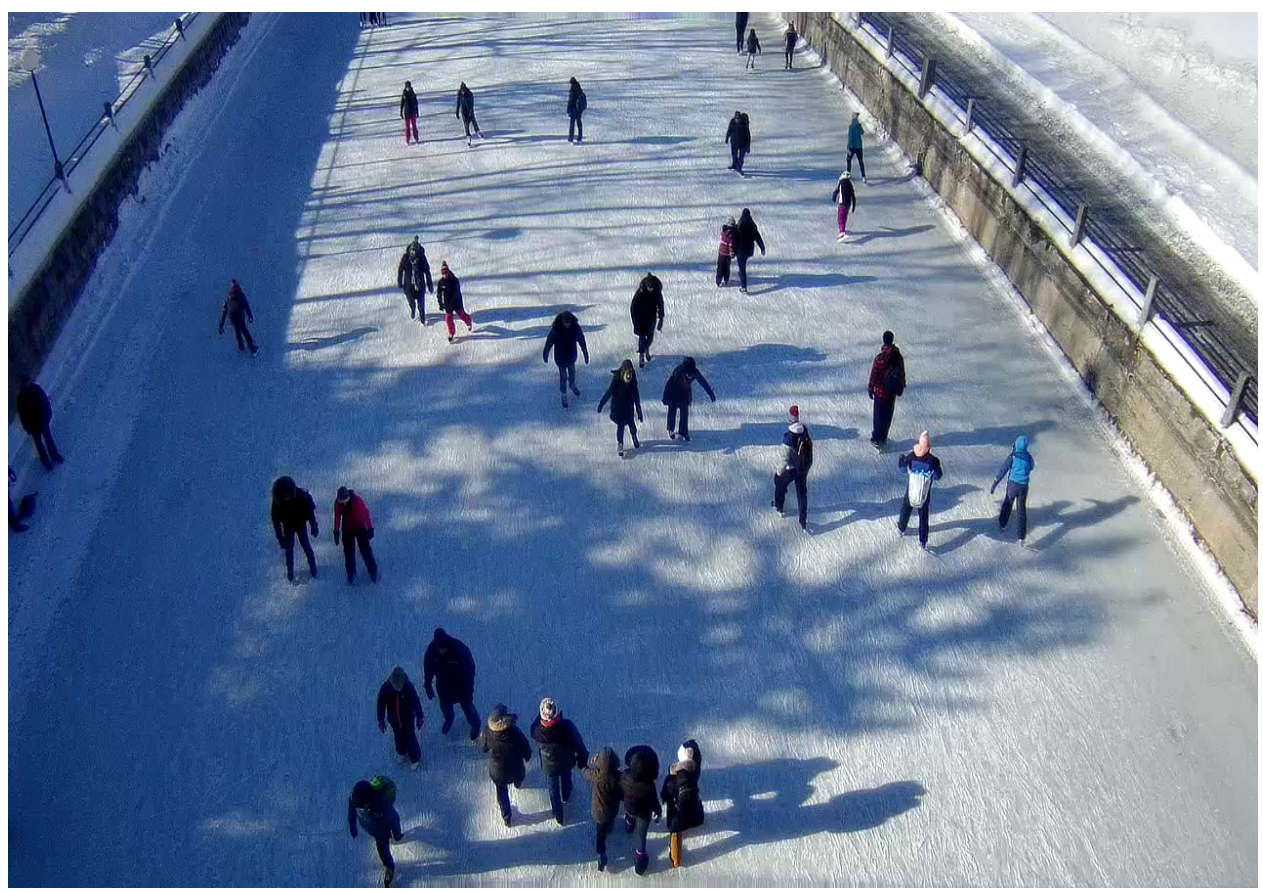

Figure 3.5: Skating dataset. 


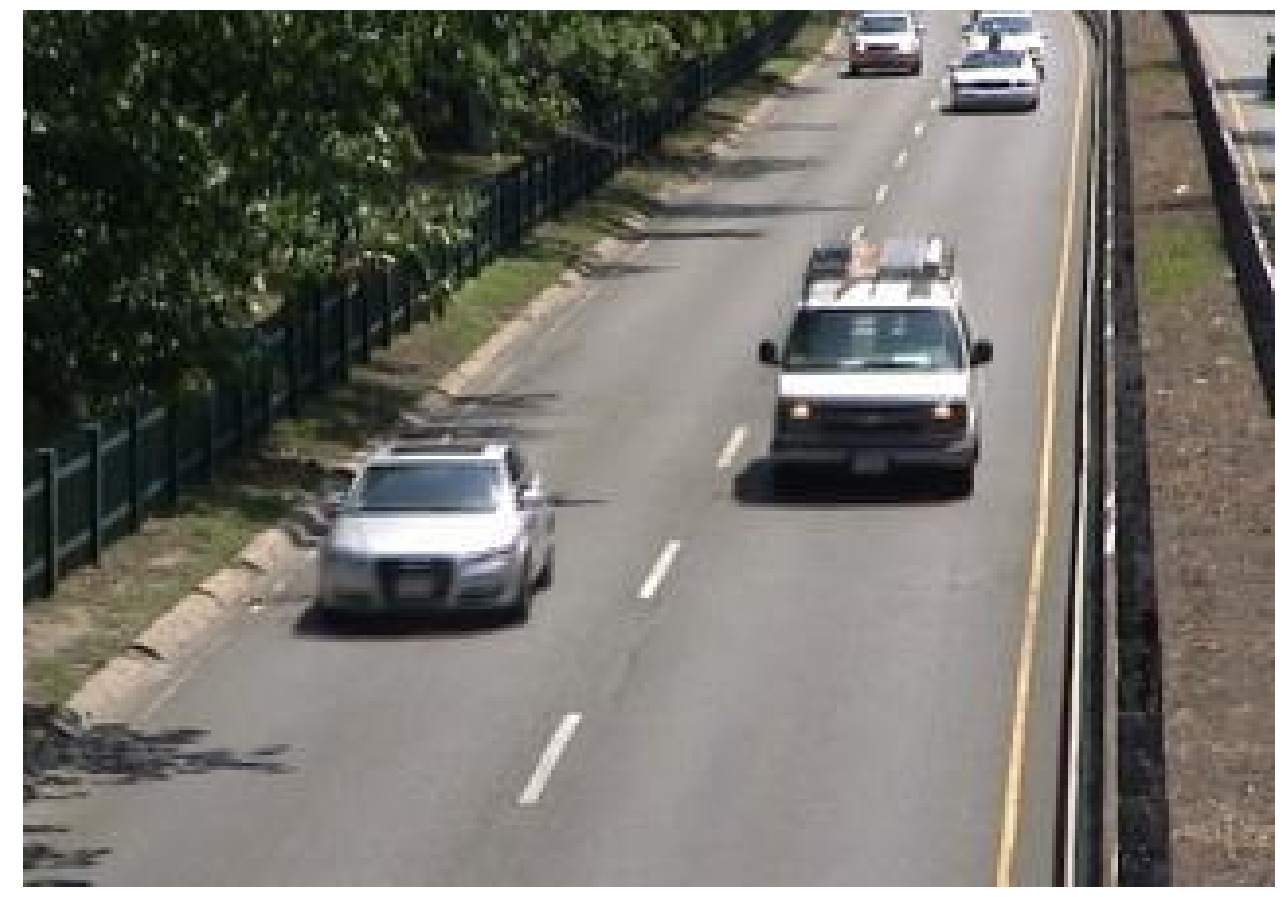

Figure 3.6: Highway dataset.

Dividing a video file into segments requires a training set $V_{h}^{j}$ so that the BS algorithm can process each segment independently on a different node. Since the first data frame for each segment $f_{d}^{0}$ is the most affected frame by the video Splitting, the accuracy test will focus on detecting the foreground objects in the first data frame. In all accuracy tests, the plotted results are the accuracy of computing the foreground binary mask for the first data frame $f_{b m}^{0}$.

The parameters used in the quality evaluation for all techniques are shown in Table 3.1. In NVSwO, the number of history frames used in the training set is varied according to the table. For SVSwS, the stride parameter is fixed at 3, and the number of the sample history frames used in the training set is varied. For SVSwFW, the window size parameter is varied according to the table, and the history size parameter is fixed at 900 frames. The third parameter, the size of the sample frames used as a training set, is determined automatically by the window size and the history size 
parameters. For example, if the window size is 55, and the history size is 900 , the number of sample frames used in the training set is 151 frames, as discussed in Section (3.1.2).

Table 3.1: Accuracy Evaluation Parameters.

\begin{tabular}{ccc}
\hline Parameter & Value & Default \\
\hline \hline Sampling history size $\left(h^{\prime}\right)$ & $10,27,100,300,900$ & 27 \\
Stride & $3,5,7$ & 3 \\
Window size $(w)$ & $55,89,610$ & 610 \\
\hline
\end{tabular}

For each experiment, there are multiple ROC curves. Each curve represents the accuracy of using a different training set size. Each point on a curve represents a different threshold value by which the BS algorithm determines whether a pixel is a foreground or background pixel. The history size varies from a small number to a large number to show that increasing the history size improves the accuracy. The history size 27 is used to allow comparing NVSwO with NVSwFW that uses a window size of 610 frames that allow sampling 27 frames. The window size parameter is used with NVSwFW, and the selected values are three consecutive numbers from the Fibonacci sequence to show the effect of the window size on the accuracy.

\subsubsection{Modified PETS2006 Dataset}

The ROC curve for the traditional video Splitting technique (NVSwO) shows that the larger the training set, the higher is the accuracy. The accuracy results for NVSwO are shown in Figure (3.7). The x-axis represents FPR, while the y-axis represents TPR. The figure demonstrates that as the history size increases, the performance of NVSwO improves. Increasing the number of frames to estimate the background model 
gives rise to the following trade-off: the better the accuracy, the longer the execution time. The statistics collected from experiments are shown in detail in Appendix B, Table B.1.

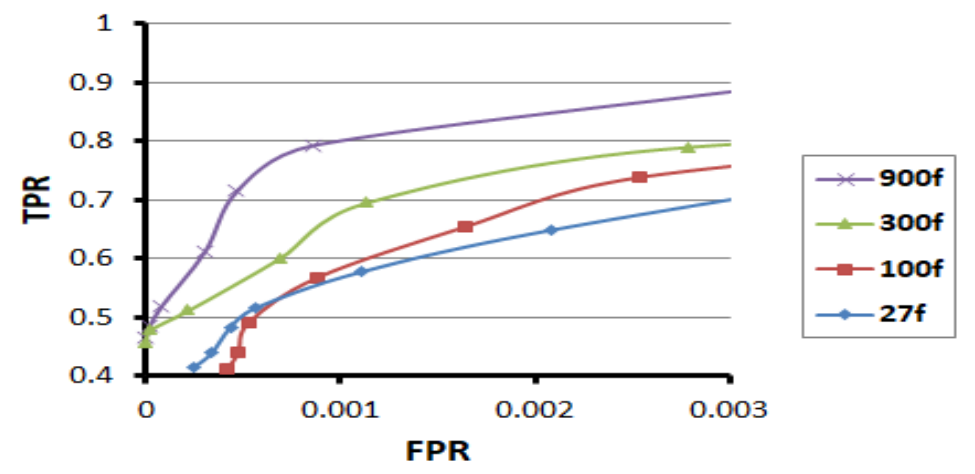

Figure 3.7: NVSwO (PETS2006).

The ROC curve (Figure 3.8) shows the effect of using SVSwS, where every third frame is considered in sampling. This technique needs a smaller training set to achieve accuracy close to that of NVSwO. For example, with a 300-frame training set, SVSwS gives an accuracy close to that achieved with NVSwO and a 900-frame training set. This is because of the ability to consider frames from longer history in case the very recent history frames have background pixels occluded by foreground objects. Table B.2 in Appendix B shows the statistics of this experiment.

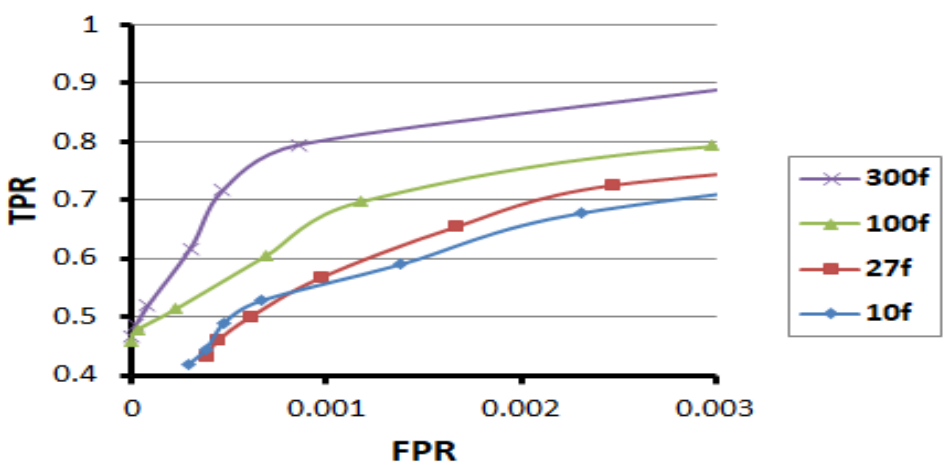

Figure 3.8: SVSwS (PETS2006). 
The ROC curve in Figure (3.9) illustrates the effect of the stride parameter on the performance of SVSwS. The history training set includes 27 sample frames, where every $3^{\text {ed }}, 5^{\text {th }}$, and $7^{\text {th }}$ are considered. The results show that sampling with every $3^{\text {ed }}$ frame is better than the other values in this scenario. Table B.3 in Appendix B gives the details collected from running these experiments. Increasing the stride value improves the accuracy when there are moving objects in the background, and will be discussed in the skating and highway scenarios in Sections 3.2.1.2 and 3.2.1.3, respectively. The value of $s$ that gives the best accuracy may depend on the scenario. In this scenario, there is a person moving slowly or standing within a limited area in the scene, so increasing the stride value will not improve accuracy. In this experiment, using the stride value 'three' gives slightly better accuracy since more sampled frames have been taken while the object is moving.

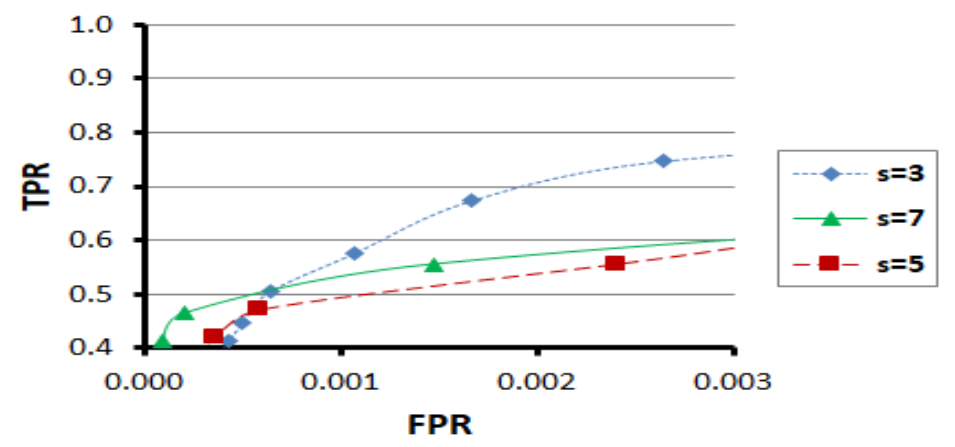

Figure 3.9: SVSwS with different strides (Modified PETS2006).

The ROC curve (Figure 3.10) shows that SVSwFW needs a small training set to generate the background model. Furthermore, using different window sizes leads to almost the same accuracy. For instance, using a window of size 610 (that samples 27 frames) shows very close results to that achieved with a window of size 89 (that samples 106 frames), and a window of size 55 (that samples 151 frames). Table B.4 in Appendix B reveals more details about the experiments. 


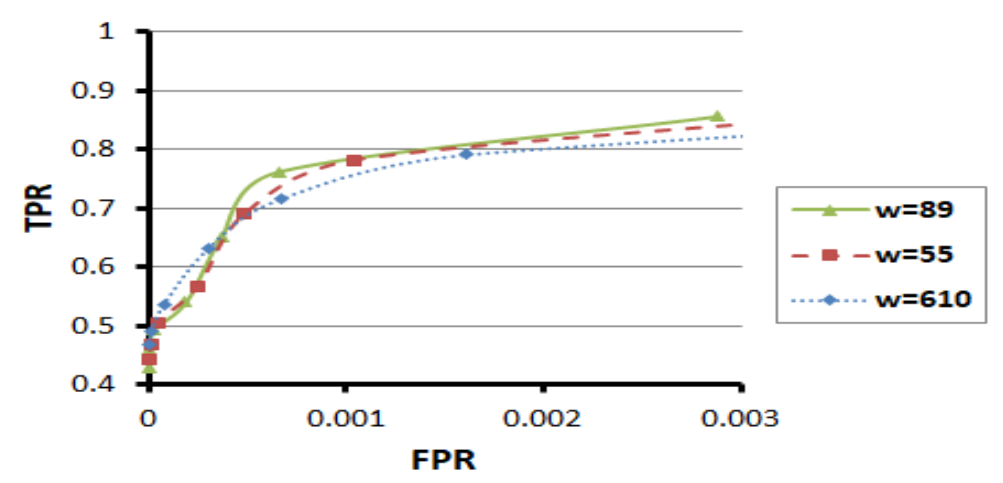

Figure 3.10: SVSwFW (Modified PETS2006).

Figure 3.11 shows that SVSwFW outperforms both NVSwO and SVSwS. This is because if the Splitting occurs at a frame crowded with slow-moving foreground objects, the recent history is more likely crowded with the same foreground objects, especially when a recording with a high frame rate is considered. SVSwFW allows us to sample fewer frames as a training set from a long history, which in turn reduces the effect of slow-moving objects. For example, if there are many moving objects in recent history, this technique will consider more frames from relatively distant history.

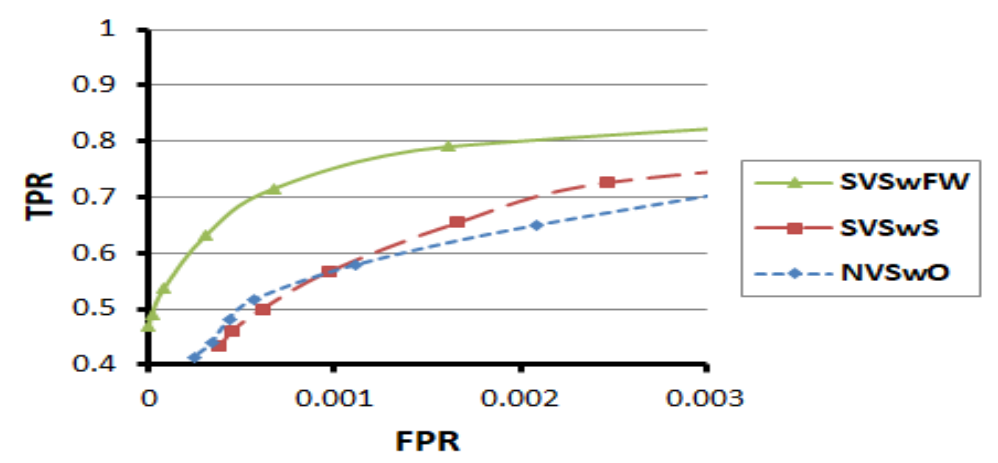

Figure 3.11: Comparing NVSwO, SVSwS and SVSwFW (Modified PETS2006). 


\subsubsection{Skating Dataset}

The accuracy observations for the skating video are similar to those observed with the modified PETS2006 dataset. The ROC curve in Figure (3.12) depicts FPR (x-axis) against TPR (y-axis). As the training set increases, the accuracy of NVSwO improves. For instance, the accuracy when 900 frames are used to model the background is significantly higher than using 27 frames. Table B.5 in Appendix B reveals more details about the experimental results.

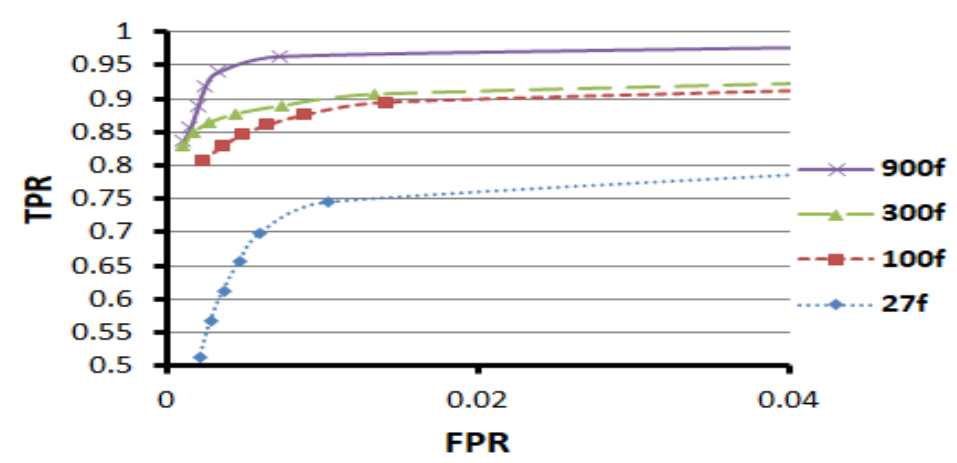

Figure 3.12: NVSwO (Skating).

Figure (3.13) shows the effect of increasing the training set size on SVSwS that samples every $3^{\text {ed }}$ frame. As the results illustrate, the larger the training set, the better accuracy is attained. For example, the accuracy of using 300 sample frames is significantly better than the accuracy of using 10 sample frames. More experimental results are depicted in Appendix B, Table B.6. 


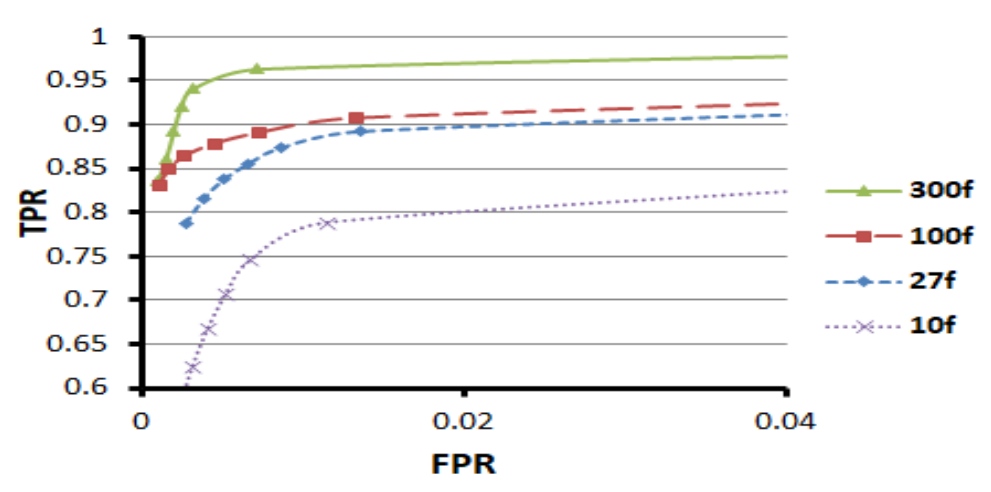

Figure 3.13: SVSwS (skating).

Figure (3.14) depicts the effect of different stride parameter values on SVSwS by sampling every $3^{\text {ed }}, 5^{\text {th }}$, and $7^{\text {th }}$ frames. In this experiment, the sample training set includes 27 sample frames (the default value). The results exhibit no significant difference as the stride variable has been varied. More experimental results are depicted in Appendix B, Table B.7.

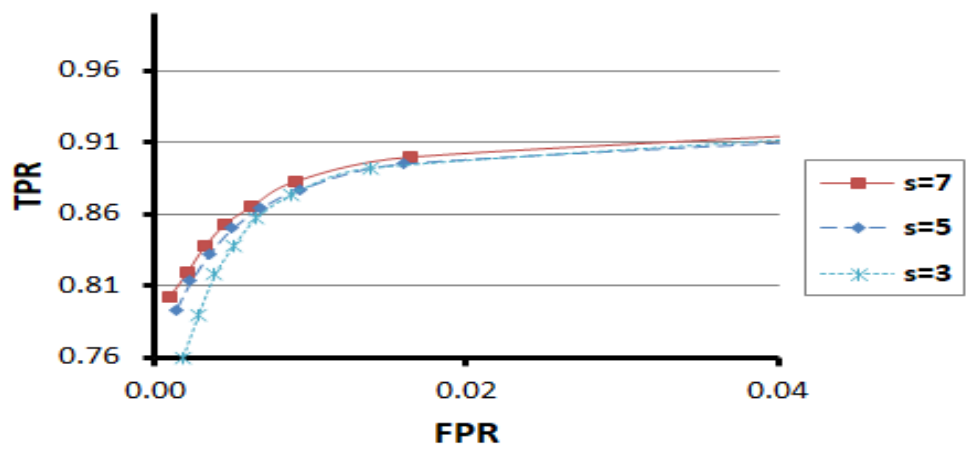

Figure 3.14: Using SVSwS with different strides (Skating).

The accuracy of SVSwFW, as the window size increases, is depicted in Figure (3.15). The results exhibit good accuracy performance regardless of the selected window size. Table B.8 in Appendix B demonstrates the details of this experiment. 


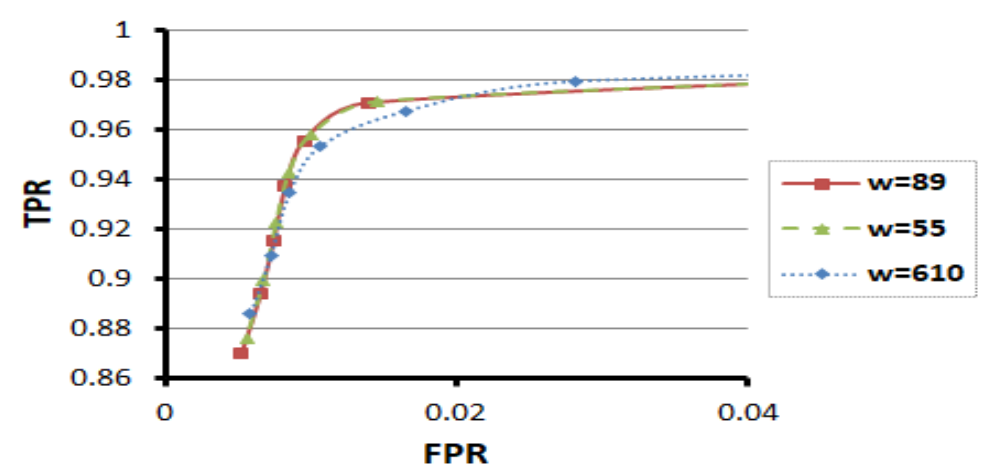

Figure 3.15: Using SVSwFW (skating scenario).

The comparison of the three techniques (NVSwO, SVSwS, and SVSwFW) when 27 frames are used in the training set is revealed in Figure (3.16). The results exhibit that the accuracy of SVSwFW is significantly better than SVSwS, and the accuracy of SVSwS is significantly better than NVSwO.

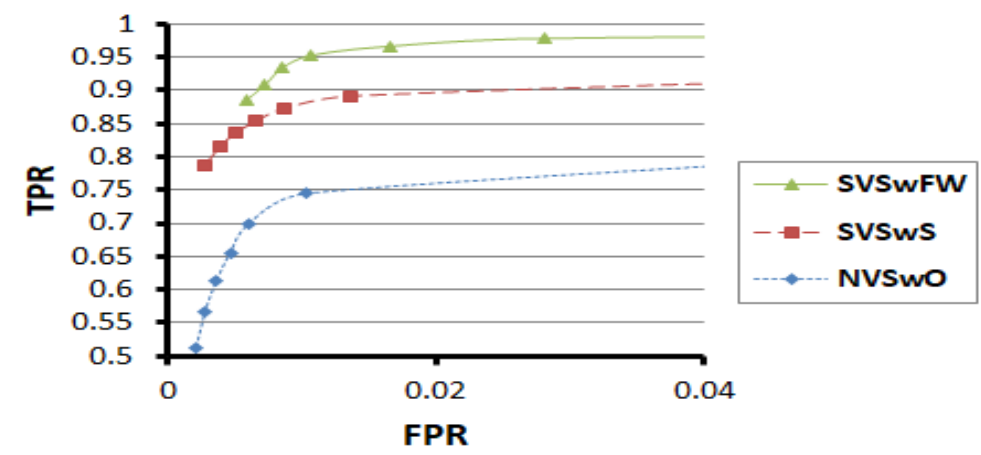

Figure 3.16: Comparing NVSwO, SVSwS and SVSFW (skating).

\subsubsection{Highway Dataset}

The highway dataset shows performance similar to the previous datasets. The ROC curve in Figure (3.17) represents FPR on the x-axis and TPR on the y-axis. Increasing the training set size leads to improving the accuracy of NVSwO. The performance improves at a high rate when the training set size is small, but when the training set 
is becoming larger, no significant improvement has been observed. For example, the accuracy improves significantly when 100 frames are used as a training set with respect to using 27 frames. However, there is no significant accuracy improvement when 900 frames are used in comparison to using 300 frames. More details are illustrated in Appendix B, Table B.9.

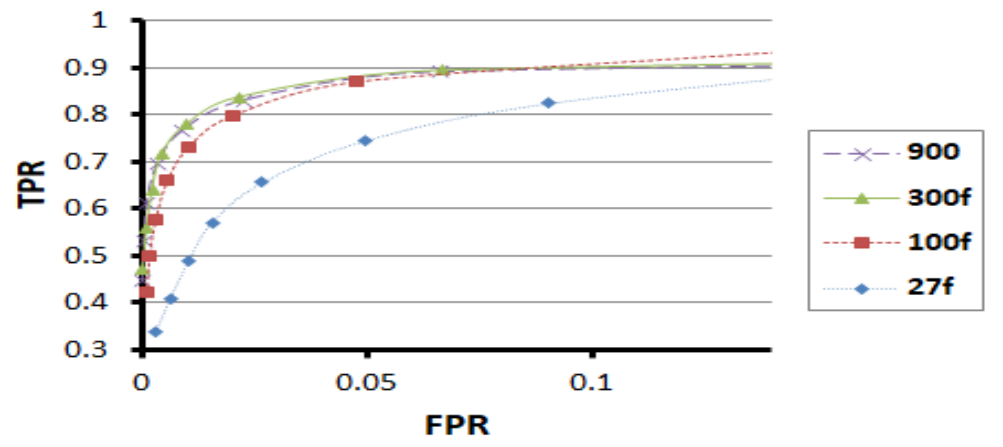

Figure 3.17: NVSwO (Highway).

Figure (3.18) reveals the effect of increasing the training set size on SVSwS with a sampling of every $3^{e d}$ frame. With a small training set (e.g., 10 frames), the accuracy improves faster as the number of sample frames increases. However, as the training set size increases, no significant improvement has been observed. For example, the performance of using a 100-frame training set is similar to the performance of using a 300-frame training set. More details are captured in Appendix B, Table B.6. 


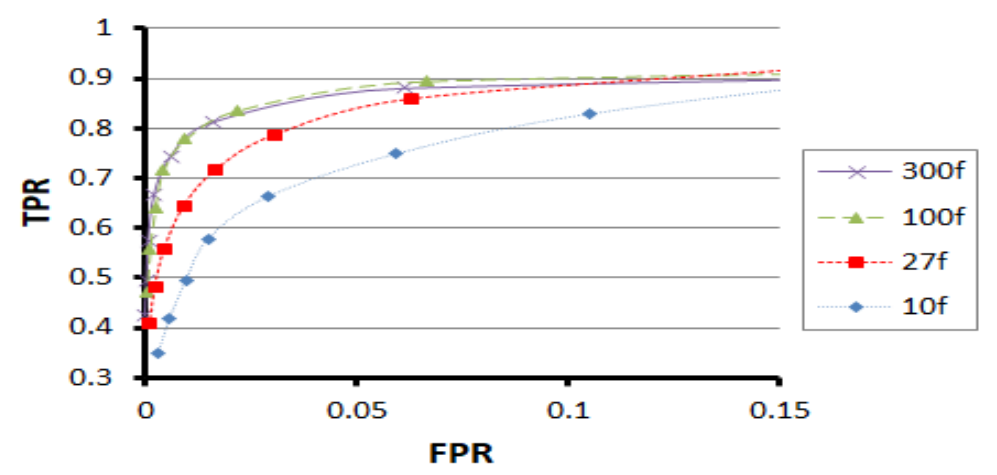

Figure 3.18: SVSwS (Highway).

Figure (3.19) shows the effect of varying the stride parameter on SVSwS by considering every $3^{e d}, 5^{\text {th }}$, and $7^{\text {th }}$ frames. The results reveal that using a larger stride value results in no significant improvement. The detailed results are shown in Appendix B in Table B.11.

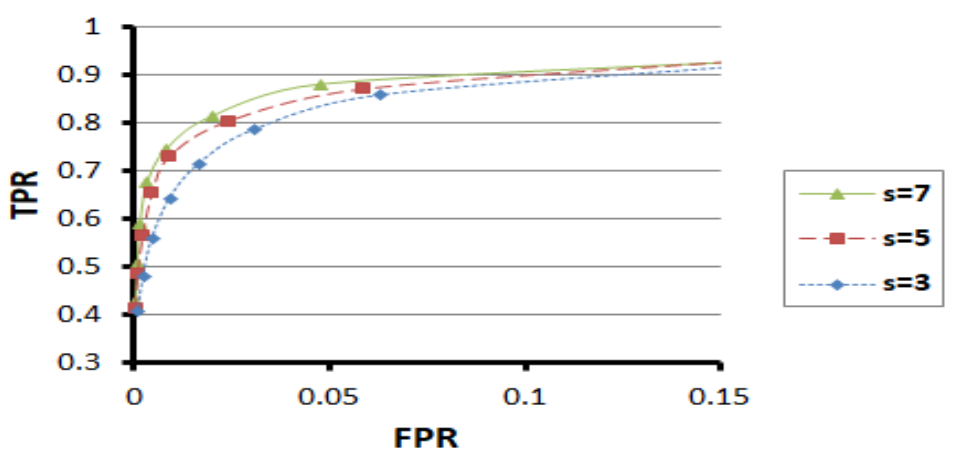

Figure 3.19: Using SVSwS with different strides (Highway).

The accuracy of SVSwFW using different window sizes is depicted in Figure (3.20). The results exhibit that using smaller window size results in a larger sample training set and better accuracy. For example, using a window of size 55 frames or 89 frames gives a better performance than the case in which a window of size 610 frames is used. Table B.12 in Appendix B shows the details of this experiment. 


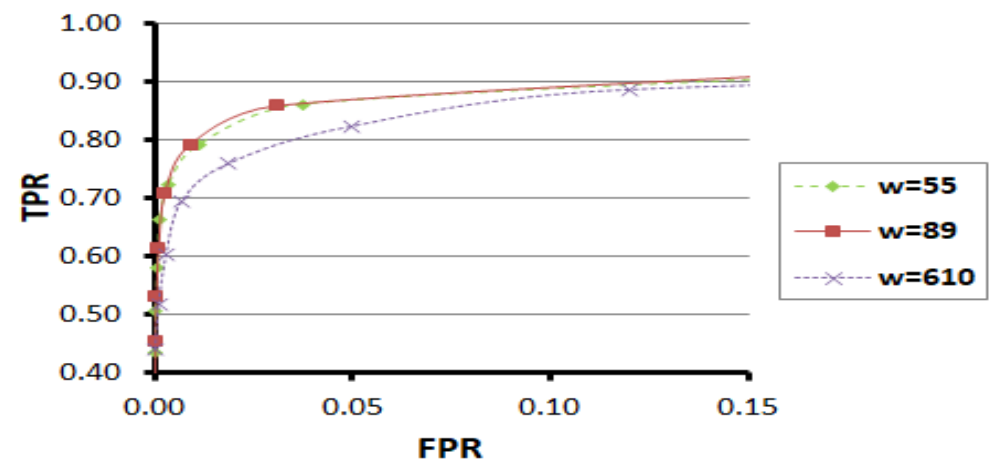

Figure 3.20: SVSwFW (Highway).

Comparing the three techniques is illustrated in Figure (3.21). The results show that the accuracy of SVSwFW and SVSwS is significantly better than NVSwO.

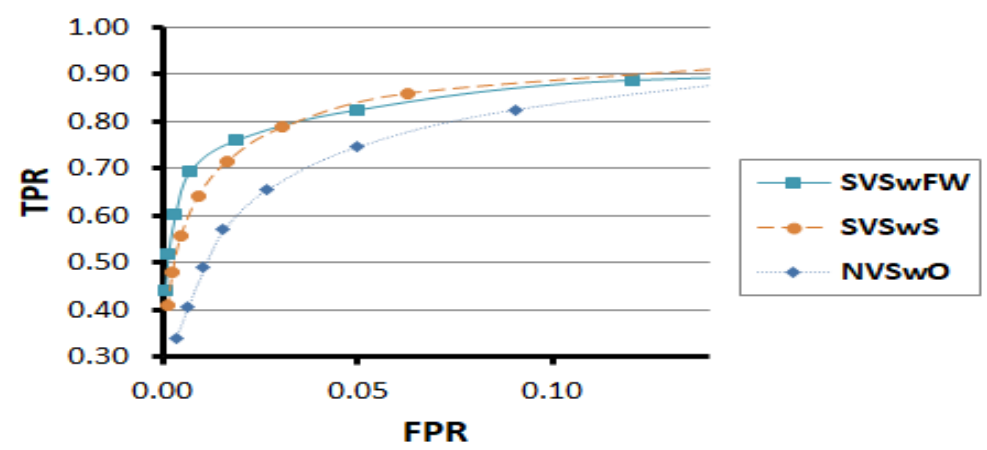

Figure 3.21: Comparing NVSwO, SVSwS and SVSFW (Highway).

\subsubsection{System Performance}

This section analyzes the performance of running the MapReduce-based BS algorithm on a video file segmented using SVSwFW, while NVSwO is used for a comparison purpose. SVSwS demonstrates a performance similar to SVSwFW, so its experimental results are not depicted. Three performance metrics are used to compare the parallel performance of SVSwFW with NVSwO: the execution time, parallel speedup, and 
efficiency. The execution time is the time taken to complete the MapReduce job. The parallel speedup and efficiency are defined as follow:

$$
\begin{gathered}
S_{p}=\frac{T_{1}}{T_{p}} \\
E_{p}=\frac{T_{1}}{p \times T_{p}}
\end{gathered}
$$

where $p$ is the number of nodes, $S_{p}$ is the speedup when $p$ nodes are used, $T_{1}$ is the execution time of running the algorithm on one node, $T_{p}$ is the execution time of running the same algorithm in parallel on $p$ nodes, and $E_{p}$ is the efficiency of running the algorithm on $p$ nodes.

\subsubsection{Datasets}

Both the datasets (the modified PETS2006 and the skating datasets) have been used in the performance evaluation, and they show similar behavior. The modified PETS2006 sequence was decoded into a video file using the FFMPEG tool [22]. The video sequence of modified PETS2006 consists of 18900 frames of 1920x1536 pixels resolution, while the resolution of the skating dataset is 1920x1080 pixels. The first 900 frames represent the training set $\left(V_{h}\right)$ for the video data, while the remaining 18000 frames represent the data frames $\left(V_{d}\right)$.

\subsubsection{Used Technology and Cluster Setup}

To divide a video file into segments, the FFmpeg library is used. OpenCV, the open-source library for computer vision applications, is used to implement the video processing application. Since OpenCV is designed for $\mathrm{C} / \mathrm{C}++$ native applications, JNI is used to wrap the native library so that java can interface to OpenCV native code for writing MapReduce programs that can process video files. 
A 7-node Hadoop cluster was setup up on Amazon EC2. One node was configured to function as a master that runs the name node, secondary name node, resource manager Hadoop daemons. The rest of the nodes were configured as slaves, where each node runs two Hadoop daemons: a node manager and a data node. The t2:large amazon EC2 instances (2 virtual Intel Xeon CPU up to $3.0 \mathrm{GHz}$ clock speed and 8 GB Memory) for all nodes were used. Each video segment is processed by a different map task running on a different node. If a video file is divided into p segments, $\mathrm{p}$ map tasks running on $\mathrm{p}$ different nodes will be participating in processing the video segments. Each experiment is repeated three times, at least, and the average execution time is used in the performance analysis. The measurements were within $\pm 5 \%$ of the average. For example, when the experiments were running on one node (serial execution), they were repeated three times to achieve the desired accuracy. As the degree of parallelism increases, a larger number of repetitions (five times, for example) were often performed for achieving the accuracy goal.

\subsubsection{Experimental Results and Discussion}

To evaluate the speedup achieved by running the BS algorithm on a Hadoop cluster, a set of experiments including processing the video file on 1, 2, 4, and 6 nodes are conducted. Processing a video file on more than one node requires segmenting the file. In these experiments, the video file is segmented using the proposed SVSwFW and the traditional NVSwO for comparison. The training set for each video segment created by using NVSwO is 900 history frames. On the other hand, the training set for each video segment created by using SVSwFW is 27 frames sampled out of 900 history frames. Figure 3.22 shows that, as expected, by increasing the level of parallelism, the execution time decreases for both video Splitting techniques; however, the proposed Splitting SVSwFW outperforms NVSwO as the number of nodes increases. 


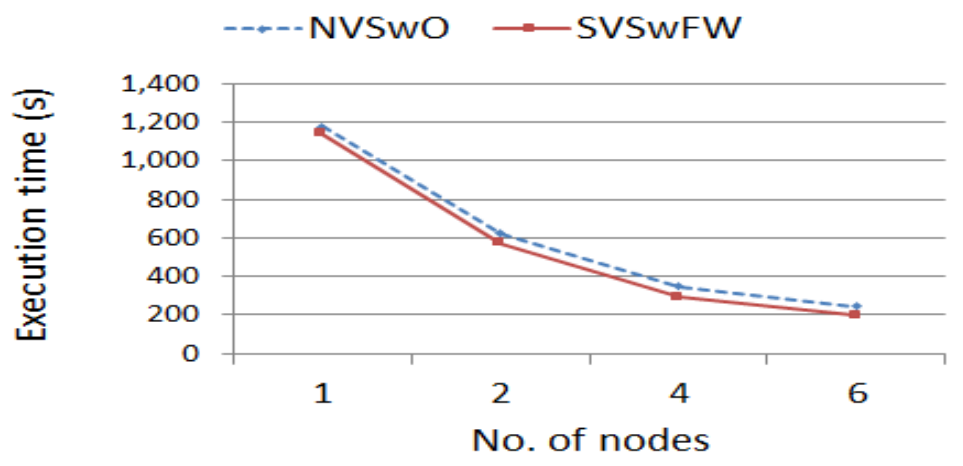

Figure 3.22: The effect of SVSwFW and NVSwO on the execution time (PETS2006 dataset).

Figure 3.23 demonstrates that when SVSwFW is used to segment the video file, the speedup achieved with the MapReduce-based BS algorithm is higher than that achieved with NVSwO.

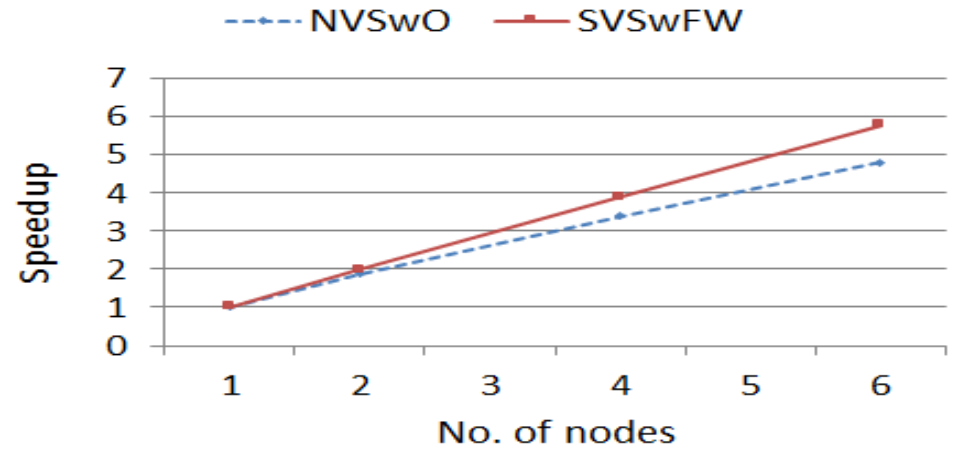

Figure 3.23: The effect of SVSwFW and NVSwO on the speedup (PETS2006 dataset).

As shown in Figure 3.24, the efficiency of the MapReduce-based BS algorithm using SVSwFW is higher than that achieved by running the same algorithm on the video file using NVSwO. As expected, when NVSwO is used, as the degree of parallelism increases, the system efficiency degrades faster than that achieved with the proposed technique (SVSwFW). 


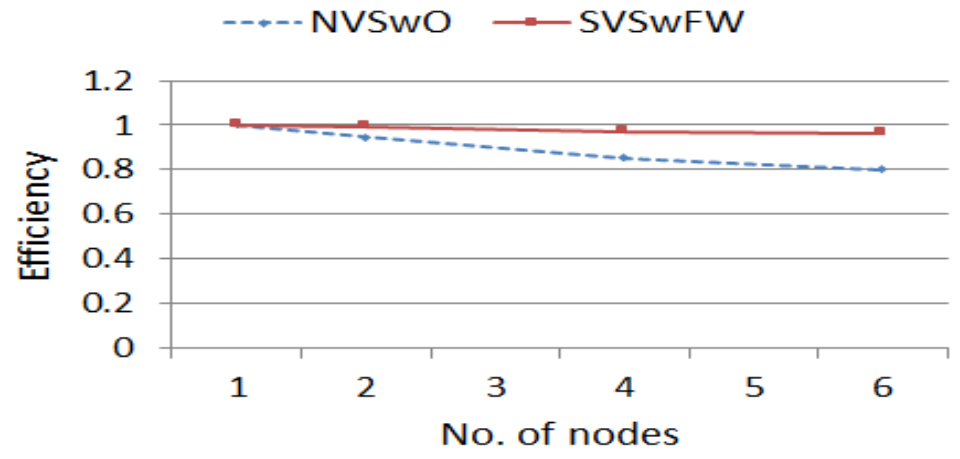

Figure 3.24: The effect of SVSwFW and NVSwO on the efficiency (PETS2006 dataset).

The skating dataset shows similar behavior in terms of execution time, speedup, and efficiency, as shown in Figures 3.25, 3.26, and 3.27, respectively.

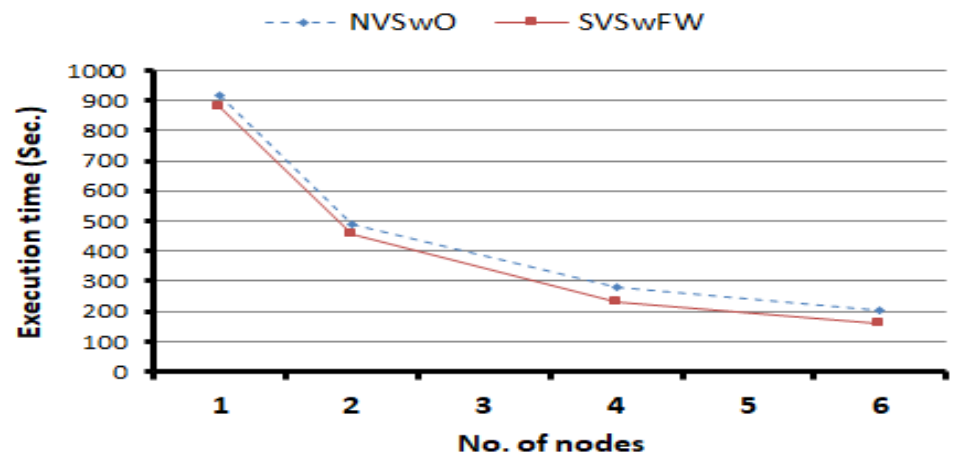

Figure 3.25: The effect of SVSwFW and NVSwO on the execution time (skating dataset). 


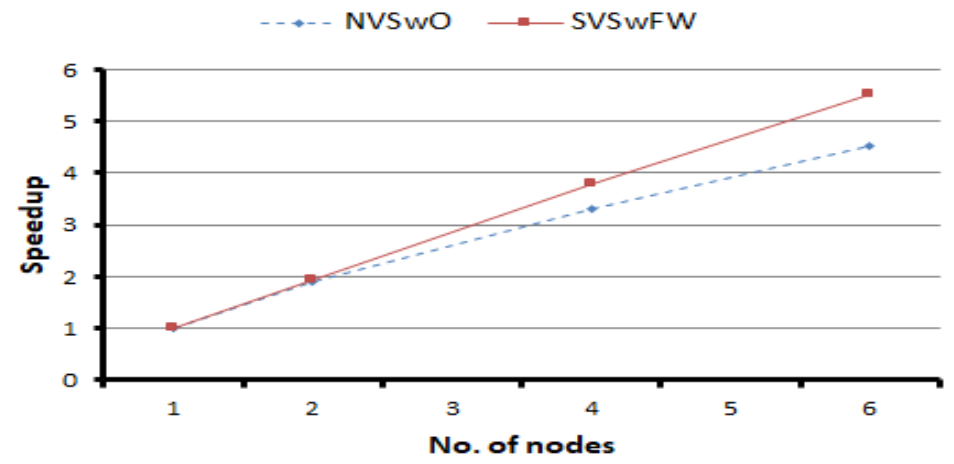

Figure 3.26: The effect of SVSwFW and NVSwO on the speedup (skating dataset).

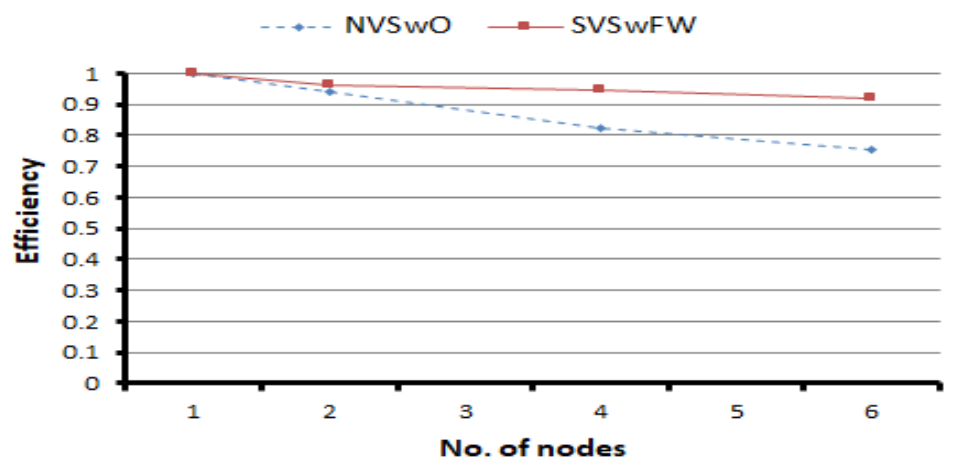

Figure 3.27: The effect of SVSwFW and NVSwO on the efficiency (skating dataset).

\subsection{Chapter Summary}

This chapter introduces a video Splitting strategy that supports both frame-series oriented and single-frame oriented video processing. In frame-series oriented algorithms such as BS, dividing a large video file requires overlapping some frames between every two consecutive segments. Those overlapping frames represent overhead since they are processed twice. The proposed strategy depends on sampling the overlapping frames to reduce the overhead. The technological innovation introduced in this research includes two techniques to implement the strategy: SVSwS, and SVSwFW. 
Furthermore, proof of concept prototypes for these techniques were built and run on a Hadoop cluster to demonstrate the viability and efficiency of the approach. The quality evaluation showed that SVSwFW outperforms SVSwS and the traditional video splitting technique (NVSwO). The performance evaluation in terms of execution time, speedup, and efficiency showed that the proposed video Splitting technique (SVSwFW) outperforms the traditional technique (NVSwO). SVSwFW exhibits a higher speedup than NVSwO. The experimental results demonstrate that the performance of running video processing applications on Hadoop clusters is affected not only by the used algorithms and the input size of video data but also by the way that the video files are segmented and stored on HDFS. The proposed approach for segmenting a video file with a sampled training set (SVSwFW) improves the system speedup without significant quality loss compared to segmenting the same video file with a longer training set used by the traditional technique (NVSwO). 


\section{Chapter 4}

\section{A Change Detection Approach Based on MapReduce and Sampling}

Video analytics is becoming more useful in many video surveillance applications; however, the data requires large amounts of space to be stored, and significant computing power for processing. Fortunately, while processing video data, it is not necessary to process all the video frames, but we can process some of the frames from which the required results can be extracted. This chapter introduces an approach to improve the performance of change detection by using the MapReduce programming model to process the video data in parallel and to use a sampling method to avoid processing unnecessary video frames. The chapter includes an accuracy evaluation to demonstrate the effect of the proposed solution on the result obtained after applying the technique and performance investigation to demonstrate the reduction in execution time.

\subsection{Sampling-based Change Detection Approach}

The goal of change detection is to generate a binary mask that segments a video frame into white pixels that signify the foreground objects and black pixels that signify the 
background. The approach introduced in this chapter uses GMM proposed by [2]. GMM uses $h$ frames to train the initial background model. After that, the model is subtracted from the current frame to detect the foreground objects as a binary mask. Because the algorithm is adaptive, it updates the background model by considering the recent history frames and discarding the old ones.

The proposed approach to speed up the change detection algorithm (SdCD) comprises of two phases. The first phase is based on using MapReduce to parallelize the processing. To do so, large video data should be equally distributed among the nodes of a Hadoop cluster to achieve the best possible performance. The second phase depends on sampling that uses double thresholds to reduce the amount of processing in each Hadoop node. The idea is that instead of updating the background model with every frame in a video sequence, a double-threshold-based technique has been designed to decide whether to consider every frame in the video sequence or only some of the frames (sample frames). The technique updates the background model with every frame in the video sequence only if the amount of change in the scene exceeds a predefined high threshold. On the contrary, the background model is updated with every $i^{\text {th }}$ frame in the video sequence when the AoC drops under a predefined low threshold. Computing AoC will be described in Section 4.1.3.

\subsubsection{The MapReduce-Based Method}

The first phase of SdCD is to use MapReduce to distribute the computation over multiple nodes of a Hadoop cluster. In order to process video data on the cluster, the video files initially have to be uploaded to HDFS. One important consideration is that the size of each video file has to be smaller than the size of HDFS data block (or data block for short). This means that any video file larger than the data block size requires to be divided into segments so that each one is smaller than the data block size. To process the uploaded video data, a MapReduce-based program needs 
to be devised to read the data, process them, and write the output back to HDFS in parallel.

The responsibility of reading the video data in parallel is assigned to the map tasks running on different datanodes. The map tasks read the data from HDFS as keyvalue pairs $<$ FrameNo, FrameData $>$. FrameNo denotes the offset of a particular frame from the beginning of the video sequence, and its data type is LongWritable. FrameData represents the content of one video frame. The data type of FrameData is FrameWritable, which is a new data type created by implementing the Hadoop Writable interface. To read the video frames as key-value pairs, InputFormat and RecordReader are used. Since there are no InputFormat nor RecordReader to handle the video data, their functionalities need to be extended [13]. InputFormat and RecordReader in this work have been extended using java classes named VideoInputFormat, and VideoRecordReader, respectively. Covering the implementation details of extending the Hadoop classes and interfaces is not important in the context of this thesis that focuses on research. The output of SdCD is a set of binary masks for all the input frames. The proposed implementation is based on map-only tasks to improve the performance, so no reduce task(s) are required. Eventually, the goal of the first phase of SdCD is to reduce the execution time by utilizing the parallelism of the MapReduce programming model.

\subsubsection{The Traditional Change Detection Method}

To process big video data $V$ on a Hadoop cluster, the data should be partitioned into segments that are to be processed in parallel. The traditional approach to process the video data (without sampling) using the MapReduce programming model will be referred to as Simple Change Detection (SimpleCD) [16-20]. This approach is considering every frame in the training set to estimate the initial background model. As shown in Algorithm 3, the algorithm receives a video segment and the size of 
the training set as input to estimate the initial background model as output. It iterates over all the training set frames to update the initial background model (lines 3-6) before returning the initial background model (Line 7). Thereafter, SimpleCD uses all the data frames to update the background model and compute the foreground binary mask frames, as shown in Algorithm 4. The algorithm inputs comprise a video segment to be processed, the training set size, and the estimated initial background model. The resulting output is the set of all binary mask frames that refer to the detected changes.

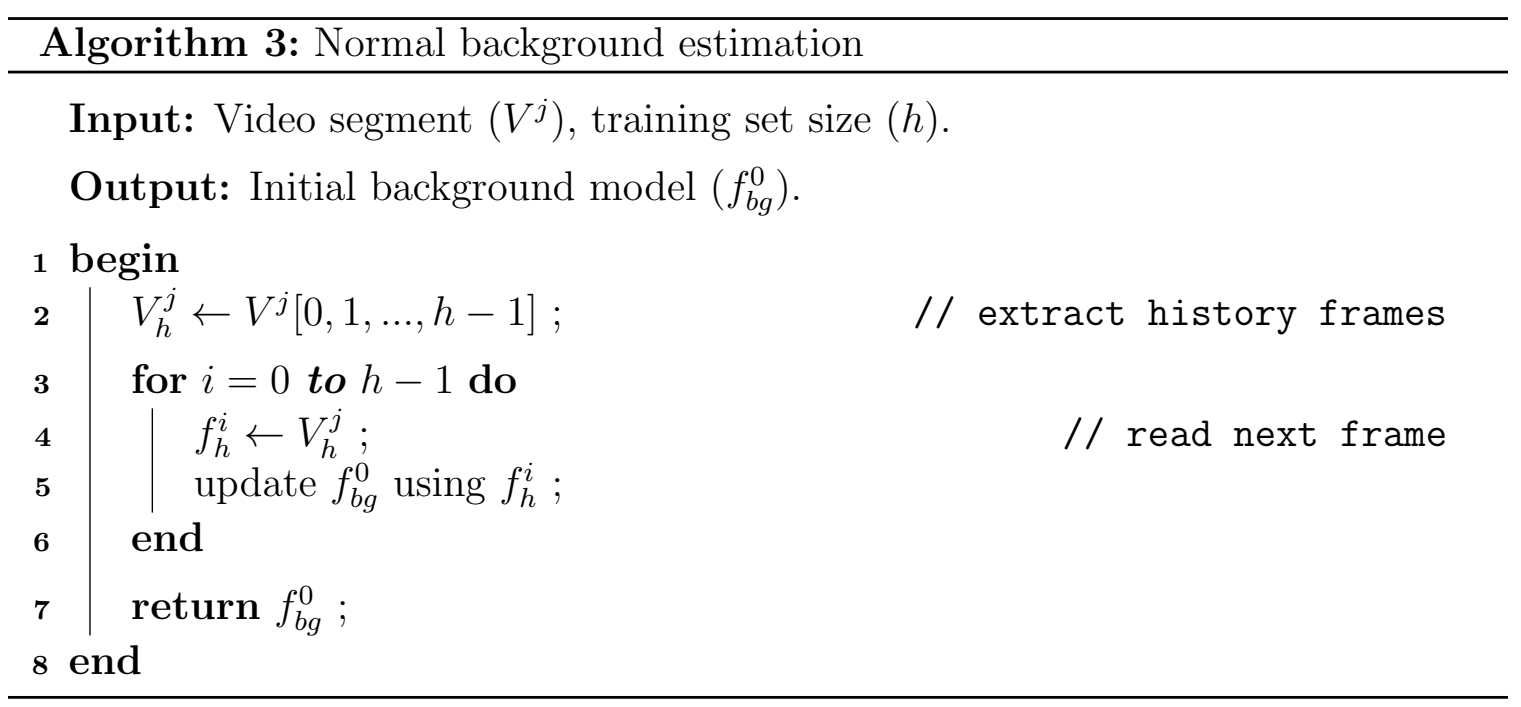

\subsubsection{The Proposed Sampling Method}

The second phase of the proposed SdCD technique is to use sampling to reduce the computation used for updating the background model with each frame in the video sequence. The sampling algorithm initially requires $h$ frames (a training set) starting from the beginning of a video sequence $j\left(V^{j}\right)$ to generate the initial background model. The remaining $d$ frames are the data frames that will be subtracted from the updated background model to generate the foreground binary mask frames (or foreground frames for short). A foreground frame refers to the background pixels 
Algorithm 4: Normal background model update

Input: Video segment $\left(V^{j}\right)$, training set size $(h)$, initial background model $\left(f_{b g}^{0}\right)$.

Output: The set of all binary mask frames detecting the changes $\left(V_{b m}^{j}\right)$.

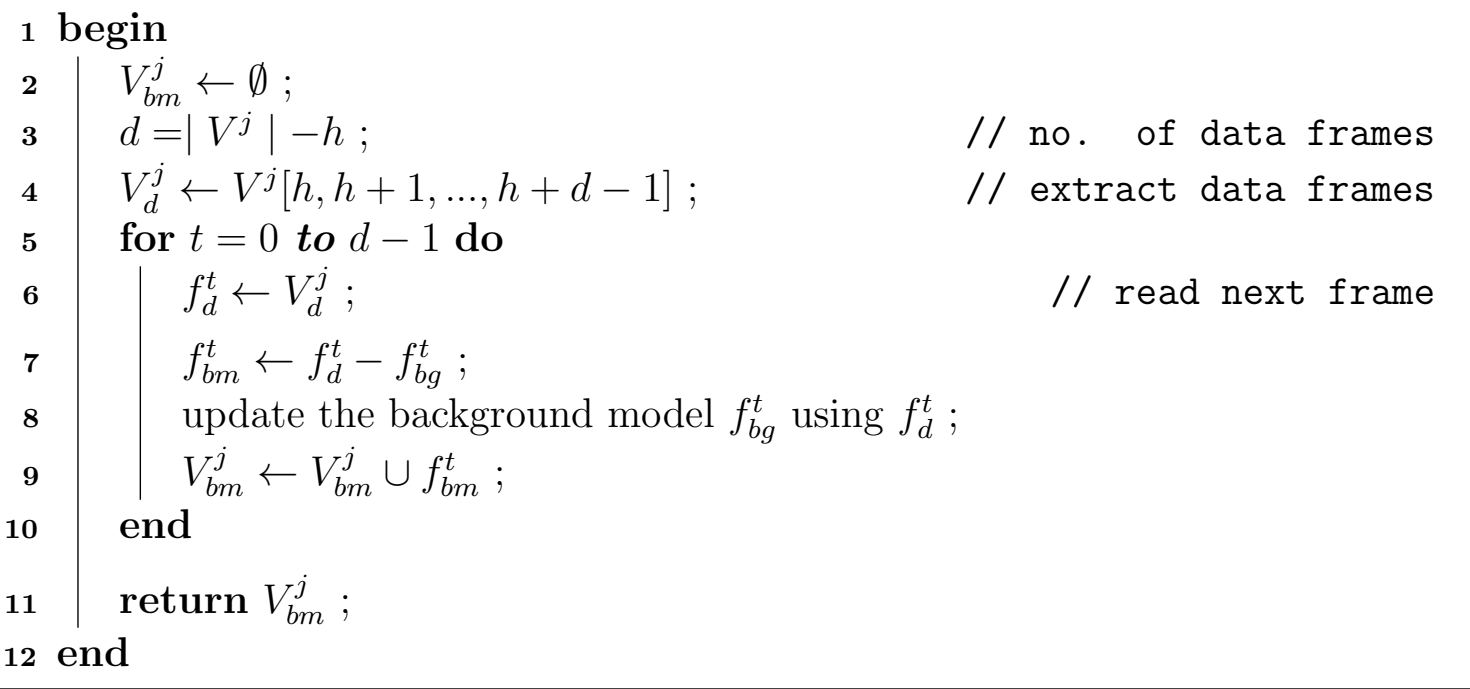

as black pixels, and the foreground pixels as white pixels; in other words, the white pixels refer to the change detection. Let $V_{h}^{j}$ denote the training set part of a video segment $j$, and $V_{d}^{j}$ denote the data frame part. At any time $t$, the binary mask frame $f_{b m}^{t}$ can be generated by subtracting $f^{t}$ from $f_{b g}^{t}$, where $f^{t}$ denotes the data frame, and $f_{b g}^{t}$ denotes the background model at time $t(0 \leq t<d)$.

The scheme that will be used for sampling is to consider all frames in the training set to generate the initial background model. However, while processing the data frames, the background model will be updated with every frame if a significant change has shown up in the scene; otherwise, only some frames (a sample) will be used to update the background model. This scheme can be achieved as follows. All frames $\left\{f_{h}^{i}: 0 \leq i<h\right\}$, belonging to the training set $V_{h}^{j}$, are processed to model the initial background $f_{b g}^{0}$. The next frame in the sequence will be the first frame in the data frames $V_{d}^{j}\left(f_{d}^{0}\right)$, and this frame will be subtracted from $f_{b g}^{0}$ to output $f_{b m}^{0}$. To reduce the computational cost, the background model will not be updated with every data 
frame. A double-threshold based technique is used to determine which frames will be participating in updating the background model. To do this, the data frames initially are partitioned into parts, and each part is referred to as a window of size $w_{d}$. At the beginning of each window, AoC in the current frame will be estimated. AoC is the proportion of white pixels in the resulting $f_{b m}^{t}$ at time $t$ (see Figure 4.1). It is a real number in the interval $[0,1]$, where the value ' 0 ' means there is no change, and the value ' 1 ' means all pixels have changed. If AoC is larger than a predefined high threshold $T_{h}$, all the data frames belonging to the current window will participate in updating the background model. On the other hand, if AoC is lower than a predefined low threshold $T_{l}$, every $i^{\text {th }}$ data frame in the current window takes part in updating the background model. If AoC is in the interval $\left[T_{l}, T_{h}\right]$, the algorithm remains in its current state. For instance, if the algorithm was in the sampling state, it continues with sampling data frames. However, if the algorithm was in a nonsampling state, it continues considering all the data frames in the current window to update the background model. For each subsequent window, the same procedure is applied iteratively until the last window. If the size of the last window is smaller than $w_{d}$, the algorithm will consider the sampling until the last data frame in the window (see Algorithm 5). Further details are included in Appendix A.3.

\subsection{Experiments and Evaluation}

This section describes two sets of experiments that were conducted to compare the proposed SdCD with SimpleCD (the MapReduce-based Change Detection algorithm without sampling). The first set of experiments is to show the accuracy of using SdCD compared to using SimpleCD. The second set of experiments compares the performance of the two approaches. 


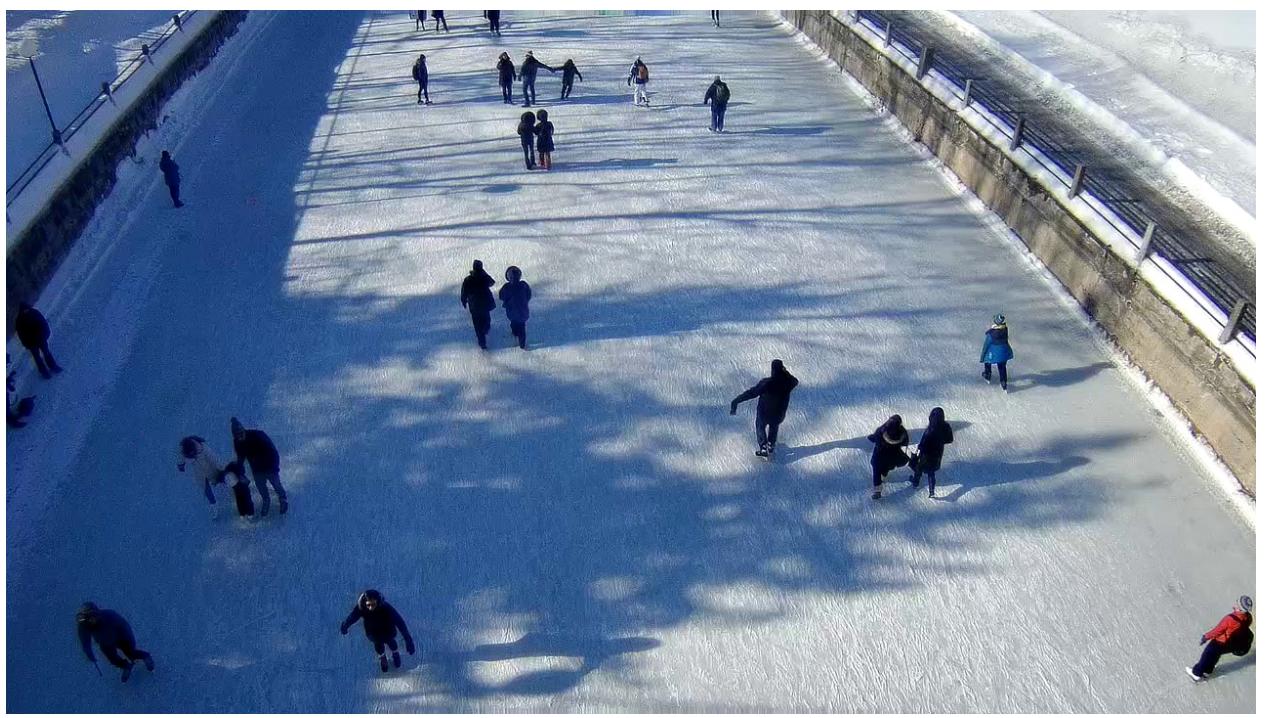

(a)

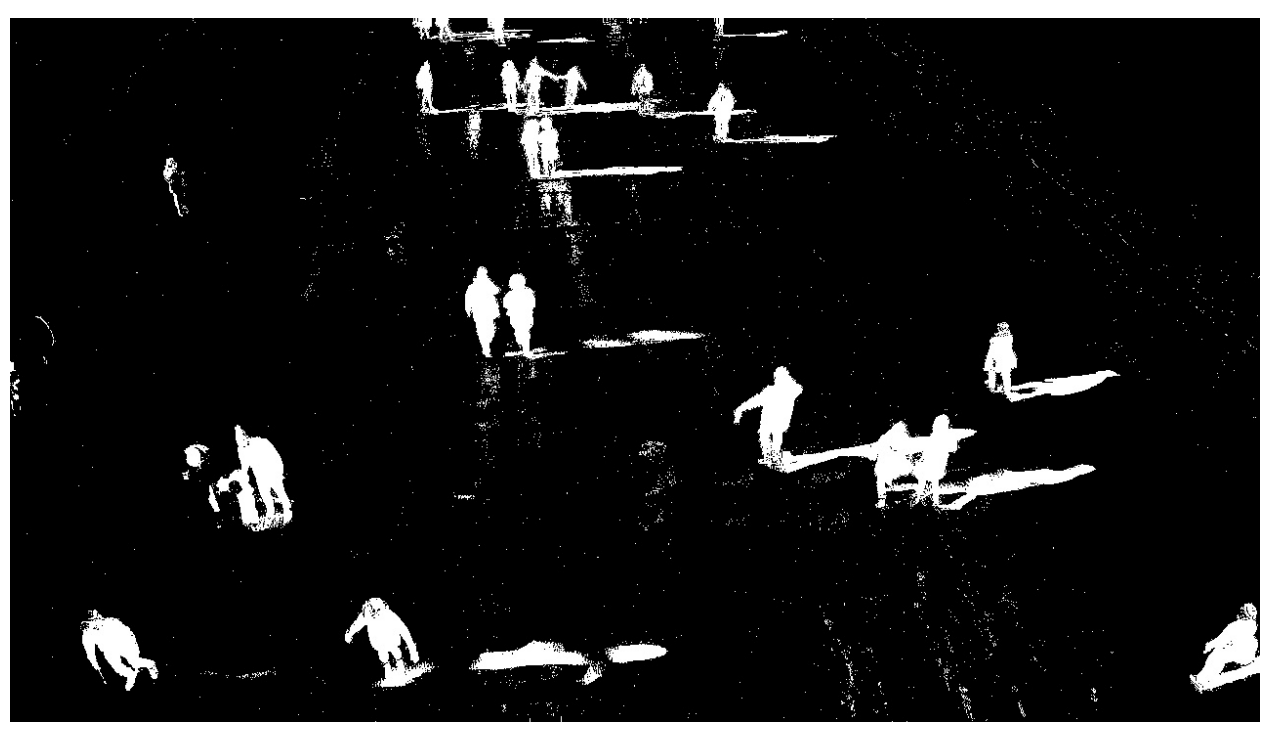

(b)

Figure 4.1: Displaying AoC in frame (a) as a binary mask frame (b). 


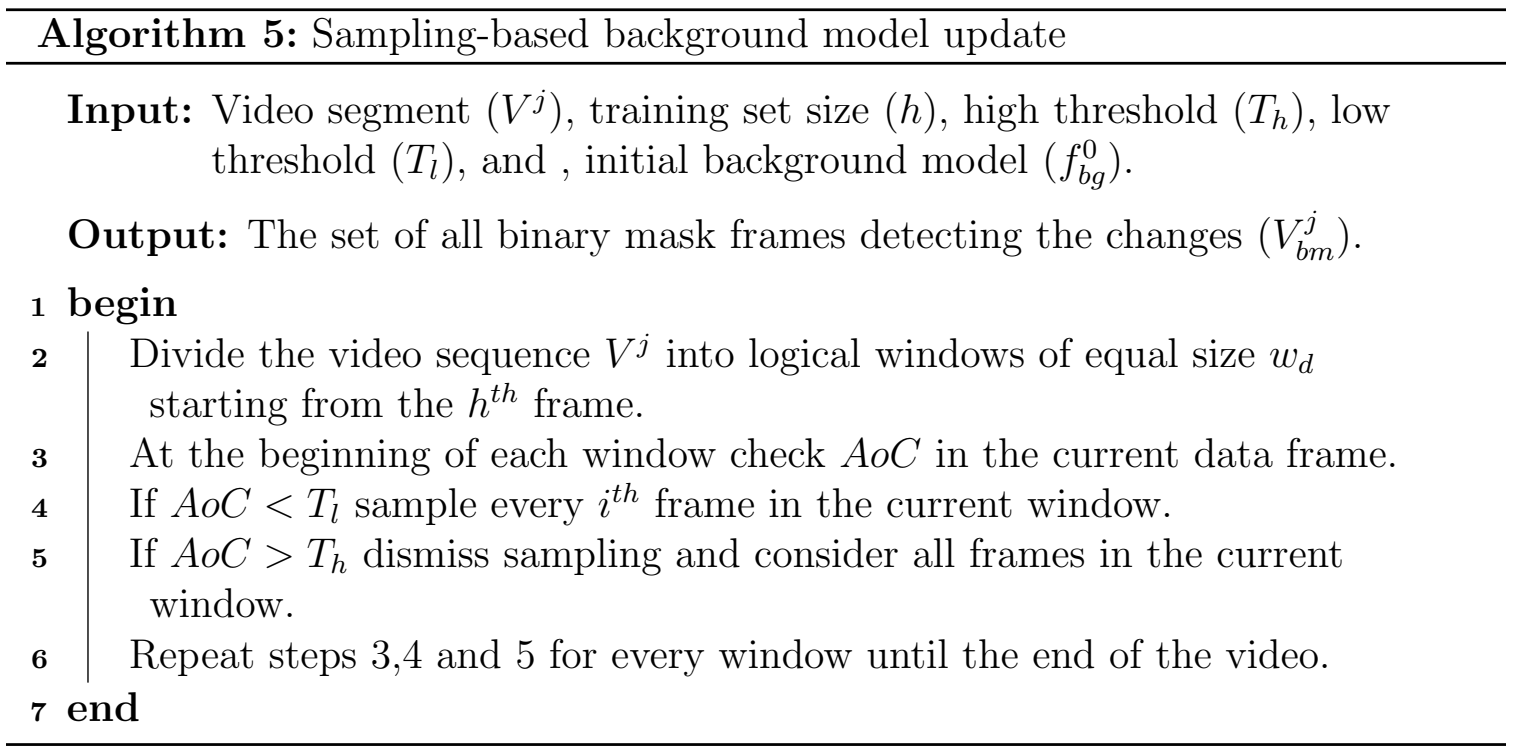

\subsubsection{Accuracy Evaluation}

To evaluate the accuracy of both SdCD and SimpleCD, the ROC curve will be used. The ROC curve plots the True Positive Rate (TPR) and False Positive Rate (FPR) pairs for different threshold values used to determine whether a pixel belongs to the foreground or the background. As described in Section 4.2.1, better accuracy can be achieved when the ROC curve approaches to the top-left corner; in other words, the value of TPR is '1', and the value of FPR is '0', which means that all foreground pixels are detected with no errors.

Hadoop has been designed to process big data, so the dataset used for both accuracy test and performance evaluation was recorded for skating people for 2 hours and 40 minutes. The recording resolution is $720 \mathrm{p}$ with $30 \mathrm{fps}$ (frame rate). The camera divided the recording into 32 files such that each one holds 5 minutes of recording (246MB file size); this means that the total dataset size is roughly 7.7GB. Since ground truth is required to perform the accuracy test, an annotation tool known as GNU Image Manipulation Program (GIMP) [93] is used to generate the ground truth for some randomly selected frames from the recording. The skating dataset will be 
used in the performance evaluation for that the size of the data is big enough to benefit from using Hadoop.

The ROC curve for the skating dataset (Figure 4.2) shows that the accuracy of SimpleCD is only marginally better than SdCD. However, this negligible loss in accuracy is accompanied by a significant improvement in the performance of SdCD (Section 4.2.2). SimpleCD uses all the frames in the sequence to update the background model, while SdCD uses some of the frames (sample frames) to reduce the computation of updating the background model. The sample frames are selected based on AoC in the scene as well as $T_{l}$ and $T_{h}$. The training set of size $(h)$ is required to model the initial background. The window size $(w)$ and the time lapse $\left(i^{t h}\right)$ values are fixed at 150 and 30 respectively to reduce the computation; the window size 150 means that $\mathrm{AoC}$ is computed once every 150 frames, and the time lapse 30 means that SdCD uses every $30^{\text {th }}$ frame for sampling within the current window. The high and low thresholds ( $T_{h}$ and $T_{l}$, respectively) are used to make the decision to update the background model. The two threshold values are experimentally determined in such a way that we do not significantly degrade the accuracy of CD. Testing the accuracy requires human intervention to generate the ground truth. Based on the experimental accuracy evaluation, if AoC is larger than '0.03', every frame in the current window is used to update the background model. However, if AoC is smaller than '0.015', every $30^{\text {th }}$ frame in the current window is used to update the background model. It is important to note that specifying these two parameters differs from a scenario to another depending on the content of the video recording.

To specify the values of the two thresholds, two recordings need to be used as follows: The first recording includes a scene with low people density, and the second recording includes high-density people. The assumption here is that a priori knowledge about the scene is essential in this case to set the threshold values. In other 


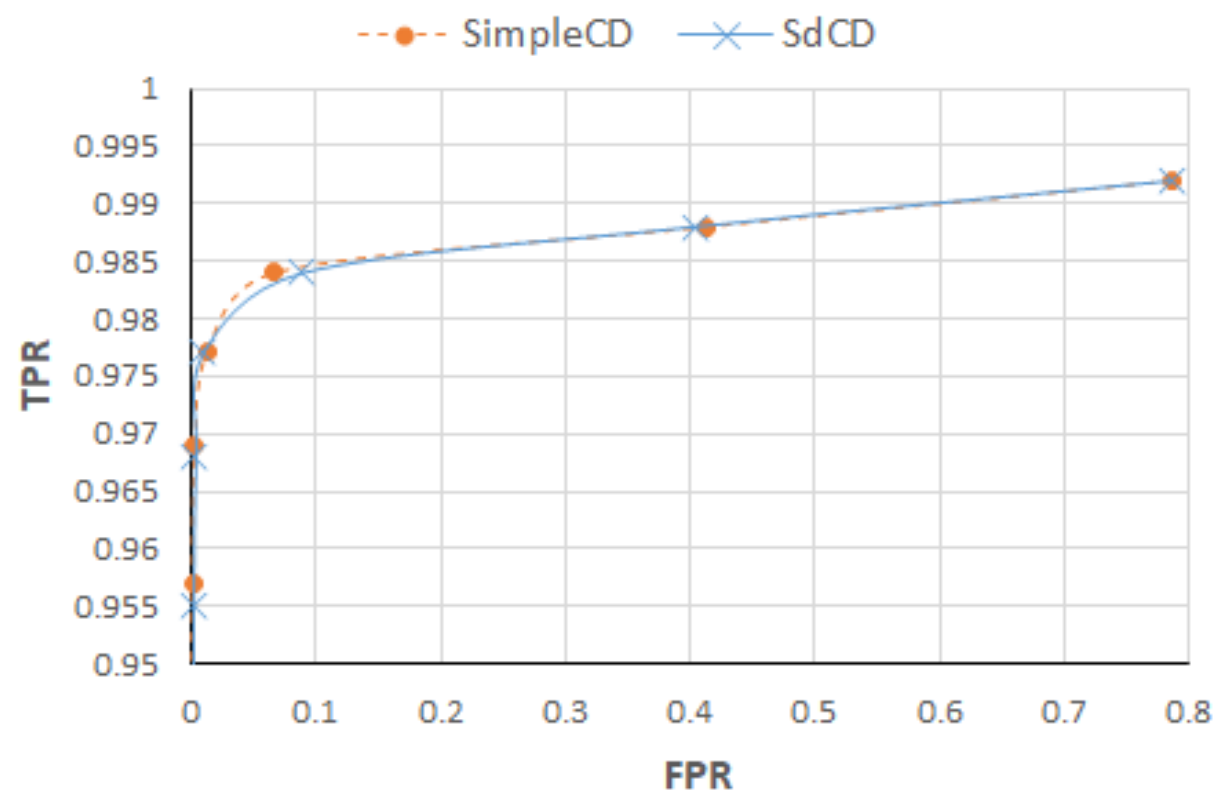

Figure 4.2: Accuracy test for the skating dataset.

words, the high and low thresholds represent the way by which the algorithm differentiates between the high and low-density scene. In the realm of computer vision, human judgment is considered the ground truth (or the reference to evaluate the accuracy). This means that the two thresholds are specified based on the experiments and human judgment. Fortunately, it is unnecessary to watch all recordings to make this judgment; however, two short recordings (e.g., two minutes long) are sufficient. One minute taken from a crowded scene can be used to estimate the high threshold value, and another minute taken from a not crowded scene is used to estimate the low threshold value. The low threshold is estimated by calculating the AoC for all the frames of the first recording and considering the highest value. On the other hand, the high threshold can be estimated by considering the smallest $\mathrm{AoC}$ value of the second recording.

Figure 4.3 shows an example to demonstrate how the estimated threshold values make the decision to use sampling or not while updating the background model. The 


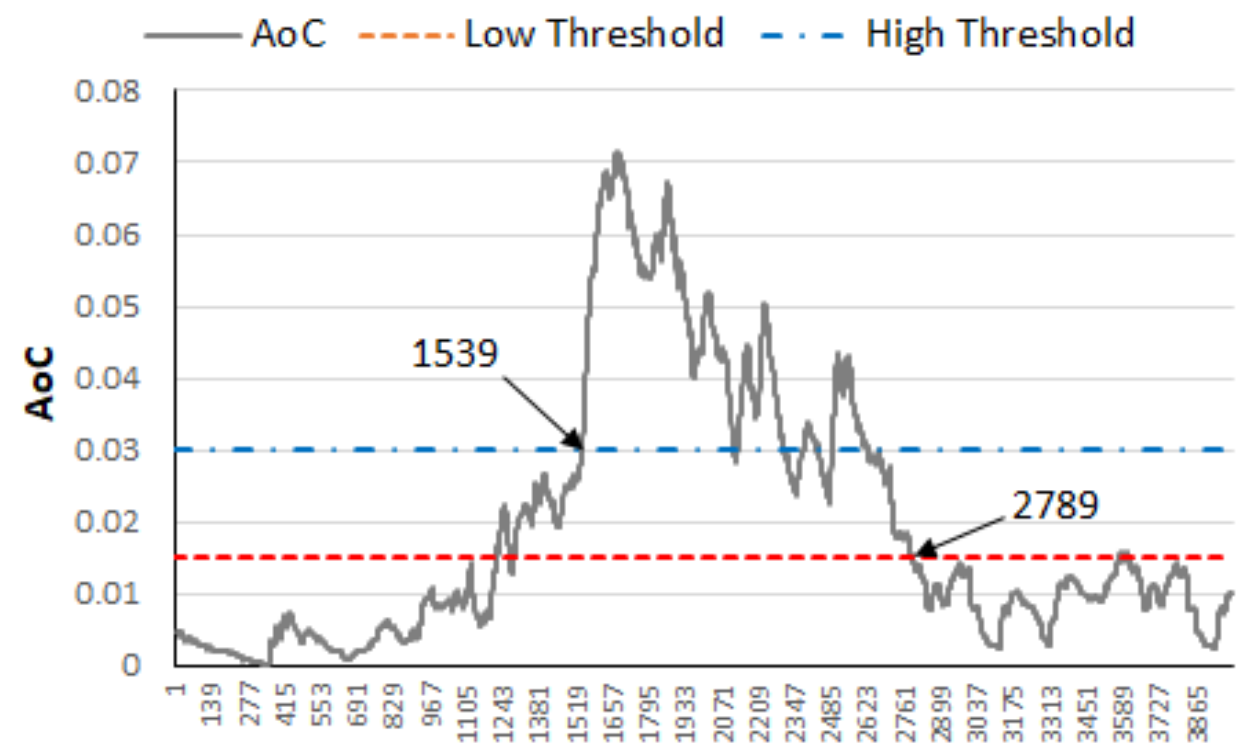

Frame sequence number

Figure 4.3: Example of how the threshold values ( $\mathrm{Tl}$ and $\mathrm{Th}$ ) decide sampling.

$\mathrm{x}$-axis represents the frame numbers (the offset of each frame from the beginning of the sequence), and the $\mathrm{y}$-axis represents $\mathrm{AoC}$ in the frames. The low threshold $T_{l}$ and the high threshold $T_{h}$ are depicted as dashed and dash-dotted horizontal lines, respectively. It can be observed that it is not until frame number 1539 that SdCD is using every frame (no sampling) to update the background model before it returns back to sample the frames after frame number 2789. In other words, SdCD samples the frames to update the background model from the beginning until the first window check after frame 1539. Recall that during the execution of SdCD, AoC is calculated at the beginning of each window. SdCD does not perform sampling between frames 1539 and 2789, but after the first window check is performed after frame 2789, it returns to sampling the frames again, as detailed in Algorithm 5. 


\subsubsection{Performance Evaluation}

To evaluate the performance of the proposed SdCD technique, a prototype of the algorithm was implemented and compared with a prototype implementation of the SimpleCD technique. The performance metrics used in this evaluation include execution time, speedup, and relative speedup. The execution time in this context is the time required by a MapReduce job to complete all of its tasks on all nodes. The speedup is defined as the time of running sequential CD divided by the time of parallel CD (SimpleCD) or sampling-based parallel CD (SdCD) on a Hadoop cluster.

\subsubsection{Technologies and Cluster Setup}

The open-source library for computer vision applications, OpenCV, was used to facilitate processing video data. MapReduce-based Java applications can interface to OpenCV native code, designed for $\mathrm{C} / \mathrm{C}++$ native applications, by using JNI. Cloud computing technology was used to provide the required hardware resources. Amazon EC2 instances [94] were used in the experiments to establish a 17-node Hadoop cluster on the cloud. Each node (t3.xlarge) in the cluster consisted of 4 vCPUs $2.5 \mathrm{GHz}$ Intel Scalable Processor (64-bit), and 16 GB RAM. Each node also ran Ubuntu Linux OS version 18.04 LTS, Java Virtual Machine [95], and Hadoop's daemons (Hadoop 2.8.5). One node was configured as a master to run the NameNode, Secondary NameNode, ResourceManager, and the Hadoop daemons. The rest of the nodes were configured as workers such that each node was prepared to run two Hadoop daemons: a NodeManager and a DataNode. It is important to know that the video files were distributed evenly among the nodes to be processed in parallel. For instance, when the degree of parallelism is 16 , the 32 video files are distributed such that each node stores 2 files to balance the load. To process video data distributed on $p$ nodes, each node hosts only one map task. The experiments had been repeated three times, at 
Table 4.1: SdCD and SimpleCD execution time (seconds) comparison.

\begin{tabular}{|c|c|c|c|c|}
\hline \multirow{2}{*}{ Algorithm } & \multicolumn{4}{|c|}{ Execution time (sec.) using different number of nodes } \\
\hline & 1 & 4 & 8 & 16 \\
\hline SimpleCD & 7136 & 1858 & 939 & 492 \\
\hline SdCD & 4696 & 1246 & 633 & 337 \\
\hline
\end{tabular}

least, and the measurements were within $\pm 5 \%$ of the average.

\subsubsection{Results of Performance Evaluation}

The experimental results show that the execution time of processing the dataset on one node was 7136 seconds, this time decreased to 492 seconds when the same dataset was processed in parallel using SimpleCD on 16 nodes. On the other hand, when SdCD was used to process the dataset, the execution time decreased to 337 seconds using the 16 nodes. This means that SimpleCD achieves a speedup of up to 14.5, while SdCD attains a speedup of $21.1 x$. The speedup improvement achieved by using SdCD is because of the proposed sampling method.

To analyze the performance of the two algorithms as the number of nodes increases, a set of experiments to process the video data on 1, 4, 8, and 16 nodes was conducted. Figure 4.4 shows the execution time improvement of using SdCD compared to SimpleCD. The x-axis depicts the number of nodes (the degree of parallelism), and the y-axis depicts the execution time in seconds. The execution time of both SdCD and SimpleCD decreases as the number of nodes increases, but SdCD achieves better performance, as shown in Table 4.1.

Figure 4.5 shows the effect of the sampling method (used in SdCD) on reducing the execution time as the degree of parallelism increases. The $\mathrm{x}$-axis represents the number of nodes, and the y-axis represents the execution time improvement of SdCD relative to SimpleCD because of the sampling effect. With an increase in the degree of 


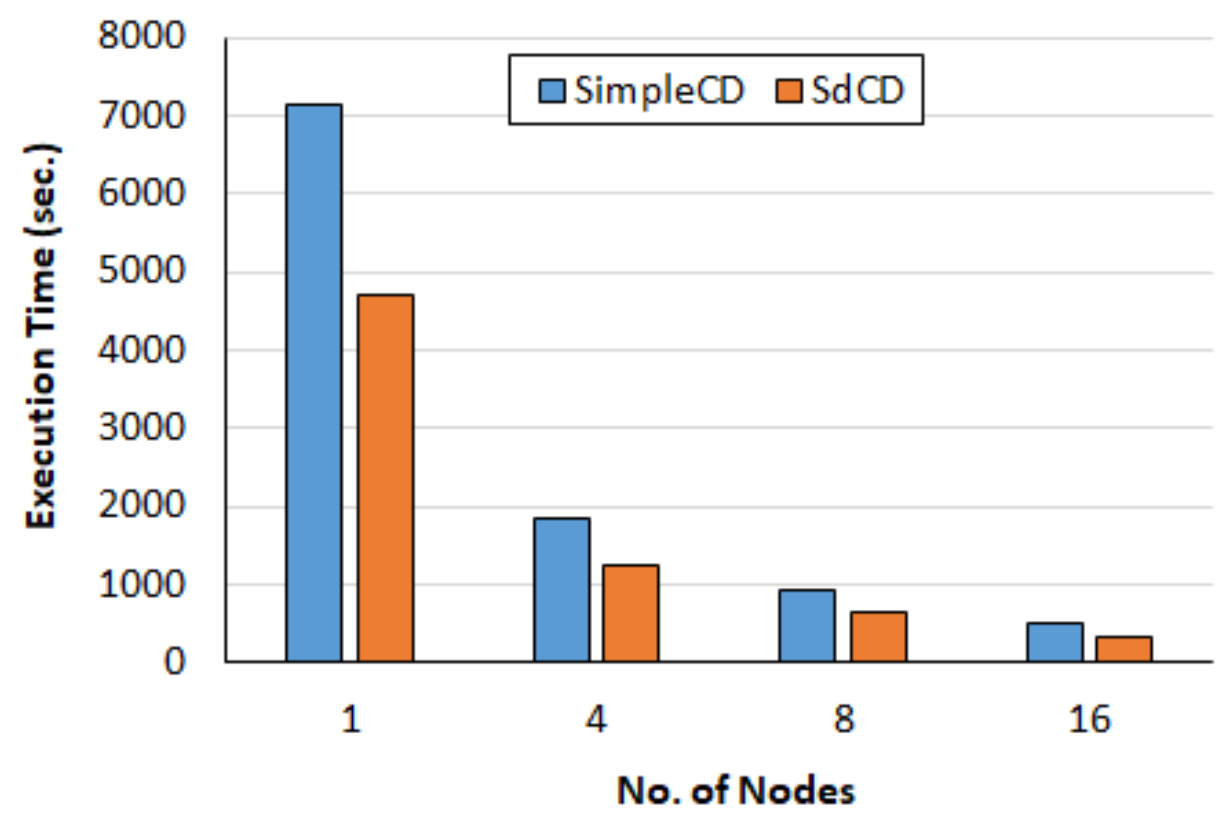

Figure 4.4: Execution time as the number of nodes increases.

parallelism, the improvement coming from the sampling effect marginally decreases. For instance, when 8 nodes were used, the execution time decreased from 939 seconds to 633 seconds (Table 4.1); i.e., SdCD reduces the execution time to $67.4 \%$ in comparison with SimpleCD (Figure 4.5). However, SdCD improves the execution time to $68.5 \%$ of SimpleCD when 16 nodes were used. This is because when a job is divided among more nodes, each node will process less data, so the job is becoming more susceptible to the overhead of Hadoop. Because of sampling, SdCD reduces the computation on each node, so it becomes more influenced by the overhead of Hadoop. Recall that small running jobs are more influenced by Hadoop overhead. However, by processing larger data, the overhead impact is mitigated. Dividing a job into smaller subtasks running in parallel makes the job execution time more sensitive to slow-running tasks [14]. One slow-running task results in longer execution time for the entire job, and by increasing the level of parallelism, the job becomes more dependent on the effect of slow-running tasks. 


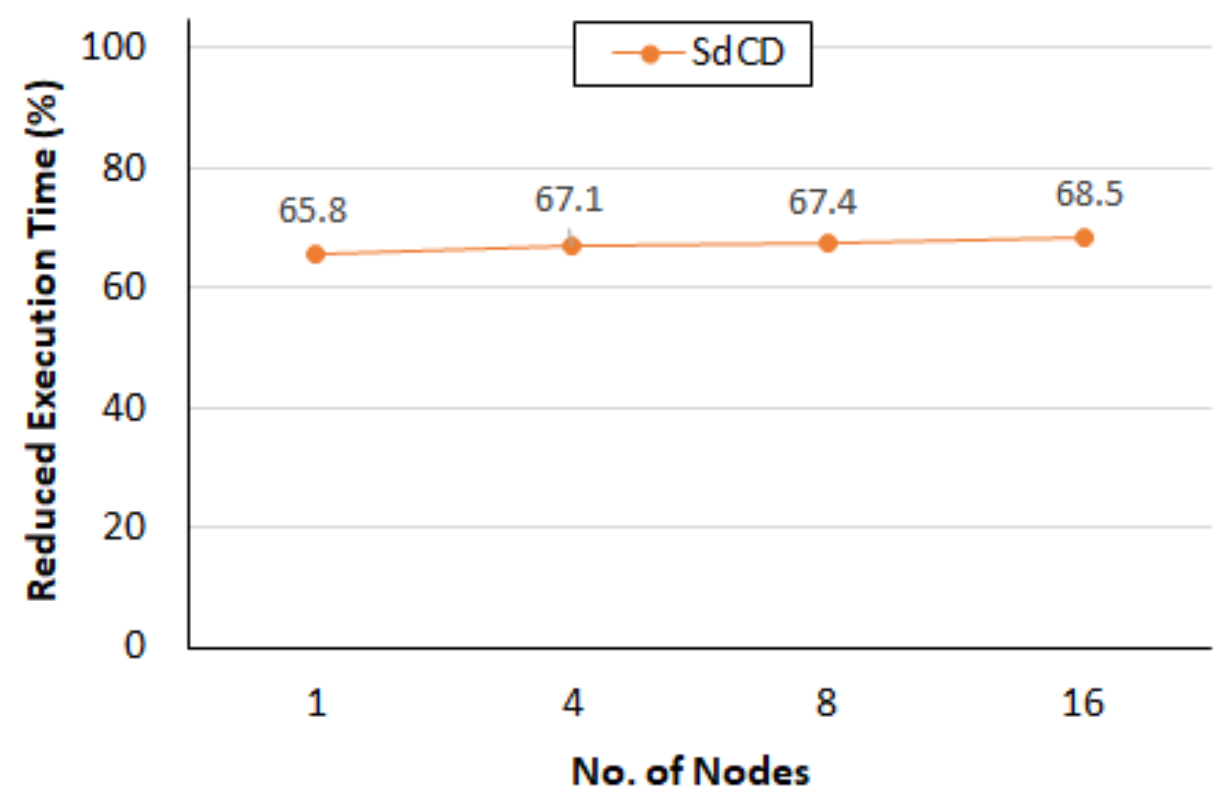

Figure 4.5: The execution time improvement achieved by SdCD.

Figure 4.6 shows the speedup for both SdCD and SimpleCD to analyze performance as the number of nodes increases. The x-axis refers to the number of nodes, and the $y$-axis refers to the achieved speedup. The figure reveals that the speedup of SimpleCD is slightly better than SdCD as the number of nodes increases. For example, SimpleCD achieves a speedup of 7.6 on 8 nodes, while SdCD achieves a speedup of 7.4. As the number of nodes increases to 16, SimpleCD attains a speedup of 14.5, whereas SdCD attains a speedup of 13.9. This is because the amount of computation is larger when SimpleCD has been used. From another perspective, SdCD can process larger data to achieve speedup equivalent to SimpleCD.

\subsection{Chapter Summary}

Change detection is an important method that can be used to avoid unnecessary computation in many video processing applications. This thesis proposes a change detection approach to improve the performance of processing big video data stored in 


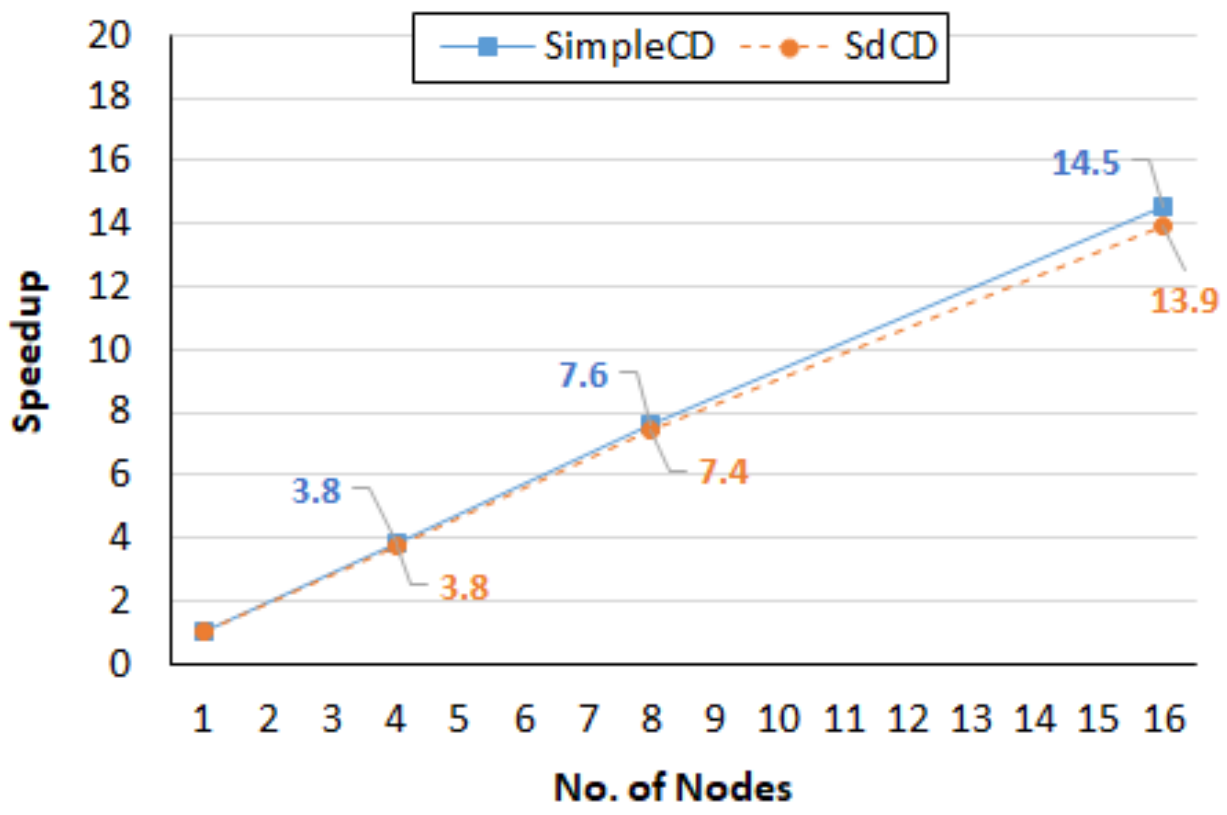

Figure 4.6: Speedup.

Hadoop clusters in a timely manner. This approach relies on using the MapReduce programming model to process the video data in parallel and using a sampling method to reduce the computation on each node. The performance of the proposed approach was studied empirically, and the experimental results show how the solution reduces the execution time without significantly sacrificing accuracy. 


\section{Chapter 5}

\section{Change Detection on Fault-Tolerant Systems}

The goal of this chapter is to demonstrate the effect of various sampling techniques to improve the performance of processing big video data in Hadoop clusters. This chapter discusses the effect of sampling on reducing the overhead accompanied by overlapping some video frames and avoiding unnecessary computation in unreliable systems. In this chapter, there are three sampling algorithms as follows: sampling history frames, sampling data frames, and sampling both history frames and data frames algorithms. This chapter investigates the effect of the previous sampling algorithms on the execution time in the normal case and in the case of a node failure.

\subsection{Overhead of MapReduce-Based Change De- tection}

Running MapReduce-based CD on a Hadoop cluster experiences overhead that affects the parallel execution time. The parallel execution time can be modeled:

$$
T_{p}=\frac{C}{p}+O
$$


where $T_{p}$ refers to the parallel execution time, $C$ refers to the computation time spent to solve the problem, and $p$ refers to the degree of parallelism. The term $O$ refers to the overhead.

There are several sources of overhead in Hadoop, such as the management overhead, load imbalance, slow running tasks that dominate the entire job execution time, and extra computation. In the rest of this chapter, $O$ will refer to the overhead coming from the extra computation resulted in by processing the overlapping frames twice. The goal of this thesis is to reduce change detection computation time $C$, and to minimize the overhead $O$. To achieve this, two different sampling techniques have been devised to improve the performance of MapReduce-based CD running on Hadoop clusters.

\subsubsection{MapReduce-based CD Problem}

In sequential CD, all the frames in the entire video data participate in the computation of the background model. The first $h$ frames (history training set) are used to estimate the initial background model, while the remaining $m$ frames (data frames) are used to update the background model. The data frames are also subtracted from the background model to generate the output as binary mask frames. The execution time of the sequential $\mathrm{CD}$ is $\Theta(h+m)$.

Video data $\mathrm{V}$ should be partitioned into s segments such that $V_{j}: 0 \leq j<s$; hence the segments can be distributed across the nodes on the Hadoop cluster. For modeling simplicity, assume the $\mathrm{m}$ data frames of $V$ can be evenly divided among the $s$ video segments so that each segment holds $\mathrm{m} / \mathrm{s}$ data frames. If the number of frames is not a multiple of the number of segments, the last segment will hold fewer frames, and the execution time will be affected by the other segments. In other words, all segments except the last one will hold $\lceil\mathrm{m} / \mathrm{s}\rceil$ frames, but the last segment will hold $m-\lceil m / s\rceil *(s-1)$. To detect the changes in video segment $j\left(V^{j}\right)$, an 
initial background model $\left(f_{b g}^{0}\right)$ needs to be estimated from the history training set, $f^{0}, f^{1}, \ldots, f^{(h-1)}$, of size $h$ at the beginning of the segment. Let $V_{h}^{j}$ denote the history training set (or history frames) of video segment $j\left(V^{j}\right)$. The history frames, $V_{h}^{j}$, for each segment $(0 \leq j<s)$ are overlapped with the previous segment $\left(V^{j-1}\right)$; however, the history frames of the first segment $\left(V_{h}^{1}\right)$ are not overlapped. The remaining frames of $V^{j}$ represent the data frames, $\left\{f^{0}, f^{1}, \ldots, f^{m / s-1}\right\}$ of size $m / s$, from which the changes in the scene are detected. Let $V_{d}^{j}$ denote the set of data frames of $V^{j}$. For each data frame $f_{d}^{t}$ at time $t(0 \leq t<m / s)$, the changes are detected as a binary mask frame $f_{b m}^{t}$ at the same time. Let $V_{b m}^{j}$ denote the set of all binary mask frames for $V^{j}$ (the output of CD).

In the simple MapReduce CD approach, the video segments $\left\{V^{0}, V^{2}, \ldots, V^{s-1}\right\}$ are processed on $p$ Hadoop nodes. The parallel computation includes $s$ segments, and each segment consists of $h$ history frames and $\mathrm{m} / \mathrm{s}$ data frames. The execution time of SimpleCD per video segment is $\Theta(h+m / s)$. Since the number of segments may be larger than the degree of parallelism $(s \leq p)$, each Hadoop node could process more than one segment. Let the maximum number of segments to be processed on one node is $k$ such that $k=\lceil s / p\rceil$. Hence, the parallel execution time of SimpleCD will be $\Theta(k(h+m / s))$. The goal of this thesis is to use two sampling techniques along with MapReduce to improve the execution time of CD. Algorithm 6 shows the details of this approach.

\subsubsection{The Sampling of History Frames Based Approach}

In SimpleCD, processing the overlapping frames (history training set) of all video segments except the first segment, $\left\{V_{h}^{j}: 0 \leq j<s\right\}$, is considered extra computation overhead $O$ (see equation 5.1). To minimize the overhead, $h^{\prime}$ frames will be sampled out of $h$ history frames of each segment to be considered in the computation. This approach, called ShCD, uses MapReduce-based CD for parallel processing and sampling 


\section{Algorithm 6: SimpleCD}

Input: Video segment $\left(V^{j}\right)$, training set size $(h)$, initial background model $\left(f_{b g}^{0}\right)$.

Output: The set of all binary mask frames detecting the changes $\left(V_{b m}^{j}\right)$.

\section{1 begin}

$2 \quad$ Run Algorithm 3 to generate the initial background model;

$3 \quad$ Run Algorithm 4 to update the background model;

4 end

the history training set for minimizing the overhead $O$. For each video segment $V^{j}$, ShCD will process only $h^{\prime}$ sample history frames to estimate the initial background model $\left(f_{b g}^{0}\right)$, and $\mathrm{m} / \mathrm{s}$ data frames to update the background model. Therefore, the execution time per video segment is reduced to $\Theta\left(h^{\prime}+m / s\right)$. Since each Hadoop node may process more than one segment ( $k$ segment at most), the parallel execution time for ShCD will be $\Theta\left(k\left(h^{\prime}+m / s\right)\right)$.

The idea of this sampling technique is to slide a window of size $w_{h}$ over the history training set $\left(V_{h}^{j}\right)$ of segment $j$ to sample some frames that will be used in estimating $f_{b g}^{0}$ for the segment. The window, called History Sampling Window (HSW), is an array that holds the value '1' at the indices that match the Fibonacci sequence $\{1,2,3,5,8, \ldots\}$, and the value '0' otherwise. During sliding HSW, ShCD considers the frames that match the value ' 1 ' in the window in estimating the initial background model $\left(f_{b g}^{0}\right)$ and ignores the frames that match the value '0'. Figure 3.2 shows an example of sliding HSW of size 8 over a history training set of size 16, as described in Section 3.1.2. The difference here is that this technique is used to consider some frames during processing, while it was used in Chapter 3 in partitioning large video files.

Algorithm 7 describes sampling the history frames in some details. In line 3, HSW is initiated by assigning the value ' 1 ' to the indices that match the Fibonacci sequence 


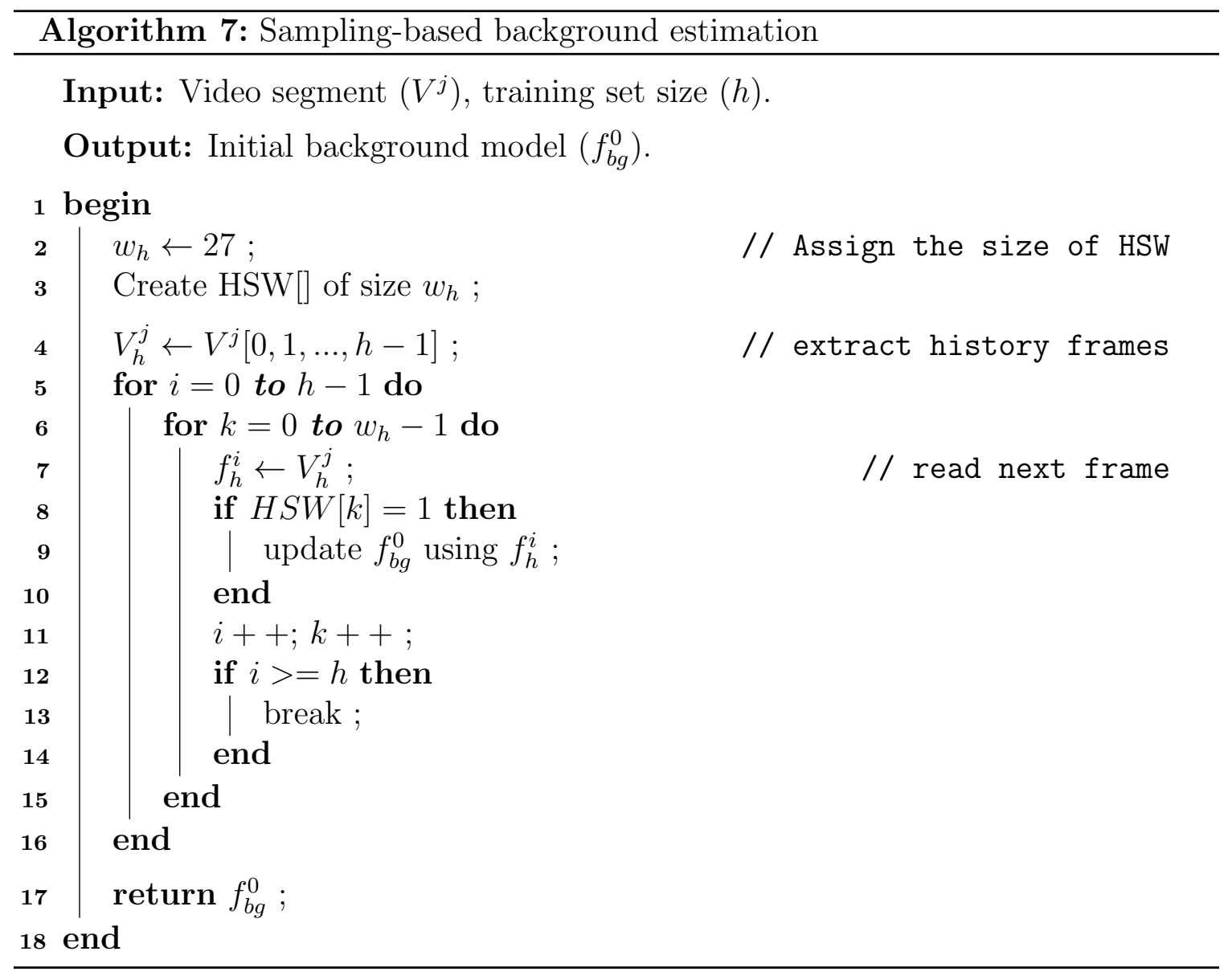

and the value ' 0 ' otherwise. The loop in line 4 is to iterate over the history frames, and the inner loop in line 5 is to slide HSW to decide which frames will be considered in estimating the initial background model $\left(f_{b g}^{0}\right)$. The two parts of ShCD (estimating and updating the background model) are shown in Algorithm 8.

\subsubsection{The Sampling of Data Frames Based Approach}

This method, called SdCD, depends on using MapReduce for parallel processing and a sampling technique for reducing the problem computation time $\mathrm{C}$ (see equation 5.1). The proposed sampling technique is based on the double thresholding method to avoid unnecessary computation by deciding whether to consider every data frame in updating the background model. SdCD uses the entire history frames $\left(V_{h}^{j}\right)$ of 


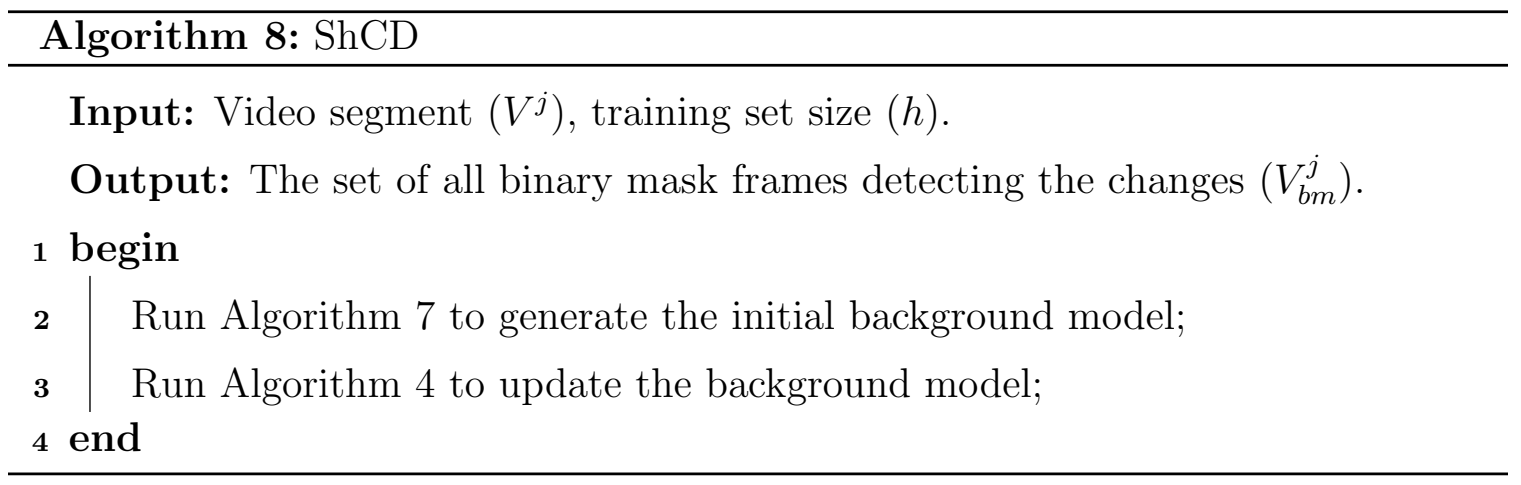

segment $j$ to estimate the initial background model $\left(f_{b g}^{0}\right)$ for the segment.

According to SdCD, $V_{d}^{j}$ is divided into logical parts called Data Sampling Window (DSW) of size $w_{d}$. At the beginning of each window, AoC in the current data frame $\left(f_{d}^{t}\right.$ at time $t$ ) will be calculated. AoC is the proportion of white pixels in $f_{b m}^{t}$ at time $t$ (review Section 4.1.3). AoC takes a real number in the interval $[0,1]$, where the value '0' means that there is no change, and the value '1' means that all pixels have changed. If AoC is larger than a predefined high threshold $T_{h}$, all the data frames belonging to the current DSW will be used to update the background model $\left(f_{b g}^{t}\right.$ at time $t$ ). However, if AoC is lower than a predefined low threshold $T_{l}$, every ith data frame in the current DSW is used to update the background model. The same steps will be repeated for all the subsequent DSW until the end of the video segment. The details of this sampling technique are described in Chapter 4, Algorithm 5.

The example in Figure 5.1 illustrates how the proposed data sampling technique works. The figure displays two consecutive data sampling windows of size 9: $D S W_{i}$ and $D S W_{i+1}$. At the beginning of $D S W_{i}$, the algorithm checks AoC calculated from the binary mask frame $f_{b m}$ for the first data frame. Since AoC is low, the algorithm samples every $3^{\text {rd }}$ frame, and then only the sample frames are considered in updating the background model. In $D S W_{i+1}$, the technique considers all the frames because the AoC at the first frame is high.

According to this technique, all $h$ history frames of video segment $j\left(V_{h}^{j}\right)$ will 


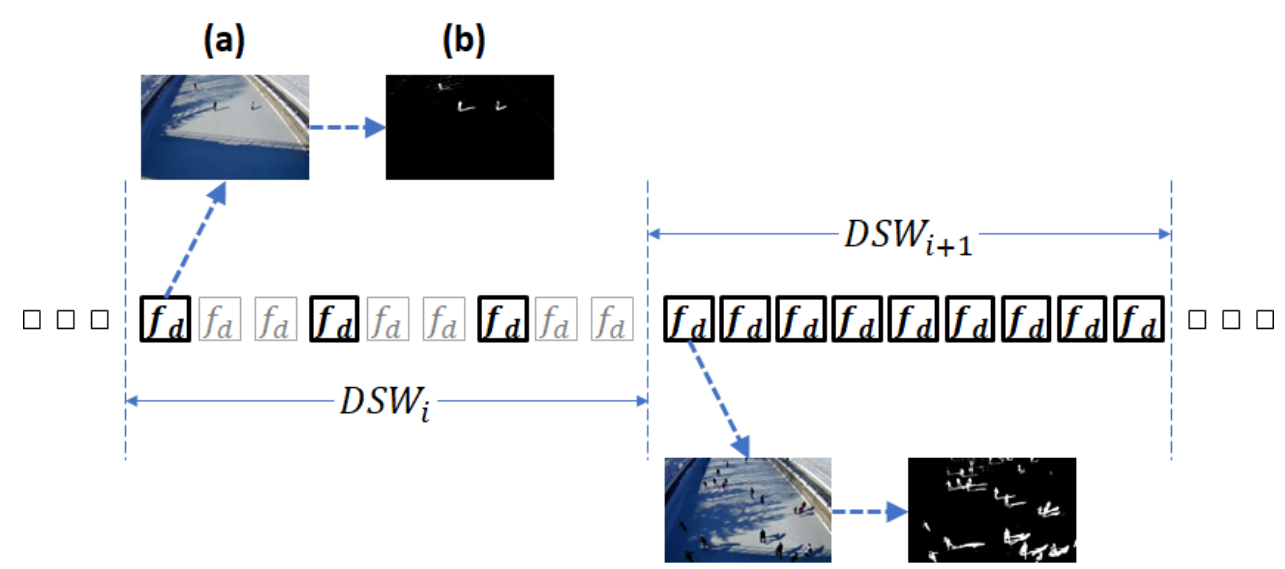

(c)

(d)

Figure 5.1: Data frames sampling technique.

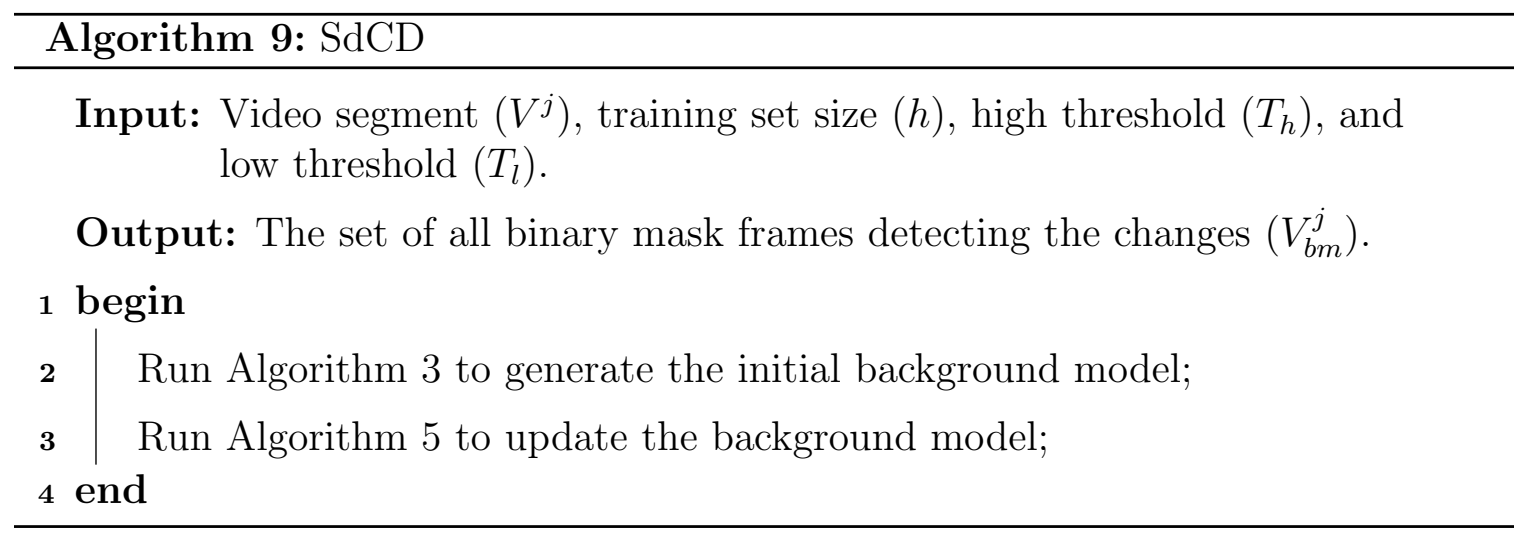

be used to estimate $f_{b g}^{0}$; however, $(\mathrm{m} / \mathrm{s})^{\prime}$ data frames are sampled out of $\mathrm{m} / \mathrm{s}$ to update the background model for the video segment. Therefore, the execution time of SdCD per video segment is $\Theta\left(h+(m / s)^{\prime}\right)$, and the parallel execution time will be $\Theta\left(k\left(h+(m / s)^{\prime}\right)\right)$. This approach is illustrated in Algorithm 9.

\subsubsection{The Sampling of History and Data Frames Based Ap- proach}

The two sampling techniques (history and data) can be used together to reduce more computation. The resulting approach is called $S h d C D$. It uses MapReduce for 
parallel processing, sampling the history frames to minimize the extra computation overhead $O$ (Algorithm 7), and sampling the data frames to reduce the computation time $C$ (Algorithm 5, Chapter 4). In total, the execution time of ShdCD per video segment can be reduced to $\Theta\left(h^{\prime}+(\mathrm{m} / \mathrm{s})^{\prime}\right)$, and as a result, the parallel execution time reduces to $\Theta\left(k\left(h^{\prime}+(m / s)^{\prime}\right)\right)$. This approach is illustrated in Algorithm 10.

\subsection{Experiments and Evaluation}

To compare the performance of the MapReduce-based CD algorithms (SimpleCD, ShCD, SdCD and ShdCD) empirically, proof of concept prototypes have been developed. SimpleCD was used as a reference to evaluate the performance improvement achieved by the other three sampling-based approaches. There were three experiments conducted to study (1) the effect of increasing the degree of parallelism on system performance, (2) the impact of the video segment size on the execution time, and (3) the effect of a node failure on the execution time. To evaluate system performance, three metrics have been used: the execution time, parallel speedup $\left(S_{p}\right)$, and efficiency $\left(E_{p}\right)$ (see Section 3.2.2). To measure the performance of the proposed algorithms that depend on using both parallel model and sampling, a speedup metric (S) can be defined as follows. The speedup (S) equals the serial execution time of running CD without sampling divided by the parallel execution time of CD with sampling.

\subsubsection{Datasets}

The dataset used in this study was recorded from a real scene for people skating on a frozen canal. There are two scenarios recorded as follows: weekday and weekend. The weekday scenario was recorded during a weekday, where the scene was not crowded with people (several files). The weekend scenario includes recording (several files) 


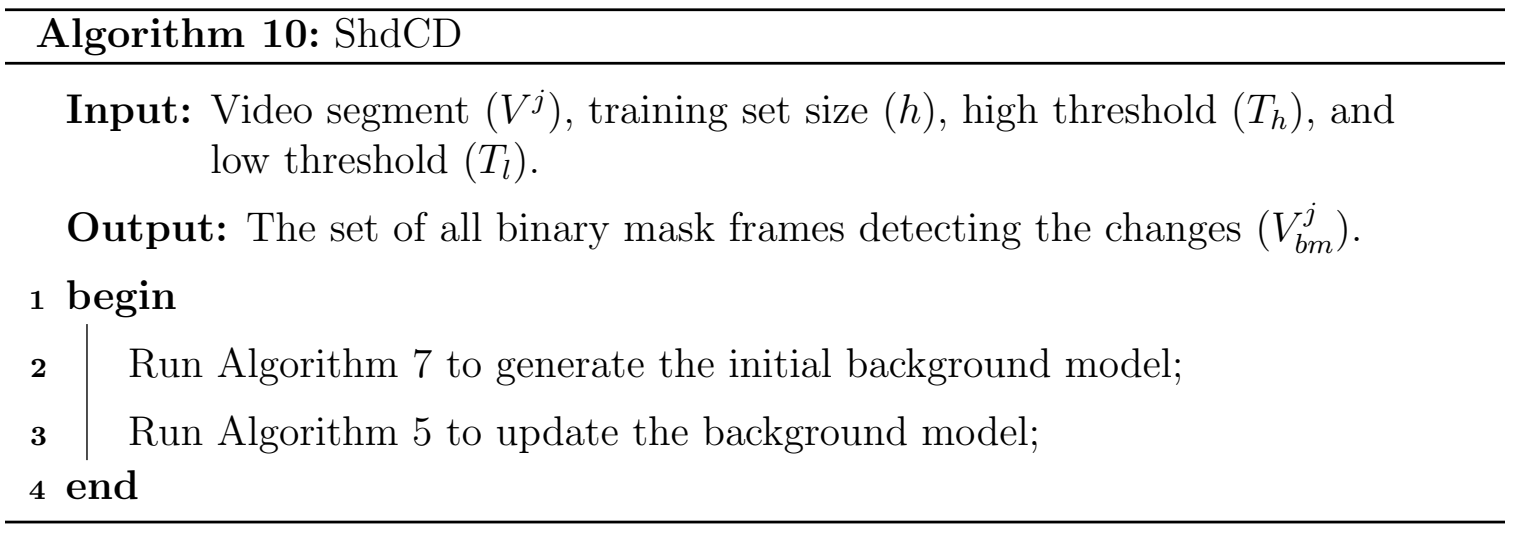

taken from the scene at the weekend, where the scene was crowded with people in $50 \%$ of the recording. The recording resolution is $720 \mathrm{p}$ with $30 \mathrm{fps}$ (frame rate), and the data size is approximately 8 GB. Each scenario is partitioned in three different ways: (1) a set of video segments where each segment includes 2.5 minutes of the recording (segment size is about $128 \mathrm{MB}$ ), (2) a set of video segments where each segment includes 5 minutes of the recording (segment size is about $256 \mathrm{MB}$ ), and (3) a set of video segments where each segment includes 10 minutes of the recording (segment size is about $512 \mathrm{MB}$ ). Each segment includes its own history training set, so it can be processed independently in any node in a Hadoop cluster.

\subsubsection{Technologies and Cluster Setup}

To process video data, OpenCV (open-source library for computer vision) was used. Cloud computing services such as Amazon Web Service (AWS) was used to provide the required hardware resources. The experiments were conducted on a 9-node Hadoop cluster using Amazon EC2 instances. Each instance (t3.xlarge) consisted of 4 vCPUs up to $2.5 \mathrm{GHz}$ Intel Scalable Processor (64-bit), and 16 GiB RAM. The network performance was up to 5 Gbps. Each node also ran Ubuntu Linux OS (version 18.04 LTS (HVM), 64-bit (x86)), Java VM (OpenJDK version 10.0.2), and Hadoop 
daemons (Hadoop 2.8.5). One node was configured as a master to run the NameNode, Secondary NameNode, ResourceManager Hadoop daemons. The rest of the nodes were configured as workers, where each node was prepared to run two Hadoop daemons: a NodeManager and DataNode. The HDFS block was set in such a way that the video segments do not split. Video segments were evenly distributed among the nodes involved in the parallel computing, and there was only one replica for each video segment unless stated otherwise. The experiments have been repeated three times, at least, and the measurements were within $\pm 5 \%$ of the average.

\subsubsection{MapReduce CD and Implementation details}

To support video processing, InputFormat and RecordReader have been extended using java classes named VideoInputFormat and VideoRecordReader, respectively, as mentioned in Section 3.1.3. Video data can be accessed in parallel using map tasks running on different DataNodes, where each video segment is assigned to one map task. As discussed in Chapter 4, the prototypes of the MapReduce-based CD approaches are based on a map-only job, and there is no need for the reduce tasks nor storing the output in HDFS.

The parameters for the sampling-based algorithms were set as follows. In Algorithm 7, when HSW of size 610 slides over a training set of size $900(h)$, it generates a sample training set of size $27\left(h^{\prime}\right)$. For more insight about determining the value of this parameter, the readers are referred to Chapter 3. In Algorithm 5, DSW size was set to 150 , and the algorithm samples every $30^{\text {th }}$ frame if $\mathrm{AoC}$ at the beginning of the current DSW is less than $0.015\left(T_{l}\right)$. The algorithm considers all the frames in the current DSW as AoC grows higher than $0.03\left(T_{h}\right)$. The values of $T_{h}$ and $T_{l}$ were estimated by analyzing $\mathrm{AoC}$ in a video recording taken from the scene for a specific period of time (review Section 4.2.1). 


\subsubsection{Evaluation of Execution Time}

In this experiment, the evaluation includes running the prototypes of SimpleCD, $S h C D, S d C D$, and $S h d C D$ on 1, 2, 4, and 8 nodes to investigate the performance as the degree of parallelism increases. The two sets of experiments for both scenarios (weekday and weekend) have been conducted. The video segments were distributed evenly among the cluster nodes. For both scenarios, 32 video segments/files of size $128 \mathrm{MB}$ were used. The experimental results for the weekday scenario are presented in Table B.13. Figure 5.2 shows how the execution time decreases as the number of nodes increases. The $\mathrm{x}$-axis represents the number of nodes, and the $\mathrm{y}$-axis represents the execution time in seconds. The figure reveals that for any given number of nodes, the execution time of ShdCD is lower than that achieved with the other approaches. It also shows that the improvement achieved by sampling the data frames $(S d C D)$ is significantly larger than the improvement achieved by sampling the history frames $(S h C D)$. This is because the number of data frames that benefit from sampling is significantly larger than the number of history frames.

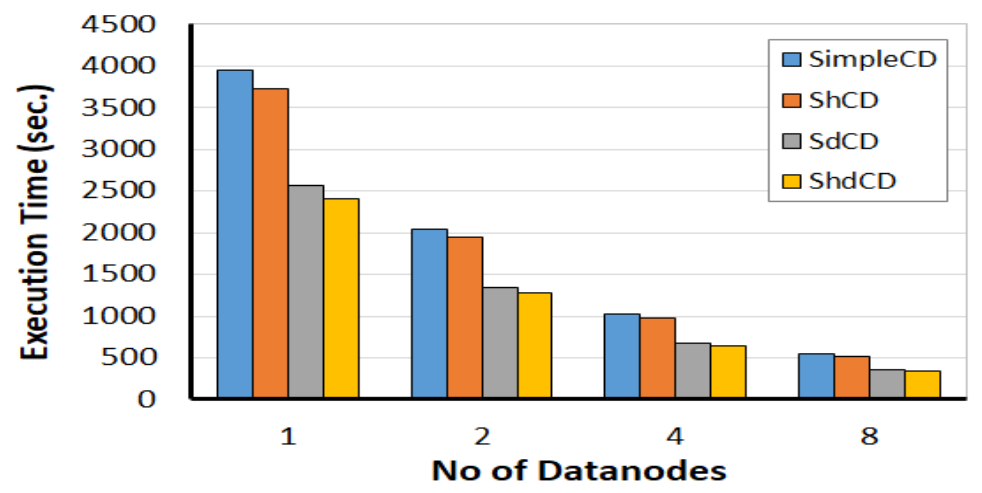

Figure 5.2: Degree of parallelism and execution time (weekday).

Figure 5.3 shows the relationship between the degree of parallelism and the execution time improvement for all algorithms. The y-axis represents the reduced execution time as a percentage in comparison with the execution time of SimpleCD. ShdCD 
shows the best performance because it uses sampling for both history and data frames. For example, for 8 nodes, ShdCD shows a reduction of $37.5 \%$ in execution time that was achieved with SimpleCD.

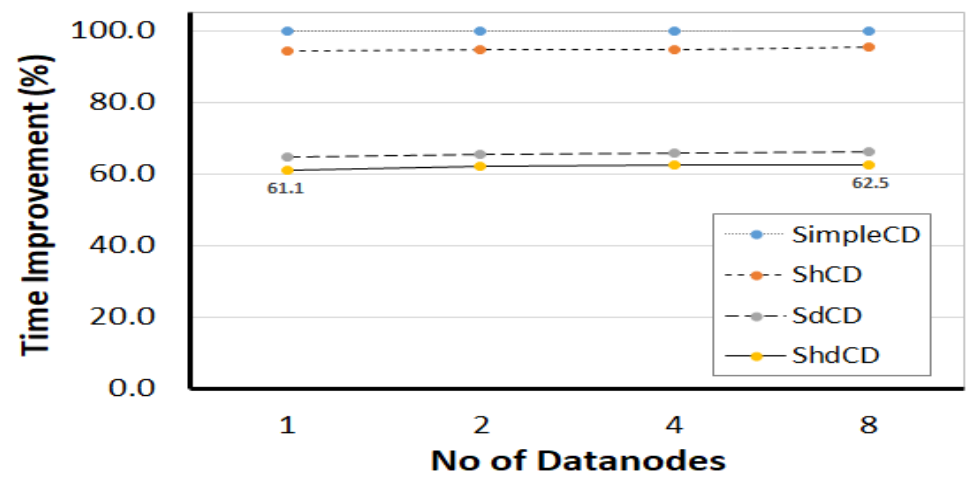

Figure 5.3: Degree of parallelism and execution time improvement (weekday).

As shown in the table, all algorithms achieve almost similar parallel speedup, and Figure 5.4 depicts SimpleCD and ShdCD. Figure 5.5 shows a comparison in terms of efficiency between SimpleCD and ShdCD. Both the algorithms show a comparable efficiency that the efficiency marginally decreases as the degree of parallelism increases.

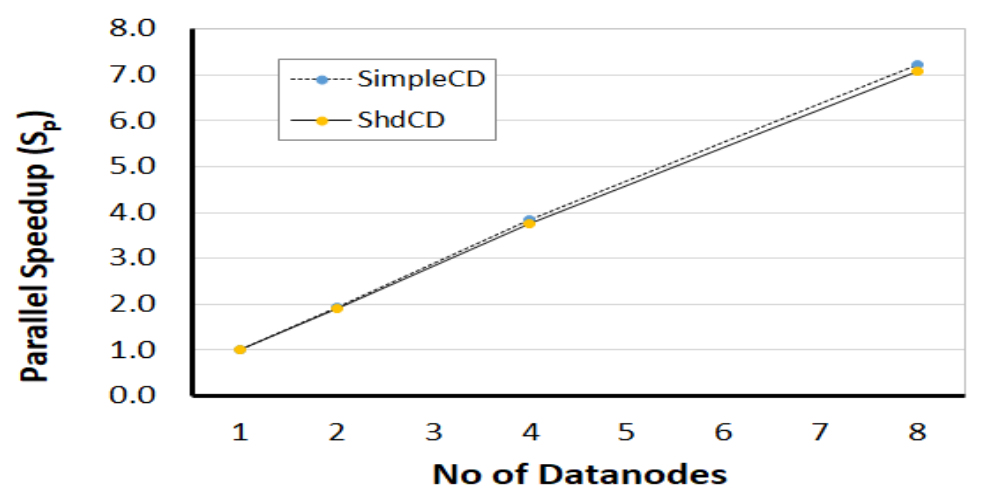

Figure 5.4: Degree of parallelism and speedup (weekday). 


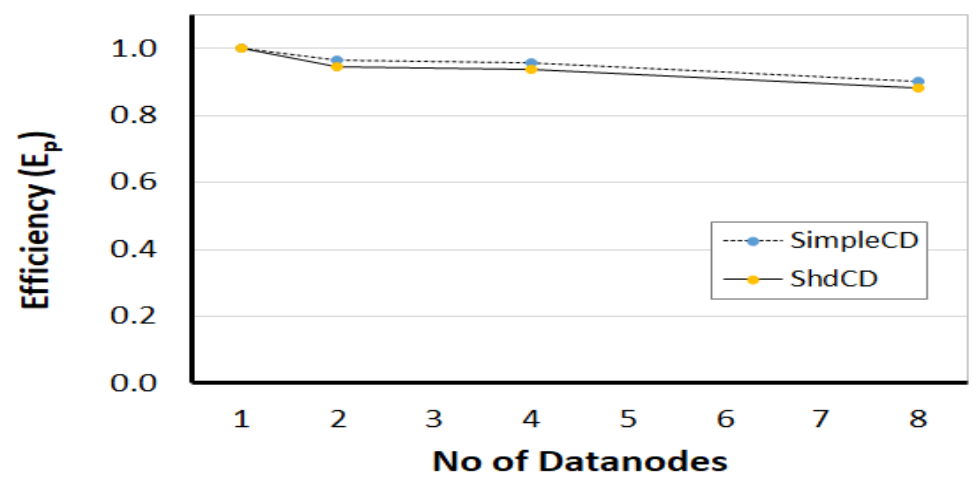

Figure 5.5: Degree of parallelism and efficiency (weekday).

The execution time of SimpleCD decreases from 3947 seconds (on one node) to 546 seconds on 8 nodes achieving a speedup of 7.2 approximately. ShdCD reduces the execution time to 341 seconds on 8 nodes achieving a speedup of 11.6, while $S h C D$ and $S d C D$ achieve a speedup of 7.6 and 10.9 on 8 nodes, respectively. It is important to note that the achieved speedup is a result of effectively performing both parallel computing and sampling.

Table B.14 demonstrates the experimental results for the weekend scenario. Figure 5.6 shows a behavior similar to that of the weekday scenario: with an increase in the degree of parallelism, the execution time decreases, and $S h d C D$ outperforms the other algorithms. The execution time improvement is captured in Figure 5.7, and it shows that $S h d C D$ outperforms the other algorithms irrespective of the number of nodes $(1,2,4$, or 8$)$. 


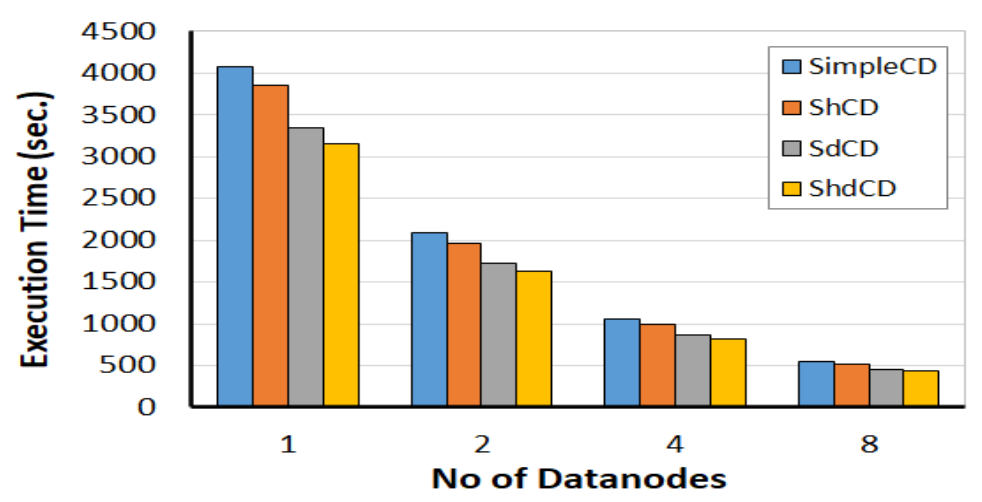

Figure 5.6: Degree of parallelism and execution time (weekend).

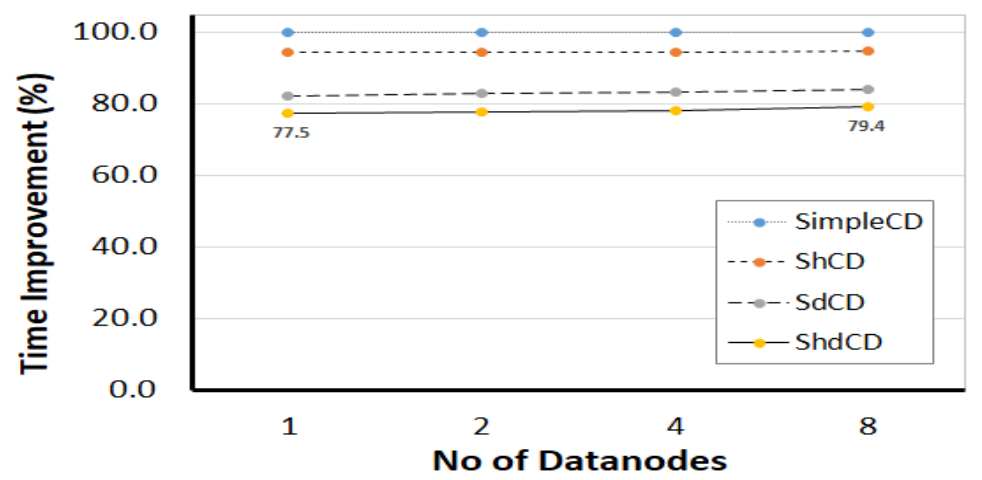

Figure 5.7: Degree of parallelism and execution time improvement (weekend).

The parallel speedup of SimpleCD and ShdCD can be observed in Figure 5.8, while the results for the rest of the algorithms are captured in the Table. Figure 5.9 shows the efficiency achieved for both SimpleCD and ShdCD. On 8 nodes, ShdCD achieves a speedup of up to 9.4 outperforming the other algorithms, SimpleCD, $S h C D$, and $S d C D$, that achieve speedups of up to $7.5,7.9$, and 8.9, respectively. 


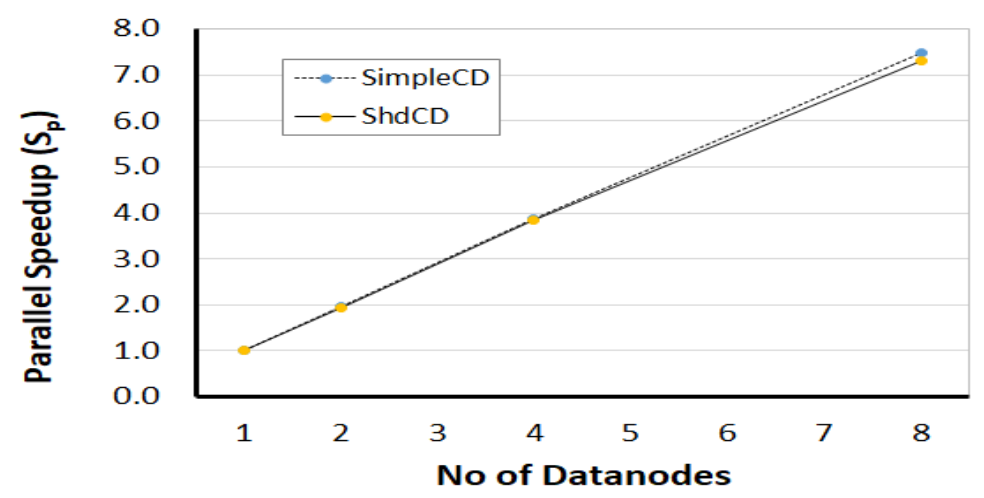

Figure 5.8: Degree of parallelism and speedup (weekend).

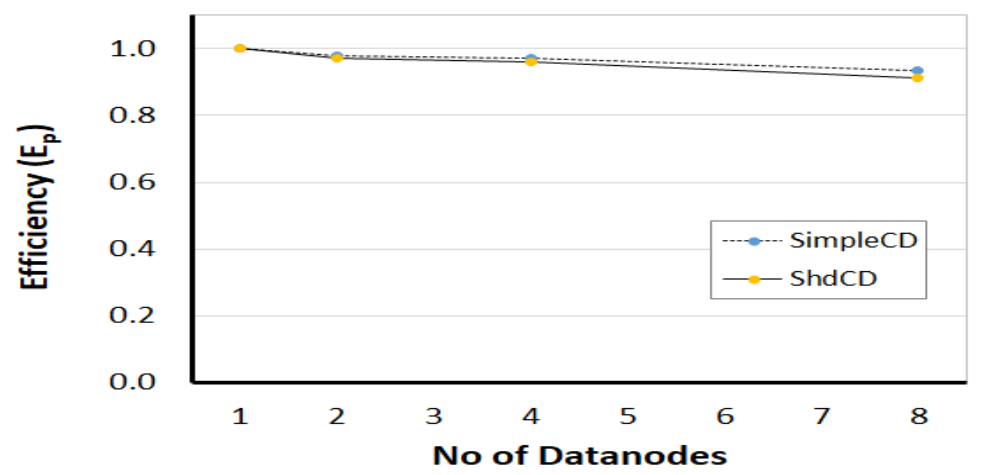

Figure 5.9: Degree of parallelism and efficiency (weekend).

\subsubsection{Impact of the Video Segment Size}

The goal of this experiment is to study the impact of the video segment size (128 MB, $256 \mathrm{MB}$, and $512 \mathrm{MB}$ ) on the performance. The segments were distributed among 8 Hadoop nodes evenly, and the results have been detailed in Table B.15 for the weekday scenario and Table B.17 for the weekend scenario. In the weekday scenario, Figure 5.10 depicts the execution time in seconds (y-axis) as a function of the used approach (x-axis). The results reveal that the execution time slightly improves with the larger segment size. This is because having fewer video segments of large size incur fewer overlapping frames (the source of the overhead). 


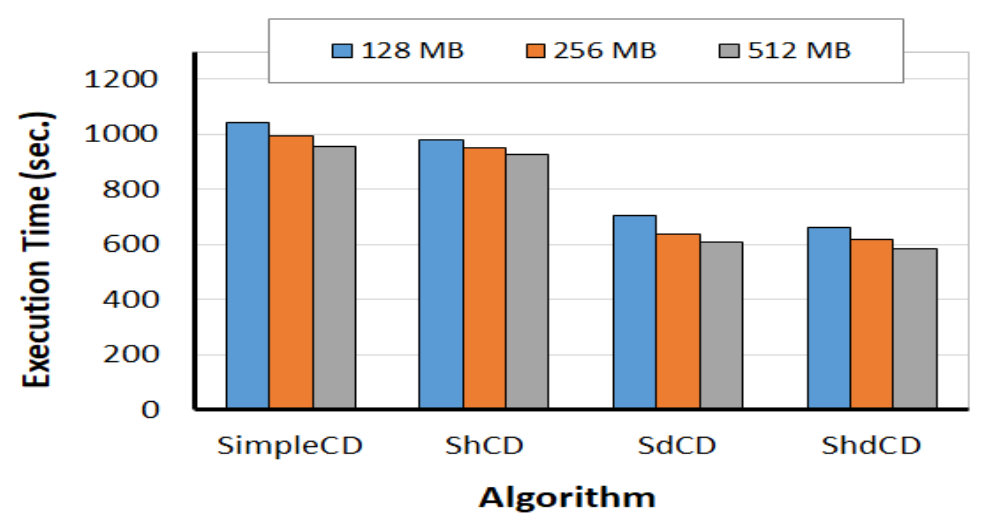

Figure 5.10: Segment size and execution time (weekday).

Figure 5.11 shows the percentage of execution time improvement incurred (y-axis) because of using a large video segment size for all approaches. The figure shows how the improvement achieved by $S h d C D$ outperforms the other approaches; more details are depicted in Table B.16.

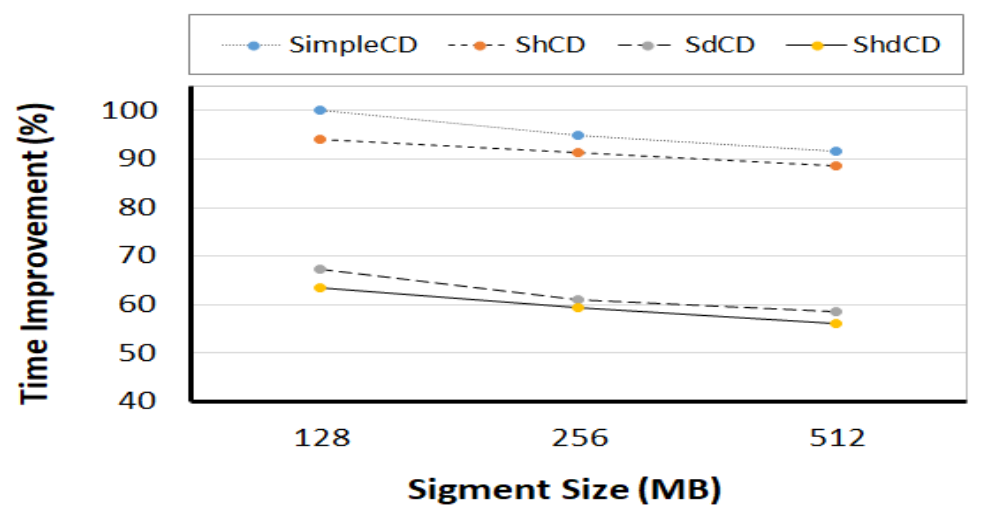

Figure 5.11: Segment size and execution time improvement (weekday).

Figure 5.12 shows the execution time using different video segment sizes for the weekend scenario. As expected, using a larger video segment size reduces the execution time for the reason that fewer overlapping frames (overhead) will be incurred. 


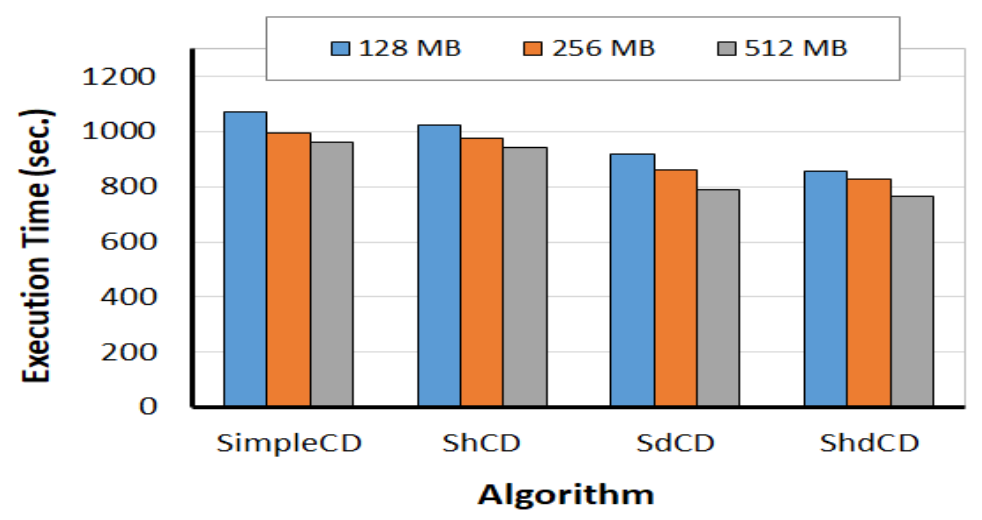

Figure 5.12: Segment size and execution time (weekend).

The execution time improvement is depicted in Figure 5.13, and the figure demonstrates how ShdCD outperforms the rest of the algorithms; more details are depicted in Table B.18.

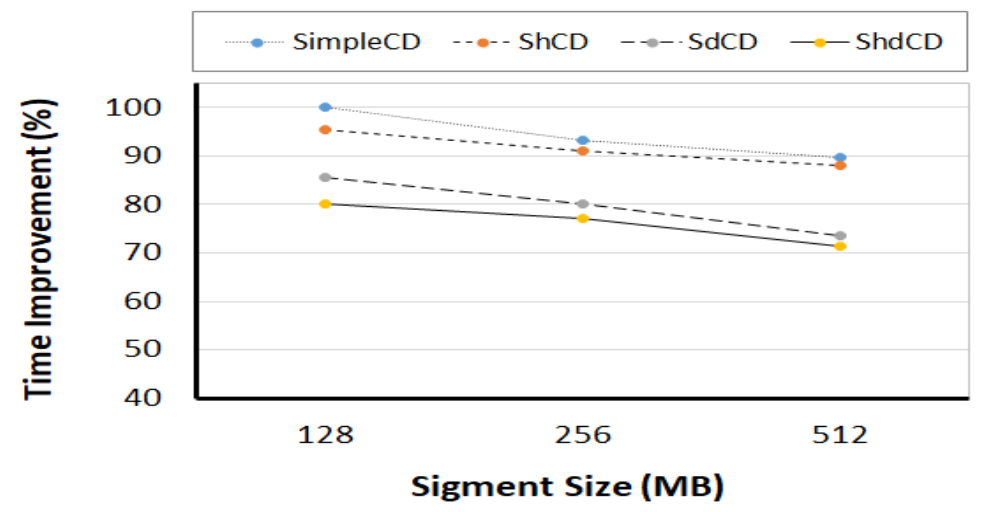

Figure 5.13: Segment size and execution time improvement (weekend).

\subsubsection{Impact of Input Data Size}

This experiment investigates the impact of the input video data size on the execution time of all approaches. Both weekday and weekend scenarios have been considered and experimented on 8 datanodes. The experimental results for the weekday scenario have been collected in Table B.19. Figure 5.14 depicts the relation between the number of processed video files (x-axis) and the incurred execution time in seconds 
(y-axis). The algorithms that depend on sampling data frames show significantly less execution time compared to the other algorithms. The figure also shows that $\operatorname{ShdCD}$ outperforms the other algorithms, whatever the number of processed video files.

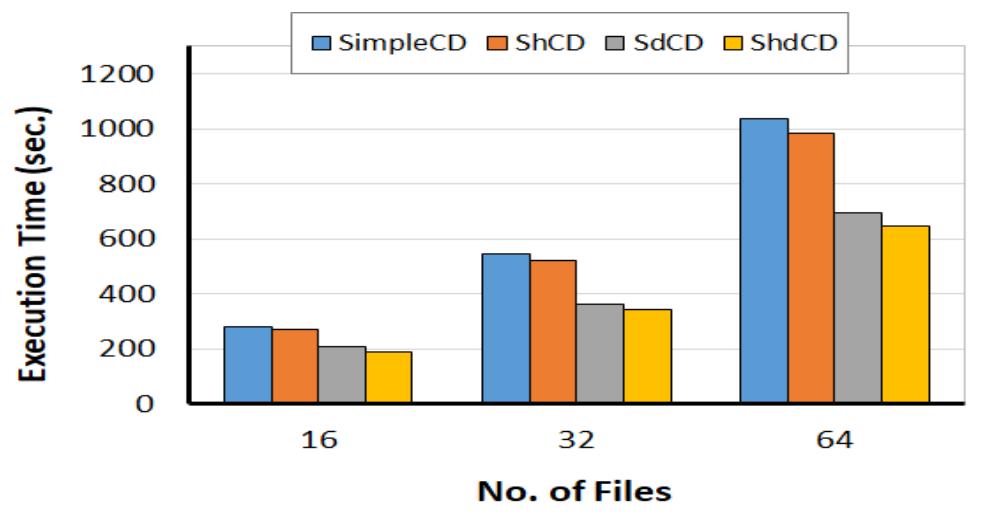

Figure 5.14: No. of video files and execution time (weekday).

For the weekend scenario, the experimental statistics have been illustrated in Table B.20. Figure 5.15 manifests that the execution time decreases when $\operatorname{ShdCD}$ is used in all cases $(16,32$, and 64 files). It also shows that the execution time improvement is less than the improvement achieved with the weekday scenario (see Figure 5.14). This is because, in the weekend scenario, the scene is congested with people more than the weekday scenario; this means that the effect of the sampling technique is higher when the weekday scenario is processed.

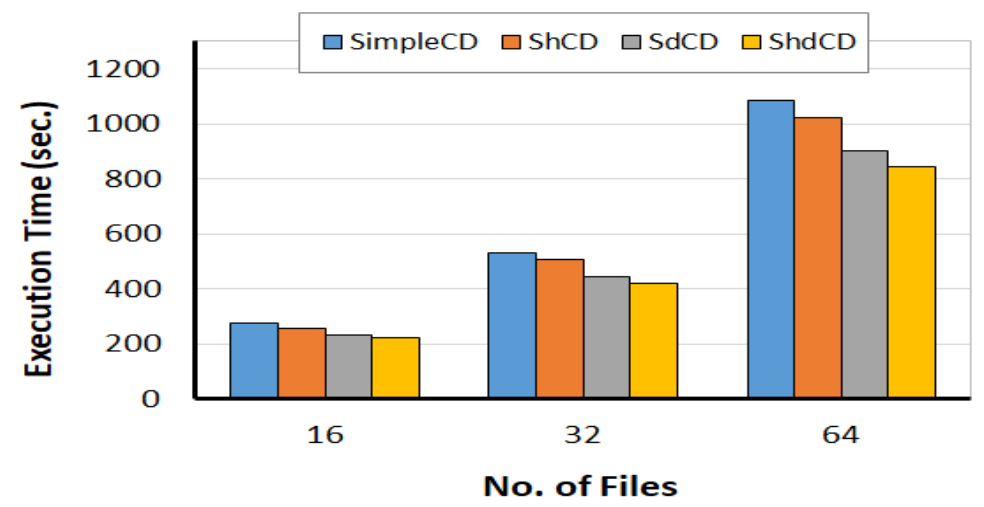

Figure 5.15: No. of video files and execution time (weekend). 


\subsubsection{Evaluation of Fault Tolerance}

This experiment investigates the impact of node failures on the execution time of all approaches. The number of nodes used in this experiment was fixed at 8 . The replicas of all video segments were distributed evenly among the datanodes in such a way that the replicas of the same segment never be located in the same node. In a one-node failure experiment, two replicas for each video segment were used to support fault tolerance. Similar to [78], the node failure experiment was conducted such that after $50 \%$ of the job execution, a randomly selected node was killed to measure the execution time deterioration. Figure 5.16 depicts the execution time in seconds (y-axis) as a function of the different approaches (x-axis). This experiment includes the three video segment sizes (128 MB, $256 \mathrm{MB}$, and $512 \mathrm{MB})$. In the case of no-fault, using the large video segment shows slightly better performance than using the small video segment for all approaches (see Figure 5.10). In the case of a node failure, the results show that the execution time increases in all approaches as the segment size increases (see Table B.21 for more details). The reason is that the more small segments exist on the faulty node, the more working nodes can participate in processing those segments.

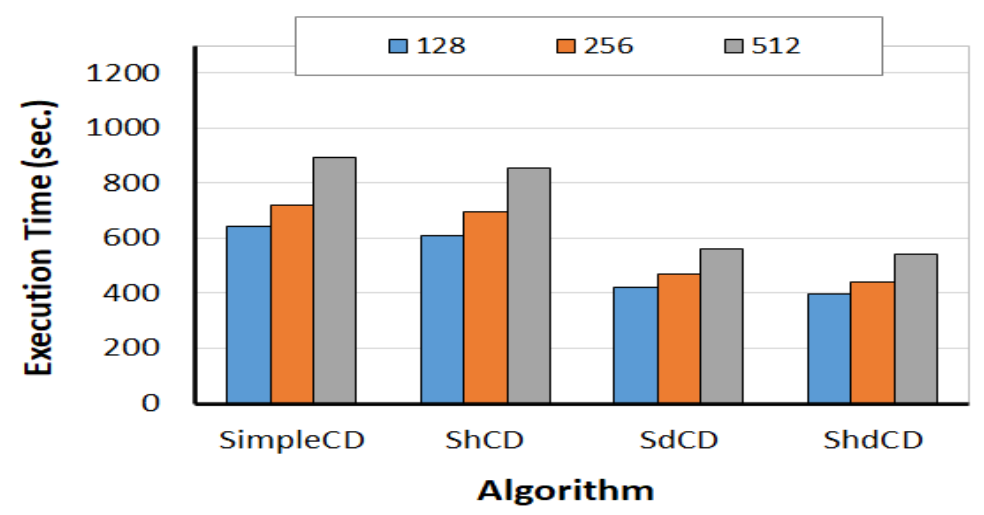

Figure 5.16: One-node failure recovery and execution time.

Figure 5.17 shows the execution time deterioration as the segment size increases. 
In all approaches, using a large segment size is slightly better than using a small segment size in the absence of failure, while using a small segment size is significantly better in case of a node failure. More details are captured in Table B.22.

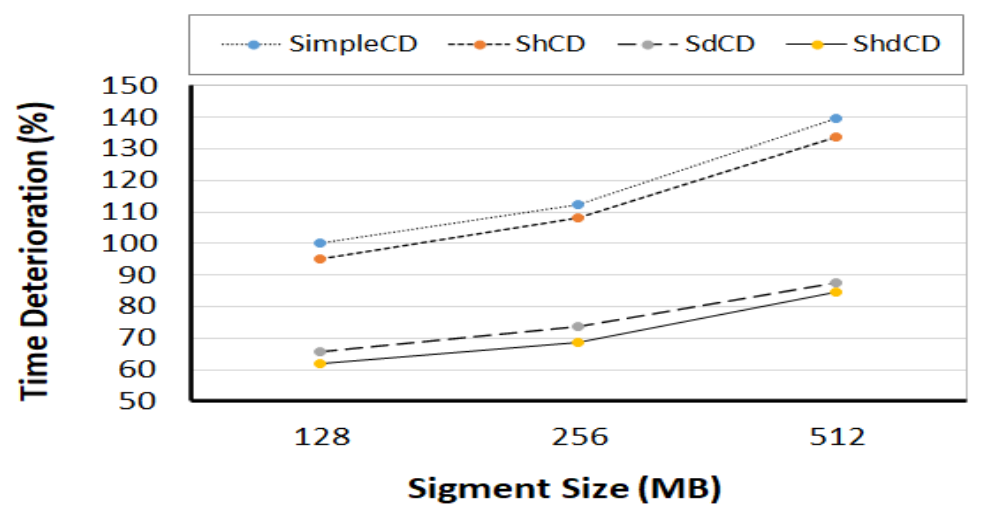

Figure 5.17: One node failure recovery and execution time deterioration.

Figure 5.18 focuses on the effect of Point of Failure (PoF) on execution time. PoF is the percentage of a given job that has completed before at least one node becomes faulty. The figure shows that PoF does not affect the execution time of the job. If the failure occurs after $25 \%, 50 \%$, or $75 \%$ of the execution time, the execution time after recovery remains almost the same. This is because the data blocks in the faulty node will be preprocessed on the working nodes after they complete processing the already designated data blocks. The details are shown in Table B.23.

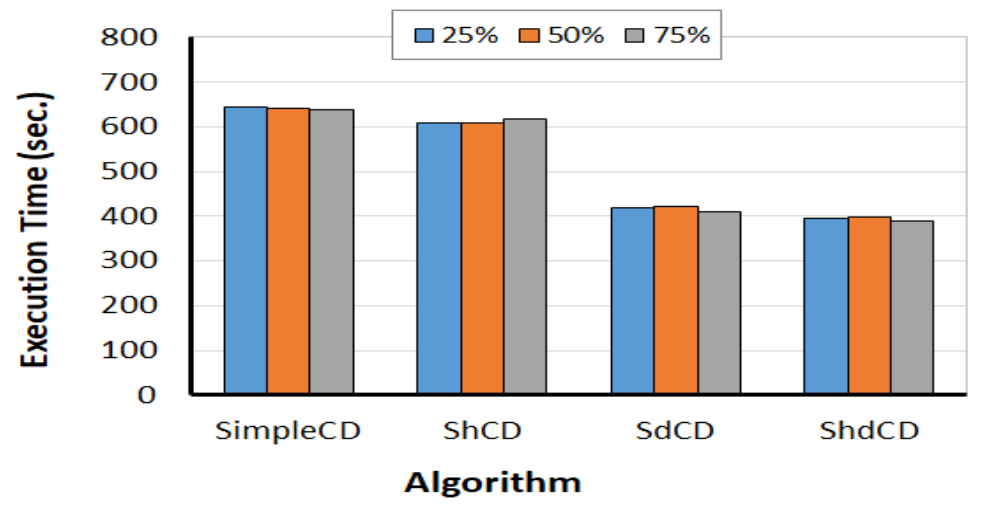

Figure 5.18: One-node failure recovery and point of failure (PoF). 
Figure 5.19 shows the effect of two-node failures on the performance in the weekday and weekend scenarios. In this experiment, failure occurs after the job has completed $50 \%$ of the execution time $(\mathrm{PoF}=50 \%)$. The figure shows that $S h d C d$ outperforms the other algorithms in both scenarios (more details in Table 5.19). It also shows that the execution time is better in the case of the weekday scenario when $\operatorname{ShdCD}$ and $S d C D$ were used. This is because the scene is not crowded with people; hence, more data frames will be sampled.

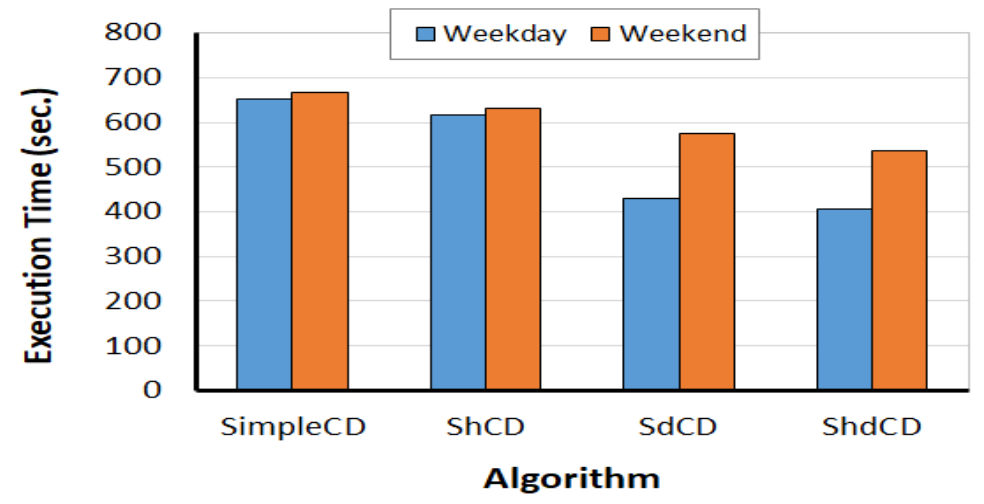

Figure 5.19: Two-node failure recovery and execution time.

\subsection{Chapter Summary}

This thesis discusses how to improve the performance of MapReduce-based change detection by using different sampling techniques so that large video data can be processed in a timely manner. One sampling technique is to minimize the extra computation resulting from the overlapping frames of each video segment. The second sampling technique is to reduce the computation time for CD by considering some data frames and ignoring the others based on the amount of changes in the scene. The technological innovation introduced in this research involves three algorithms that use the proposed sampling techniques. The performance was studied empirically by running proof of concept prototypes for the three algorithms on a Hadoop 
cluster on Cloud resources. The experimental results show that ShdCD improves the performance of MapReduce-based CD by saving up to $35 \%$ of the execution time in comparison to SimpleCD on 16 Hadoop nodes. Furthermore, ShdCD outperforms SimpleCD in the fault-tolerant scenario discussed in this chapter. 


\section{Chapter 6}

\section{Data Placement Policy}

HDFS stores and replicates data blocks according to the default Data Placement Policy (Default-DPP) such that no two replicas of the same block coexist in the same datanode, and no more than two replicas for the same block exist in the same rack, in case there is more than one rack [13]. Since the default data placement policy does not take into account the disk space utilization of datanodes, it results in storage load imbalance. This problem can be handled by using a tool known as Balancer [13] to balance the storage load.

Processing video data in a Hadoop cluster requires partitioning the data into segments/files of size smaller than HDFS block size before uploading the files to HDFS. In video processing, it is common that applications process only the Region of Interest, so processing two equal-sized video segments most likely requires different workloads. Therefore, the MapReduce implementation of such processing inevitably causes workload imbalance when the default data placement policy of Hadoop is used to distribute video data. The change detection algorithm can be modified such that it avoids unnecessary computation, as described in Chapter 4. Even if the video data are distributed evenly across cluster nodes, and the cluster is homogeneous, running the modified change detection results in workload imbalance as well as access load imbalance. The workload imbalance is when two datanodes, for example, process the 
same size of video data, but they require different execution times since the workloads depend on the content of the video data. The access load imbalance occurs when some datanodes process the data locally, while others need to transfer remote data to be processed locally. The desired data placement policy should minimize data transfer on the network (i.e., minimize access load imbalance), especially on clouds. This chapter focuses on devising three data placement policies to minimize the access load imbalance resulting from change detection based applications in homogeneous Hadoop clusters.

\subsection{Modified Change Detection Approach}

Change Detection can be modified such that not all video frames are considered in updating the background model. The reason for this is to avoid unnecessary computations in case there are no significant changes in the scene, hence reducing the execution time. This approach uses MapReduce to distribute processing and a sampling strategy to avoid unnecessary computations. This approach is termed a Nonuniform MapReduce-based CD (NU-MR). Both SdCD and ShdCD approaches (see Chapter 5) can be used to implement NU-MR since they sample the data frames. In this chapter, ShdCD will be used to implement NU-MR.

The sampling strategy is devised to decide whether to update the background model with every frame in a video segment or to use sampling, based on $\mathrm{AoC}$ in the scene. AoC represents the number of the white pixels divided by the total number of the pixels in the computed binary mask frame $f_{b m}^{t}$. To achieve the sampling strategy, a double-threshold algorithm is devised as follows. First, for a given video segment $\left(V^{j}\right)$, all the history frames $\left(V_{h}^{j}\right)$ are used to estimate the initial background model

$\left(f_{b g}^{0}\right)$. Second, the data frames $\left(V_{d}^{j}\right)$ of the segment are logically divided into windows of size $w$. Third, at the beginning of each window, AoC in the current data frame 
$f_{d}^{t}$ is estimated. Forth, the algorithm uses every frame in the current window to update the background model if the estimated AoC is larger than a predefined high threshold $\left(T_{h}\right)$. On the other hand, if AoC is smaller than a predefined low threshold $\left(T_{l}\right)$, the algorithm will consider every $i^{\text {th }}$ frame in the current window to update the background model. Finally, the third and fourth steps will be iterated for every window in the video segment.

\subsection{Proposed Data Placement Policies}

\subsubsection{Challenge}

Using the default data placement policy to distribute a set of video files is not effective in case these files are required to be processed together. This is because the files will be stored randomly in datanodes causing the problem of access load imbalance. This thesis refers to such a set as a related dataset or related video segments/files.

The problem of access load imbalance can be solved by increasing the replication factor; however, the larger the replication factor, the more resources will be wasted. Solving the problem by evenly distributing data blocks may not reduce the data transfer overhead when a related dataset is processed using ShdCD jobs. To demonstrate this, assume that there is a related dataset recorded for people moving in a public area for a period of time. Also, assume that the related dataset includes 16 video segments/files, and the workloads required for them are not the same, as shown in Figure 6.1(a). This means that the scene is not crowded at the beginning (low $\overline{A o C}$ ), and then it becomes crowded over time (high $\overline{A o C}$ ). $\overline{A o C}$ is the average value of all data frames' AoC of a video segment, and it signifies whether the segment requires a high workload (high value) or low workload (low value). An important observation to be made here is that $\overline{A o C}$ in consecutive video segments varies gradually in time. 
For instance, in the skating scenario, the video recording collected on the weekend shows that there were a few skaters in the morning. As the time approached the noon period, the number of skaters increased gradually. Li et al. [76] observed similar situations in a traffic scenario, and they concluded that video data collected from the same location and the same period usually cost similar processing times. Such a gradual change in the scene is expected for various videos, including surveillance videos. Figure 6.1(b) shows a naive approach to distribute the data blocks of the 16 related video files to 4 datanodes. The blocks of the 16 video files are evenly distributed to the 4 datanodes such that the first 4 data blocks are stored in datanode 0 , the second 4 data blocks are stored in datanode 1, and so on. This approach is termed as Naive-Even-Distribution Data Placement Policy(NED-DPP). As shown in Figure 6.1(c), NED-DPP results in data migration when data blocks $B_{11}$ and $B_{15}$ transfer to datanode 0 and 1 , respectively. In sum, it is noticeable that NED-DPP still results in both workload imbalance and access load imbalance.

\subsubsection{Content-wise Data Placement Policy}

There are three aspects that need to be considered in distributing the video data that will be processed using ShdCD approach as follows. First, the data blocks of a related dataset will be usually accessed together; therefore, they need to be uniformly distributed. Second, the data blocks may require different workloads. Third, $\overline{A o C}$ in consecutive video segments varies gradually in time (see Figure 6.1(a)).

The proposed data placement policy, called Content-wise Data Placement Policy (CW-DPP), has been designed to support ShdCD approach by minimizing the data transfer overhead and balancing the workload, as shown in Figure 6.1(d). It is based on the round-robin strategy that can satisfy the previously mentioned aspects. The advantage of using round-robin is that it balances the workload by distributing both data blocks with high $\overline{A o C}$ and data blocks with low $\overline{A o C}$ evenly. 


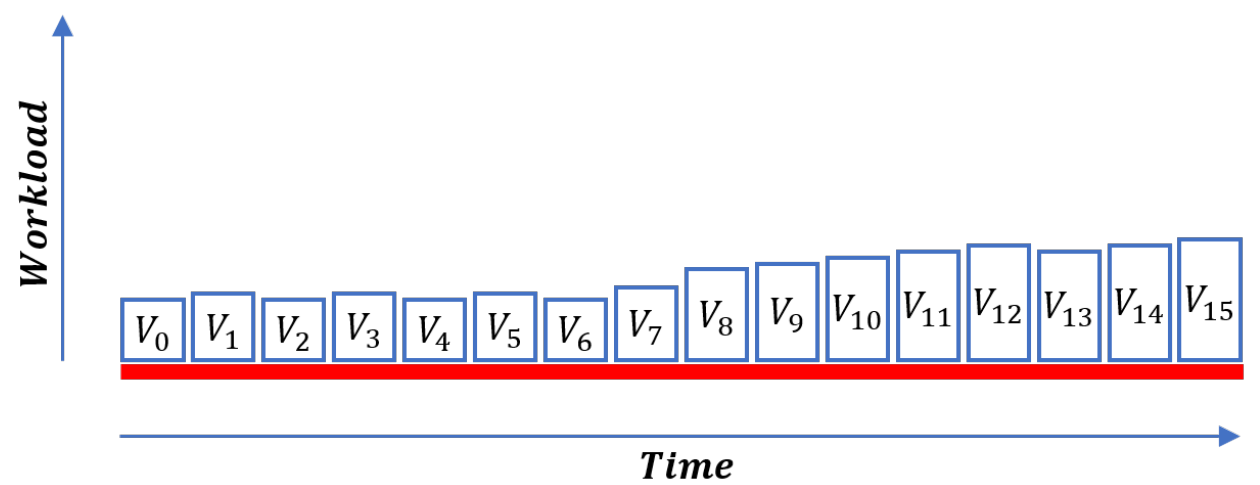

(a)

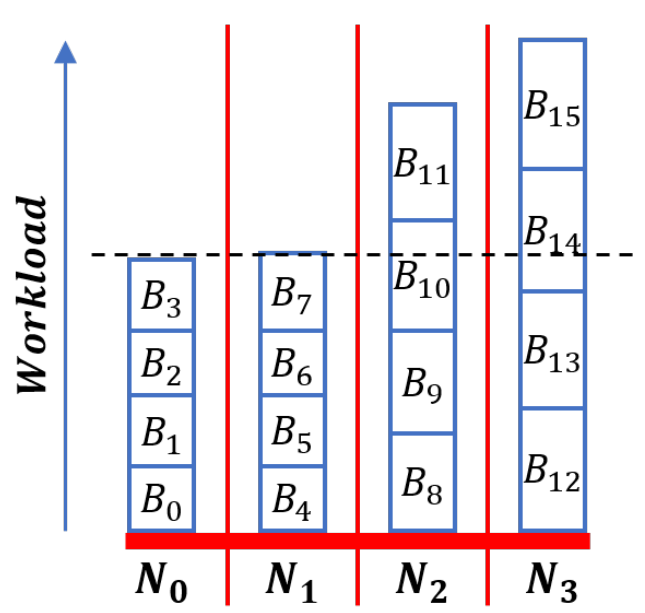

(b)

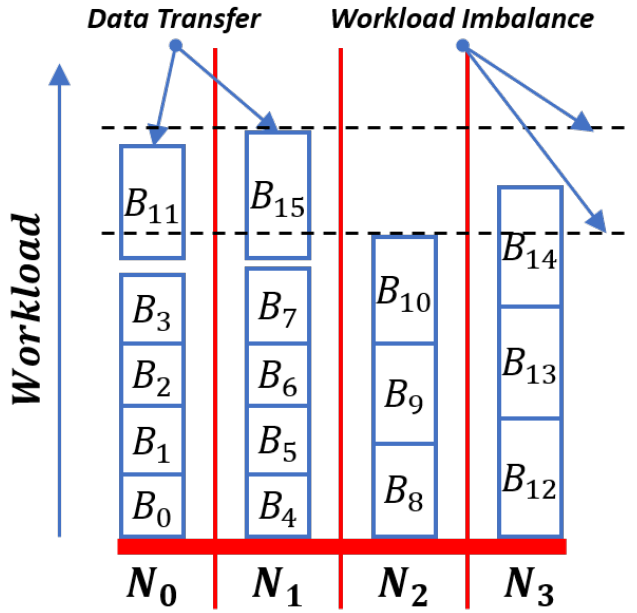

(c)

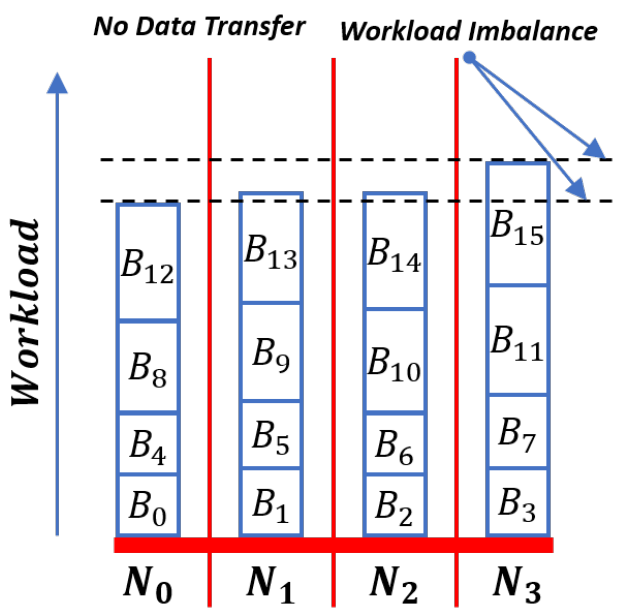

(d)

Figure 6.1: Example of Data blocks placement. 
The idea of CW-DPP is basically to divide $n$ datanodes of a Hadoop cluster into two groups $G_{0}$ and $G_{1}$, where $G_{0}$ includes one half of the datanodes $\left\{N_{0}, N_{1}, \ldots, N_{n / 2-1}\right\}$, and $G_{1}$ includes the other half $\left\{N_{n / 2}, N_{n / 2+1}, \ldots, N_{n-1}\right\}$. For modeling simplicity, the number of datanodes is assumed to be a multiple of 2 ; therefore, the size of each group is $g=n / 2$. To store each video segment of a related dataset, the first data block (replica) has to be stored in a group while the second replica should be stored in the other group. If the replication factor is one, the algorithm distributes the data blocks to the datanodes of the two groups in a round-robin fashion. Although using one replica reduces the cost on Cloud platforms, it will not support recovery from a node failure.

To achieve the previous idea, there is a data structure for each group, called a utilization list, used to hold information about the datanodes belonging to that group. Let $U_{0}$ and $U_{1}$ denote the utilization lists for $G_{0}$ and $G_{1}$, respectively, and the size of each list equals to the size of its group, $g$. The information is stored as $g$-tuple of $\langle$ index $|$ load $\rangle$ values such that each tuple represents a datanode. The index value refers to a specific datanode, and the load value is the number of the data blocks stored in the datanode. The information of datanodes is sorted in non-decreasing order based on the load values.

Algorithm 11 describes the content-wise data placement policy. The datanodes of each utilization list are sorted in non-decreasing order based on the number of the stored replicas on each datanode (Line 2). The algorithm selects the group to start placing the video segments in its datanodes by comparing the first element of the two utilization lists $\left(U_{0}\right.$ and $\left.U_{1}\right)$. For example, if the 'load' value of the first element in $U_{0}$ is smaller than the first element in $U_{1}, G_{0}$ will be selected, otherwise $G_{1}$ (Line 3). If the replication factor is ' 1 ', only the first replicas of the video segments will be distributed among the datanodes in a round-robin fashion (Line 4). However, if the replication factor is ' 2 ' (Line 5), the first replicas of the first $g$ video segments 


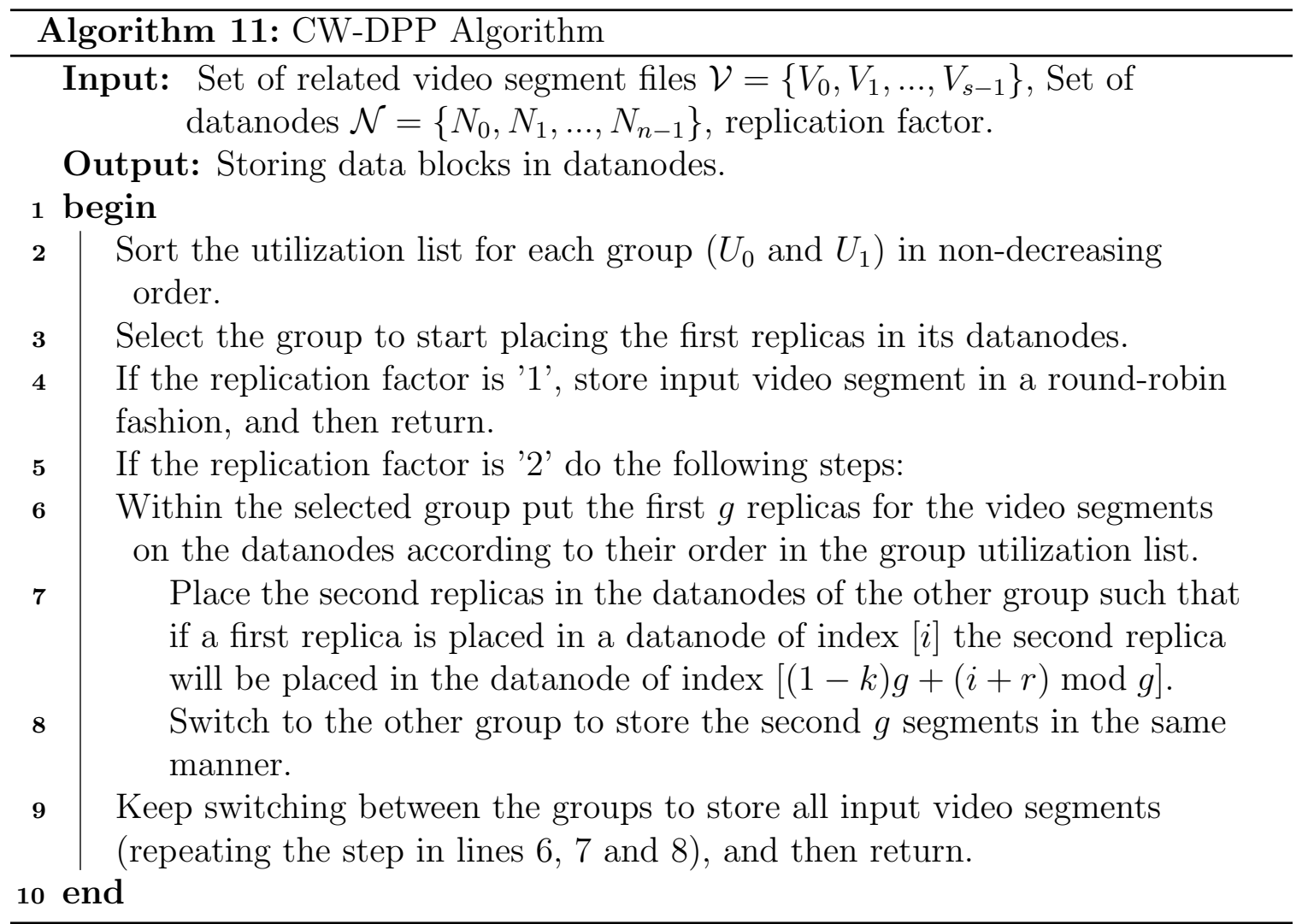

will be stored among the datanodes of the group selected in Line 3 according to the utilization list of the group (Line 6). If the first replica of a video segment is stored in a datanode of index $[i]$, the second replica is to be stored in the datanode of index $[(1-k) g+(i+r) \bmod g]$, where $k$ is the current group and $r$ is the current round (Line 7). The algorithm (in Line 8) switches to the other group to store the first replicas of the second $g$ video segments according to the group utilization list, and store the second replicas of the same video segments according to the index determined in Line 7. Finally, the algorithm will keep switching by repeating the steps in Lines 6,7 and 8 until storing the replicas for all video segments (Line 9). Further details are included in Appendix A.4.

Figure 6.2 is an example to demonstrate how CW-DPP stores data blocks in a Hadoop cluster consisting of 8 datanodes. The first 4 datanodes form $G_{0}$, and 
the rest of the datanodes form $G_{1}$. Initially, the contents of the utilization lists are: $U_{0}=(\langle 0 \mid 0\rangle,\langle 1 \mid 0\rangle,\langle 2 \mid 0\rangle,\langle 3 \mid 0\rangle)$, and $U_{1}=(\langle 4 \mid 0\rangle,\langle 5 \mid 0\rangle,\langle 6 \mid 0\rangle,\langle 7 \mid 0\rangle)$. Assume a related dataset including 16 video segments/files $\left\{V_{0}, V_{1}, \ldots, V_{15}\right\}$ will be stored in the datanodes as data blocks/replicas, where for each video segment $V_{j}$ there are two replicas: $b_{j}^{0}$ and $b_{j}^{1}$. The algorithm selects $G_{0}$ to start assigning the replicas to its datanodes. This is because all datanodes at this point hold no data blocks (Lines 9-13 of Algorithm 17). The first replicas of the first 4 video segments will be stored in the datanodes of $G_{0}$ according to $U_{0}$ (Lines 16-18) while the second replicas will be stored in $G_{1}$ such that if the first replica of a video segment is stored in datanode [i], the second replica will be stored in datanode $[(1-k) g+(i+r) \bmod g]$ (Lines 19-23). Next, the algorithm switches to $G_{1}$ to store the second 4 video segments in the same manner, and it keeps switching until storing all the 16 segments. With every switch, it increases the round counter $r$. The goal of storing 2 replicas in this way is to guarantee that if a datanode fails (e.g., $N_{1}$ in the figure), its replicas are evenly distributed in the other group (e.g., $\left\{b_{1}^{1}, b_{4}^{0}, b_{9}^{1}, b_{14}^{0}\right\}$ in $G_{1}$ ). This mechanism minimizes the data transfer overhead in case of fault tolerance, as will be discussed in Subsection 6.3.2.

\subsubsection{Extended Content-wise Data Placement Policy}

CW-DPP (described in Section 6.2.2) considers using one replica to minimize the cost of the required resources, or two replicas to support one-node failure recovery. In some cases where the stored data are critical, more replicas are required to support the recovery in case there is more than one faulty node. For this reason, CW-DPP has been extended to allow the recovery in case there are two node failures (ECWDPP). To do this, assume that the number of datanodes is a multiple of three, so they can form three equal groups. The previous assumption is reasonable since, on cloud platforms, it is feasible to acquire the required resources to ensure distributing the 


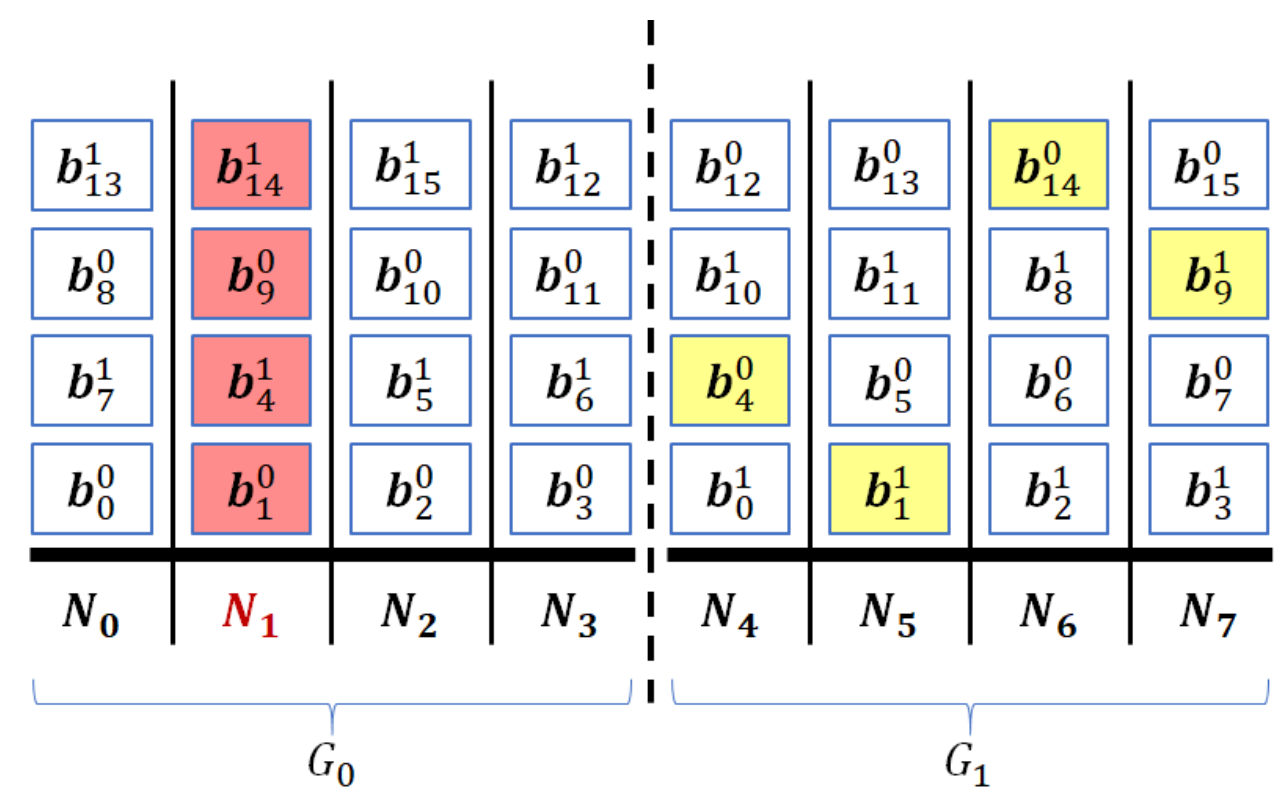

Figure 6.2: An example to demonstrate how CW-DPP works.

data equally among the nodes. For example, we can establish a cluster that includes 300 datanodes (a multiple of 3) so that each group includes 100 datanodes. This means that the computation power of each group is equal. According to this policy, there are three data blocks for each video segment, and they will be distributed among the three groups. Similar to CW-DPP (discussed in Section 6.2.2), the blocks of a related dataset will be stored such that if one node is down, its replicas are distributed evenly among the nodes of the other two groups, hence reducing the data migration overhead in the case of failure. There are three replicas for each video segment, and the three replicas for all segments will be stored as follows. In the first group, $G_{0}$, the first replicas are stored according to a utilization list $U$ that holds a tuple for each datanode belonging to the group. $U$ holds tuples of $\langle$ index $|$ load $\rangle$ values where each tuple represents a datanode. The index value denotes the datanode, and the load value denotes the number of the replicas stored in the datanode. ECW-DPP is presented in Algorithm 12. The tuples in $U$ are stored in non-decreasing order of load 


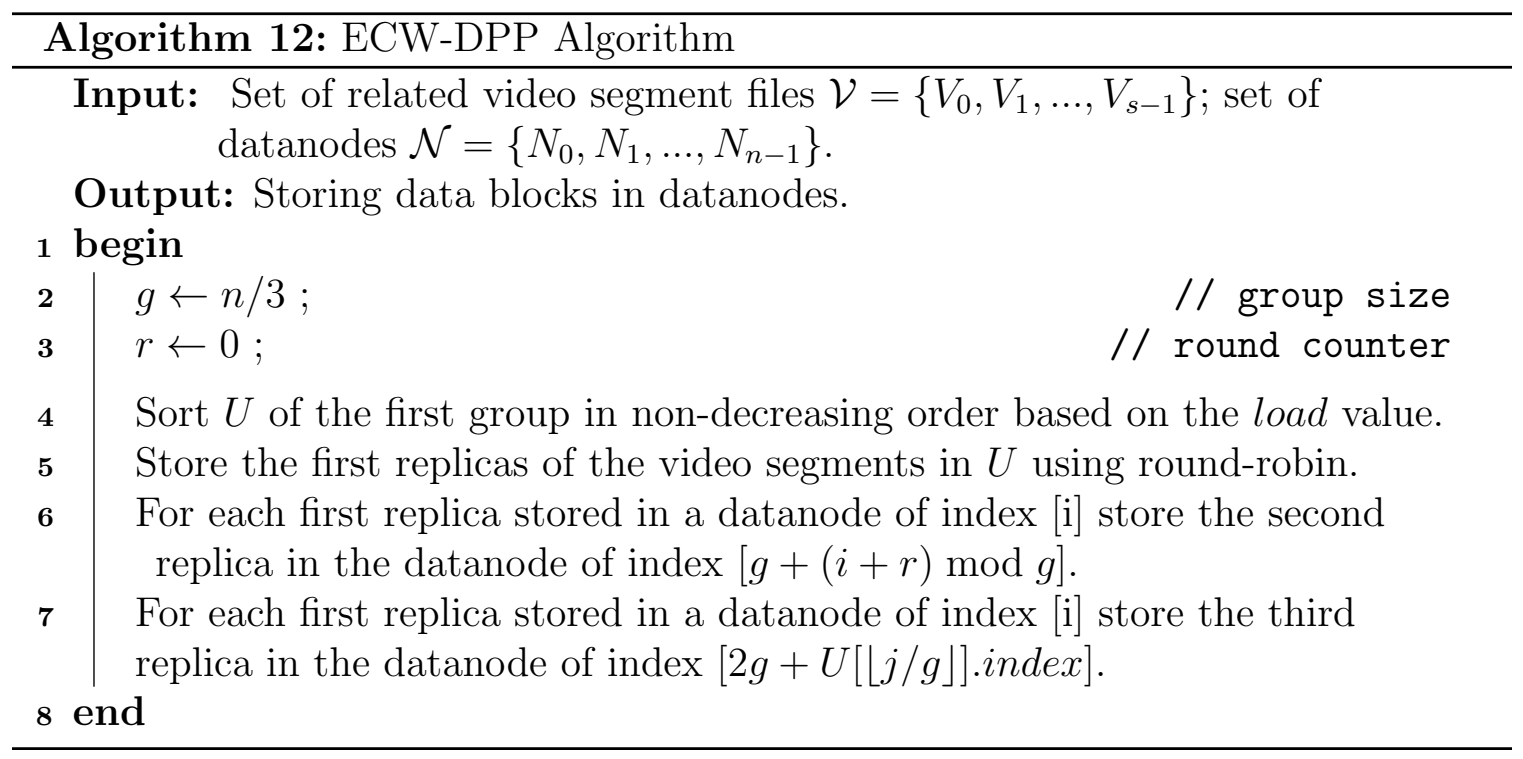

values (Line 4). The first replicas for all video segments will be stored in a roundrobin fashion in $G_{0}$ (Line 5$)$. The second replicas are stored in the second group $\left(G_{1}\right)$ such that if the first replica is stored in a datanode of index $[i]$, the second replica will be stored in a datanode of index $[g+(i+r) \bmod g]$ (Line 6), where $g$ represents the group size, and $r$ represents the round number. The $r$ value starts with '0', and it increases by one after distributing every $g$ video segments. The third replicas will be stored in the third group $\left(G_{2}\right)$ such that if the first replica is stored in a datanode of index $[i]$, the third replica will be stored in a datanode of index $[2 g+U[\lfloor j / g\rfloor]$.index $]$ (Line 7).

\subsubsection{Forward \& Backward Data Placement Policy}

In this section, another algorithm is proposed to reduce the data migration overhead as well as the workload imbalance. The proposed algorithm is an offline algorithm, called Forward \& Backward Data Placement Policy (F\&B-DPP) (see Algorithm 13). According to the algorithm, the execution time for processing each video segment is required to be known a priori. To acquire this information, firstly, we process the 
video segments using the change detection algorithm. Secondly, the execution time of all video segments is used to distribute the video segments across the cluster. This preprocessing step gives rise to an overhead; however, by incurring the overhead once, one can improve the performance of many ShdCD jobs that process the same video segments. The related video segments will be distributed among the datanodes so that we can balance the execution time of all datanodes. Balancing the execution time reduces the data migration overhead and the workload imbalance. Initially, the video segments of a related dataset will be sorted in non-increasing order based on the execution time required to process each segment using ShdCD (Line 2). A utilization list $U$, similar to the list described in Section 6.2.3, is used to store information about the number of replicas stored in each datanode in the cluster. The goal of using $U$ is to minimize the storage load imbalance by assigning the replicas to the data nodes that store less data block first. This goal can be achieved by sorting $U$ in nondecreasing order of storage utilization (load values) (Line 3). The algorithm starts assigning the video segments with high execution time to the datanodes starting from the beginning of $U$ to the end (forward direction, Line 4-5). This means the data block (replica) of the video segment with the largest execution time will be stored in the datanode with the smallest number of stored blocks. The second largest video segment will be given to the second datanode in $U$, and so on. Let $n$ denote the size of the cluster; in other words, there are $n$ datanodes. The algorithm works in rounds, where in the first round, it distributes the first $n$ segments to the datanodes in $U$ in the forward direction (i.e., from the beginning of $U$ to the end). The second $n$ video segments will be distributed in the backward direction (i.e., from the end of $U$ to the beginning). The third round distributes the segments in the forward direction and so on until distributing all video segments (Line 6). If the replication factor is more than one, the replicas of the same video segment will be stored in the consecutive datanodes. For example, in case there are two replicas for the video segment with 


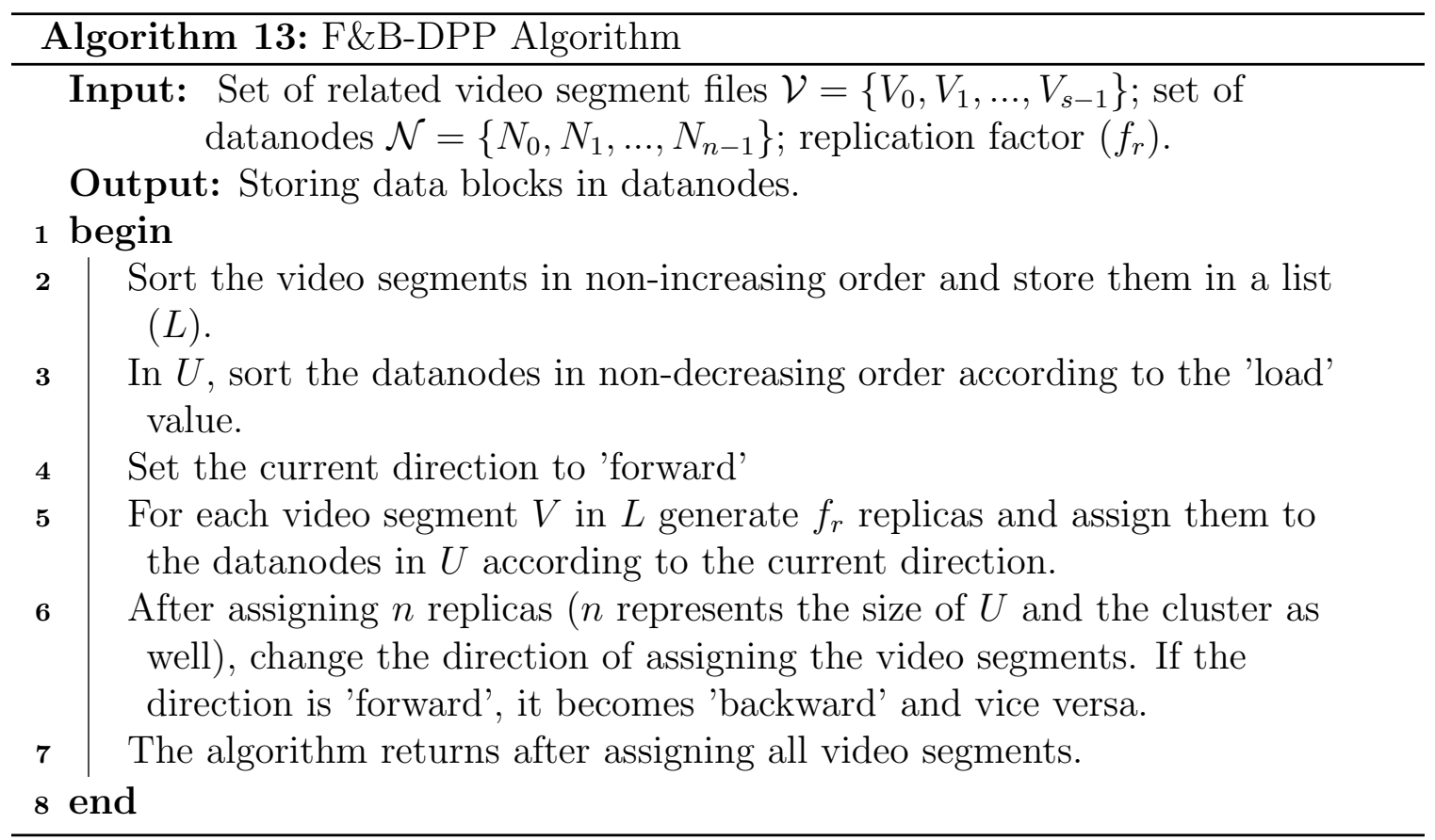

the largest execution time, the first replica will be stored in the first datanode in $U$ while the second one is to be stored in the second datanode.

\subsection{Experiments and Evaluation}

\subsubsection{Experimental Setup and Dataset}

This section describes an empirical study to demonstrate the performance improvement of using ShdCD approach to reduce the execution time of CD and using CWDPP to minimize the data migration overhead. A prototype implementation of ShdCD approach is compared with another prototype implementation of the NormalMR approach in terms of execution time to demonstrate the improvement achieved by ShdCD. The two prototypes are based on map-only jobs that significantly improve the performance [78]. The data locality concept has been used as a metric to evaluate the performance of CW-DPP. The data locality is defined here as the percentage of 
the data blocks processed locally without being transferred; this information can be calculated from the statistics that Hadoop generates for the jobs it runs. High data locality signifies low data migration overhead and hence better performance. The evaluation also includes Default-DPP and NED-DPP for comparison purposes.

6.3.1.1 Dataset The experiments in this study were conducted on video recordings of people skating on a frozen canal. The total size of the recordings is about $12 \mathrm{~GB}$, and the resolution is $720 \mathrm{p}$ with $30 \mathrm{fps}$. The recording is considered a related dataset that was divided into two different video segment sizes: $128 \mathrm{MB}$ and $256 \mathrm{MB}$. There are two scenarios used in this study: weekday and weekend. The weekday scenario was recorded only during weekdays, but the weekend scenario was recorded during both weekends and weekdays. In the weekday scenario, all video segments are not crowded with people, while in the weekend scenario, $50 \%$ of the video segments are crowded with people. The point of this is to study how the proposed algorithms work in different scenarios.

6.3.1.2 Cluster Setup and Technologies The experiments were conducted on Cloud Computing, where a 13-node Hadoop cluster using Amazon EC2 instances was set up. Each instance (t3.xlarge) consisted of 4 vCPUs up to $2.5 \mathrm{GHz}$ Intel Scalable Processor (64-bit), and 16 GB RAM. The network bandwidth was up to 5Gbps. Each instance ran Ubuntu Linux OS (version 18.04 LTS (HVM), 64-bit (x86)), Java VM (OpenJDK version 10.0.2), and Hadoop daemons (Hadoop 2.8.5). One instance was configured as a master (namenode) while the others 12 were configured as workers (datanodes). All nodes were also configured to run OpenCV-based applications. The experiments were repeated three times, at least, and the measurements were within $\pm 5 \%$ of the average. 


\subsubsection{Experimental Results}

Figure 6.3 compares the impact of the four data placement policies on $S h d C D$ approach as the number of processed video files increases in the weekday scenario. The $\mathrm{x}$-axis represents the number of processed video files, and the $\mathrm{y}$-axis represents the job execution time in seconds. The figure demonstrates how CW-DPP and F\&B-DPP reduce the execution time of ShdCD in comparison with the other two data placement policies (Default-DPP and NED-DPP) as the number of input files increases. This is because it improves workload balance.

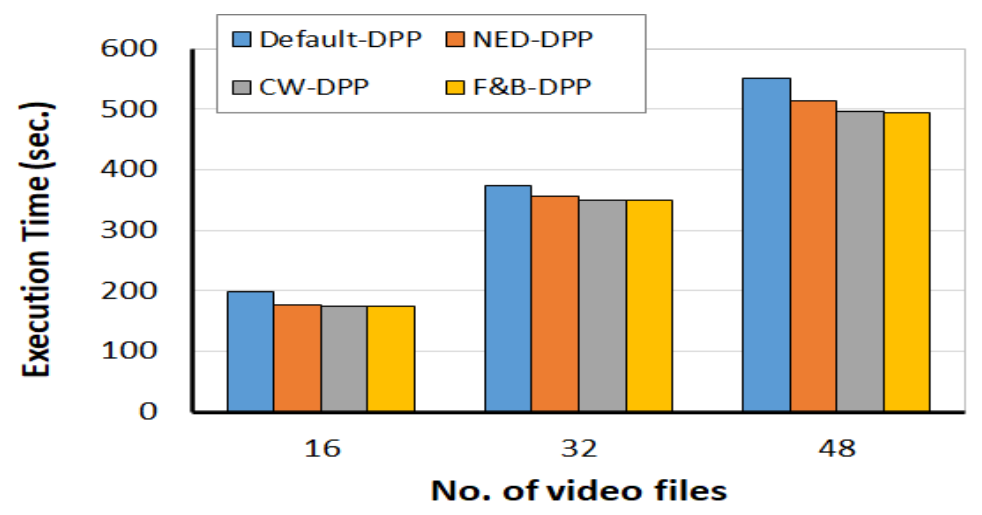

Figure 6.3: Increasing data size and execution time (weekday).

Figure 6.4 shows that when Default-DPP is used, the locality of ShdCD improves as the number of input video files increases; however, the rest of the policies show stable performance. The reason for this is that, in the weekday scenario, the amount of changes are almost similar. Table B.25 demonstrates more experimental results. 


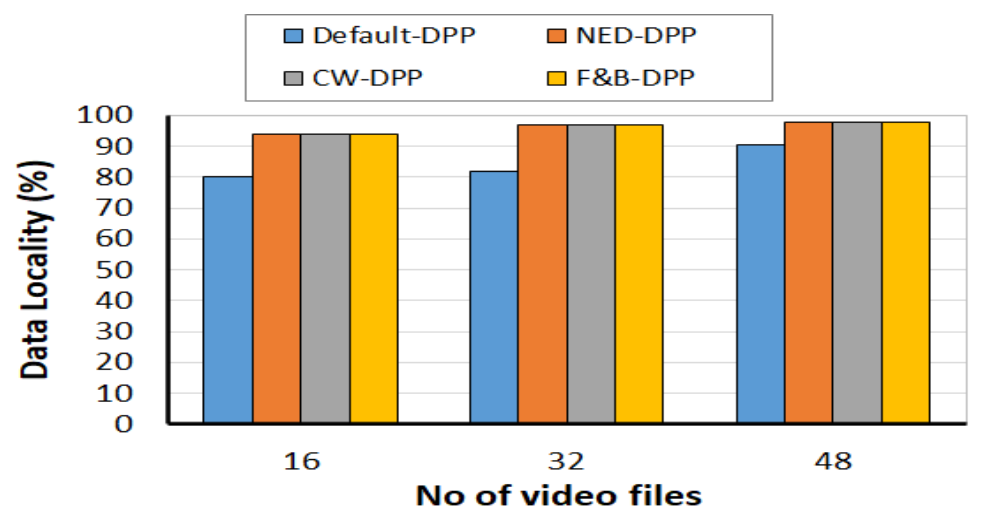

Figure 6.4: Increasing data size and locality (weekday).

In the weekend scenario, Figure 6.5 shows the superiority of CW-DPP and F\&BDPP over the other policies in terms of reducing the execution time of the ShdCD job, whatever the number of input video files. Table B.26 demonstrates the details of this experiment. The best performance is achieved when CW-DPP and F\&B-DPP were used.

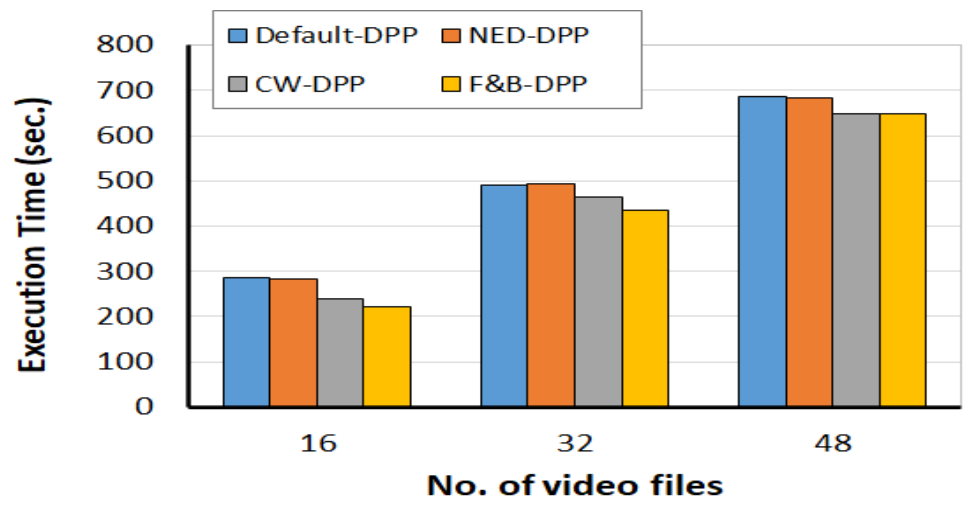

Figure 6.5: Increasing data size and execution time (weekend).

Figure 6.6 depicts the influence of the different policies on the locality under different input video files. The experimental results that show the effect of the replication factor on the execution time and locality of both SimpleCD and ShdCD are detailed in Table B.27. 


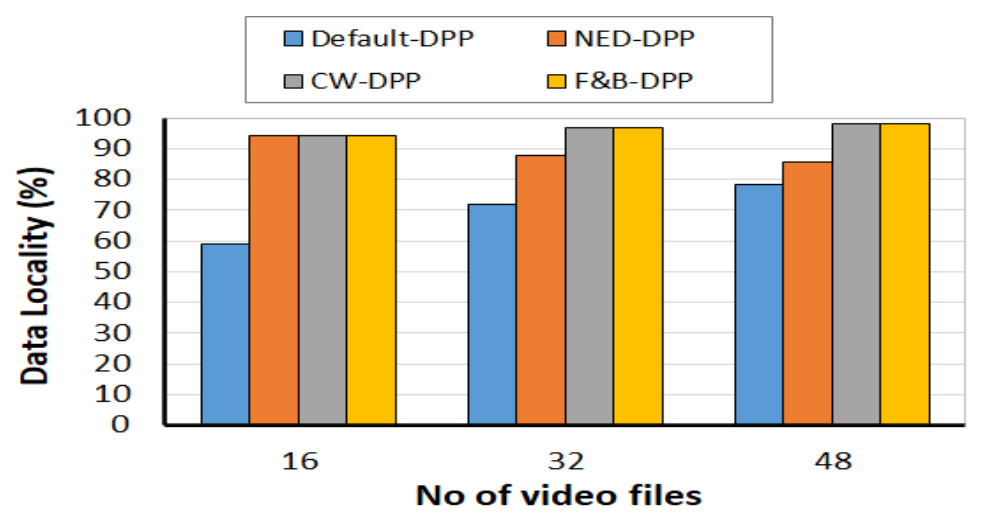

Figure 6.6: Increasing data size and locality (weekend).

Figure 6.7 reveals that the execution time of ShdCD improves when CW-DPP with one replica and F\&B-DPP with one replica are used to store the input video segments. This means that the proposed policies with one replica outperform Default - DPP with 3 replicas, hence resulting in more efficient performance. On the other hand, SimpleCD is not sensitive to any DPP since it doesn't generate different workloads as ShdCD does.

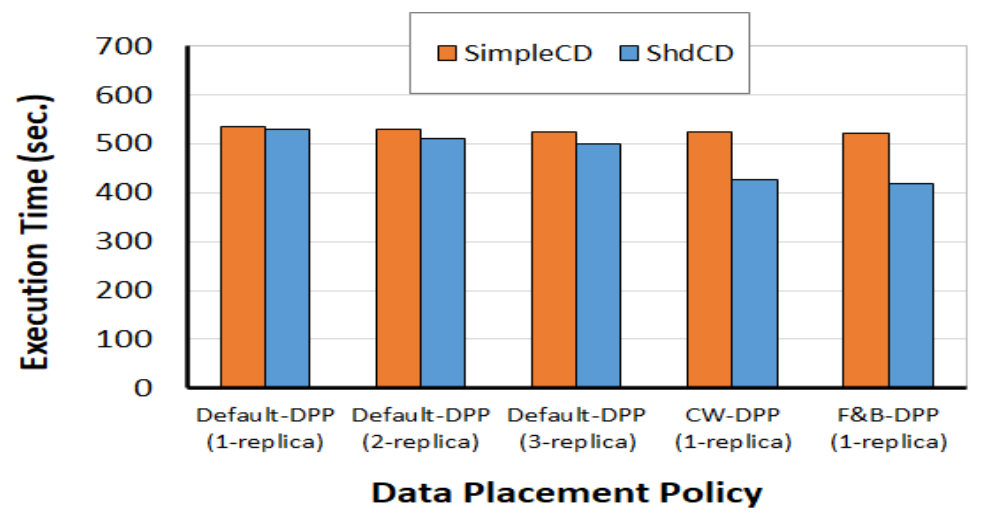

Figure 6.7: The effect of the no. of replicas on execution time.

Figure 6.8 reveals the influence of the replication factor on the locality. With less number of replicas, CW-DPP and F\&B-DPP outperform the performance of Default-DPP in terms of improving the locality for both SimpleCD and ShdCD. 


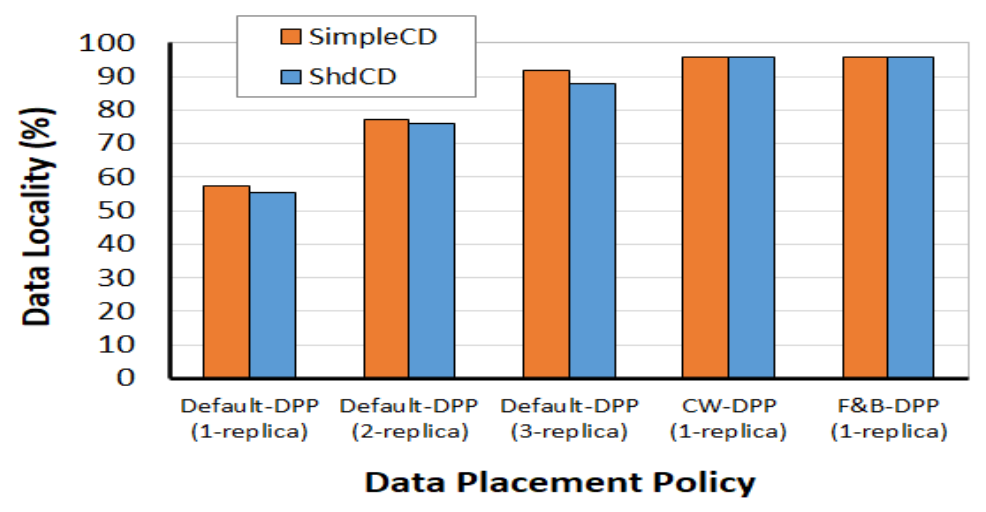

Figure 6.8: The effect of the no. of replicas on the Locality.

Figure 6.9 shows the influence of one-node failure on the execution time, where two replicas have been used for each video segment. There are no significant differences in the execution time since the size of the processed video data was not big enough, and the network speed on Cloud is fast enough to minimize the effect of transferring the data over the network.

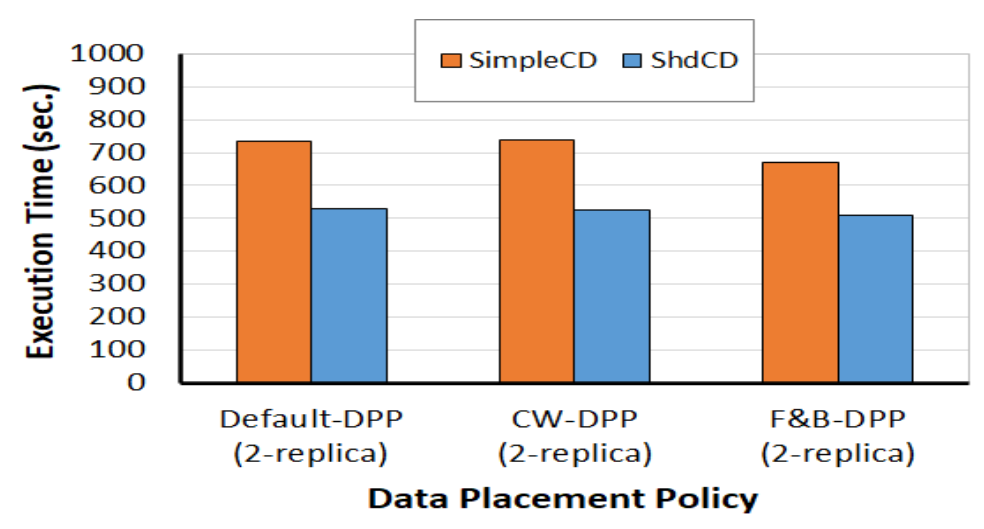

Figure 6.9: Effect of one-node failure on execution time.

Figure 6.10 shows how CW-DPP outperforms Default-DPP, and F\&B-DPP outperforms $C W-D P P$ in terms of improving the locality performance of both SimpleCD and ShdCD. Table B.28 lists the statistics of the effect of one-node failure on the execution time of SimpleCD and ShdCD when Default-DPP, 
F\&B-DPP, and CW-DPP have been used with two replicas to distribute the input video segments.

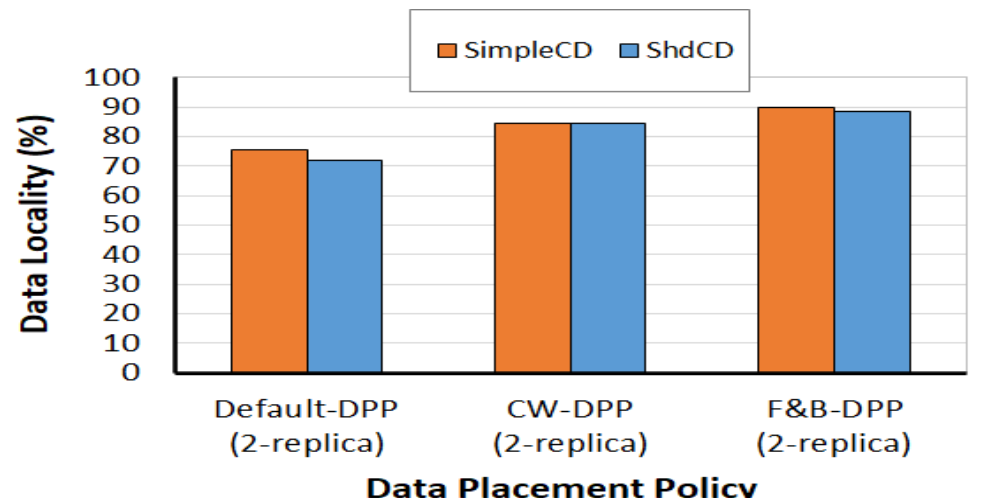

Figure 6.10: Effect of one-node failure on the locality.

Figure 6.11 shows the effect of two-node failure on the performance of DefaultDPP and ECW-DPP, where three replicas are used to support the recovery. As demonstrated in the figure, there is no significant difference between the two DPP.

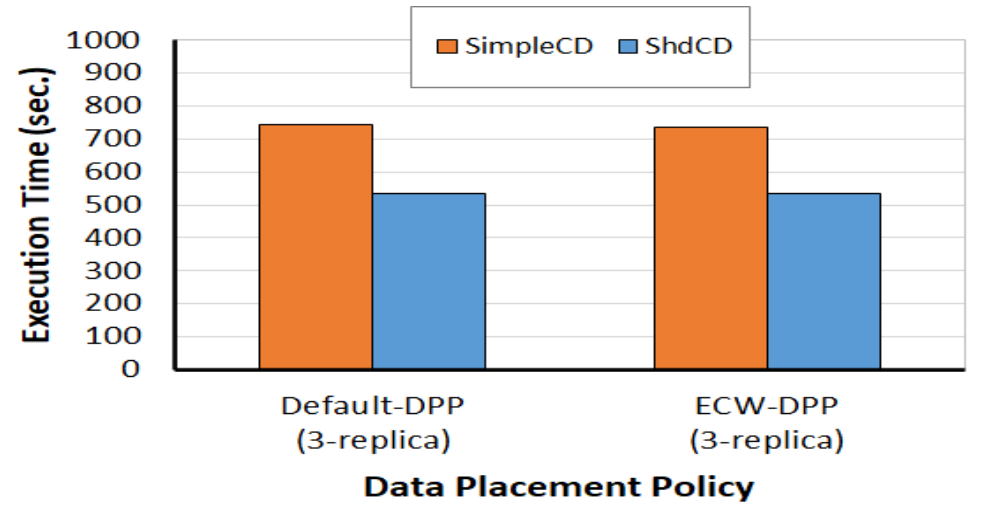

Figure 6.11: Effect of two-node failure on execution time.

Furthermore, Figure 6.12 reveals marginally locality performance for the interest of $E C W-D P P$. More details of the two-node failure test are demonstrated in Table B.29. In general, to support recovery from n-node failures, we need to maintain $n+1$ replicas for each video segment. Because of the cost and the time associated with 
running such experiments, only one-node and two-node failures have been considered.

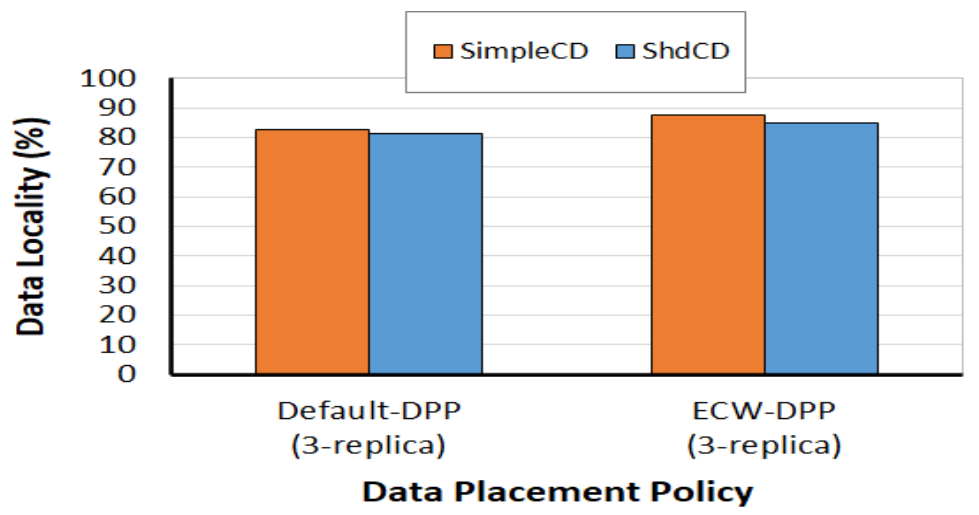

Figure 6.12: Effect of two-node failure on the locality.

Table 6.1 shows a summary of the performance of all the data placement policies discussed in this chapter. The summary uses the execution time and locality metrics to compare the policies with one another. The symbol '-' in the table denotes that the experiments have not been conducted for the following reasons. NED-DPP (the policy used in the literature to distribute video data) does not support fault tolerance. ECW-DPP is the extended version of CW-DPP to support recovery from two-node failures. F\&B-DPP supports two-node failures; however, the experiments were not conducted to prevent a further increase in the time associated with the empirical study.

\subsection{Chapter Summary}

This thesis presents an approach (ShdCD) to improve the execution time of MapReduce-based change detection using a sampling strategy. The goal of the sampling strategy is to reduce the workload of processing each video segment by avoiding unnecessary computation; however, this strategy leads to access load imbalance. To address this challenge, three data placement policies are devised mainly to minimize 
Table 6.1: Data placement policies summary

\begin{tabular}{|c|c|c|c|c|c|c|}
\hline \multirow{2}{*}{ Algorithm } & \multicolumn{6}{|c|}{ Performance } \\
\cline { 2 - 8 } & \multicolumn{2}{|c|}{$\begin{array}{c}\text { No failure } \\
\text { (one replica) }\end{array}$} & \multicolumn{2}{c|}{$\begin{array}{c}\text { One-node failure } \\
\text { (two replicas) }\end{array}$} & $\begin{array}{c}\text { Two-node failures } \\
\text { (three replicas) }\end{array}$ \\
\cline { 2 - 8 } & time & locality & time & locality & time & locality \\
\hline Default-DPP & longest & low & short & moderate & short & moderate \\
\hline NED-DPP & long & moderate & - & - & - & - \\
\hline CW-DPP & short & high & short & high & - & - \\
\hline ECW-DPP & - & - & - & - & short & moderate \\
\hline F\&B-DPP & shortest & high & shortest & highest & - & - \\
\hline
\end{tabular}

data migration across the network. The proposed approach, ShdCD, can be exploited in a wide range of video processing applications since, in this discipline, it is common to process only the region of interest rather than the entire video data. The experiments, conducted on Cloud Computing using Amazon EC2 instances, indicate that ShdCD saves up to $32 \%$ of Normal-MR execution time. Furthermore, CW-DPP shows performance that outmatches the performance of the default data placement policy in minimizing the data migration overhead. 


\section{Chapter 7}

\section{Conclusions and Future Work}

\subsection{Conclusions}

Video surveillance is an important technology that can be used in different applications such as tracking objects, monitoring people and traffic, and detecting anomalies. One of the most important methods in video processing is change detection since it can be used as an elementary step in many video processing applications. Extracting knowledge and useful information from large video data requires large media to store the data as well as significant computing capability. Apache Hadoop can meet this requirement through HDFS to store the video data in a distributed manner, and the MapReduce programming model to access the stored data and process them in parallel. Although using distributed computing to process large video data is beneficial, increasing the degree of parallelism incurs more overhead that limits the advantages.

This thesis introduces different algorithms to improve the performance of using CD to process big video data on Hadoop clusters. In Chapter 3, two algorithms have been introduced to split large video sequences before storing the data in HDFS. The experimental results show how the way used to split the video data can improve

the performance by reducing the execution time. In Chapter 4, a sampling-based 
approach is devised to avoid unnecessary computation in order to minimize the execution time. In fact, not all frames are required to be processed while processing a video sequence. This is because there may be no significant changes in the scene and hence processing similar frames wastes the resources. The experimental results show how avoiding unnecessary computation improves the processing time. Chapter 5 describes different sampling techniques to improve the performance of MapReducebased CD by avoiding unnecessary computation. Different experiments have been conducted to show how the proposed algorithms can improve the performance in

fault-tolerant systems. The experimental results show that the proposed sampling techniques can improve the execution time when faults occur on the system. Chapter 6 describes how the proposed DPPs can play a significant role in improving the performance of MapReduce-based CD. The importance of DPP is that it can balance the workload among the nodes of a Hadoop cluster, and minimize the network overhead. The experimental results show how the proposed DPPs improve the performance of running MapReduce-based CD by reducing the execution time.

\subsection{Future Work}

This thesis proposes a video splitting strategy that supports running change detection that requires overlapping some video frames to make the distributed processing feasible. Since overlapping the frames is considered overhead, the proposed solution is directed at minimizing this overhead by sampling the history frames of each segment. This thesis uses two different patterns to sample the overlapping frames (e.g., the Fibonacci scheme and strides); for future work, more patterns can be investigated. Furthermore, since the MapReduce programming model has been designed to process big data, the goal is to perform more experiments using large video data taken from different scenes to investigate the effect of the proposed algorithms on 
different scenarios. Moreover, the proposed splitting mechanisms are not restricted to the Hadoop based MapReduce programming model. The possibility of adapting these mechanisms to the Apache Spark framework that is based on the in-memory processing concept can be investigated.

The thesis also introduces approaches that rely on using the MapReduce programming model to process the video data in parallel and using a sampling method to reduce the computation on each node (Chapter 5). In this regard, more video data can be considered from different scenarios (other than the skating scenario). Apache Spark could be used to implement the proposed sampling-based change detection since the proposed solutions are generic and not confined to the MapReduce programming model. The proposed sampling-based CD could be used to improve the performance of face recognition and mobile object tracking applications. In video surveillance, face recognition can be used in suspect detection applications. The challenge is that a large number of video frames need to be processed to detect suspects. By using the proposed sampling-based CD, the processing will be confined to some frames and part of the selected frames (RoI) to reduce the computation time. A mobile object tracking system can be developed as future work. Mobile object tracking can benefit from the proposed sampling-based techniques to reduce computation time. This can be achieved by restricting the computation to merely include RoI.

Finally, Chapter 6 introduces three Data placement policies to alleviate the negative effect of the sampling-based MapReduce CD. Different scenarios will be recorded and investigated to give more insights into the applicability of the proposed DPPs. Further, larger video data and larger clusters need to be experimented to investigate the scalability of the proposed algorithms. The impact of the proposed DPPs on the execution time of different applications such as face detection and mobile object tracking that depend on the proposed sampling-based CD will be investigated. 


\section{List of References}

[1] H. J. Chang, H. Jeong, and J. Y. Choi, "Active attentional sampling for speedup of background subtraction," in IEEE Conference on Computer Vision and Pattern Recognition, (Providence, RI, USA), 2012.

[2] Z. Zivkovic, "Improved adaptive gaussian mixture model for background subtraction," in International Conference on Pattern Recognition, ICPR 2004., (Cambridge, UK), 2004.

[3] C. Stauffer and W. E. L. Grimson, "Adaptive background mixture models for real-time tracking," in IEEE Computer Society Conference on Computer Vision and Pattern Recognition, (Fort Collins, CO, USA), 1999.

[4] P. K. Atrey, V. Kumar, A. Kumar, and M. S. Kankanhalli, "Experiential sampling based foreground/background segmentation for video surveillance," in IEEE International Conference on Multimedia and Expo, (Toronto, ON, Canada), 2006.

[5] D. Lee, J. Ahn, and C. Kim, "Fast background subtraction algorithm using twolevel sampling and silhouette detection," in IEEE International Conference on Image Processing (ICIP), (Cairo, Egypt), 2009.

[6] Z. Tang and Z. Miao, "Fast background subtraction using improved gmm and graph cut," in Congress on Image and Signal Processing, (Sanya, Hainan, China), 2008.

[7] L. Mabrouk, S. Huet, D. Houzet, S. Belkouch, A. Hamzaoui, and Y. Zennayi, "Efficient parallelization of gmm background subtraction algorithm on a multi-core platform for moving objects detection," in International Conference on Advanced Technologies for Signal and Image Processing (ATSIP), (Sousse, Tunisia), 2018.

[8] C. Zhang, H. Tabkhi, and G. Schirner, "A gpu-based algorithm-specific optimization for high-performance background subtraction," in International Conference on Parallel Processing, (Minneapolis MN, USA), 2014. 
[9] V. Pham, P. Vo, V. T. Hung, and L. H. Bac, "Gpu implementation of extended gaussian mixture model for background subtraction," in IEEE RIVF International Conference on Computing Communication Technologies, Research, Innovation, and Vision for the Future (RIVF), (Hanoi, Vietnam), 2010.

[10] X. Ye and W. Wan, "Fast background modeling using gmm on gpu," in International Conference on Audio, Language and Image Processing, (Shanghai, China), 2014.

[11] P. Gudivaka, N. Mishra, and A. Agrawal, "Gpu accelerated foreground segmentation using codebook model and shadow removal using cuda," in International Conference on Computing, Communication and Automation (ICCCA), (Greater Noida, India), 2017.

[12] M. M. Mubasher, M. S. Farid, A. Khaliq, and M. M. Yousaf, "A parallel algorithm for change detection," in International Multitopic Conference (INMIC), (Islamabad, Pakistan), 2012.

[13] "Hadoop." http://hadoop.apache.org. Accessed: Jul 11, 2020.

[14] T. White, Hadoop: The Definitive Guide. O'Reilly Media, Inc., 4th ed., 2015.

[15] I. A. T. Hashem, I. Yaqoob, N. B. Anuar, S. Mokhtar, A. Gani, and S. U. Khan, "The rise of big data on cloud computing: Review and open research issues," Information systems, vol. 47, 2015.

[16] H. Tan and L. Chen, "An approach for fast and parallel video processing on apache hadoop clusters," in IEEE International Conference on Multimedia and Expo (ICME), (Chengdu, China), 2014.

[17] X. Zhao, H. Ma, H. Zhang, Y. Tang, and G. Fu, "Metadata extraction and correction for large-scale traffic surveillance videos," in IEEE International Conference on Big Data (Big Data), (Washington, DC, USA), 2014.

[18] H. H. Wu, T. C. Yeh, and C. M. Wang, "Multiple two-phase data processing with mapreduce," in IEEE International Conference on Cloud Computing, (Anchorage, AK, USA), 2014.

[19] X. Liu, D. Zhao, L. Xu, W. Zhang, J. Yin, and X. Chen, "A distributed video management cloud platform using hadoop," IEEE Access, vol. 3, 2015. 
[20] R. Zheng, L. Chen, H. Jin, L. Zhu, and Q. Zhang, "A novel metadata extraction method for surveillance video," in International Conference on Computing, Networking and Communications (ICNC), (Kauai, HI, USA), 2016.

[21] N. Goyette, P. Jodoin, F. Porikli, J. Konrad, and P. Ishwar, "Changedetection.net: A new change detection benchmark dataset," in IEEE Computer Society Conference on Computer Vision and Pattern Recognition Workshops, (Providence, RI, USA), 2012.

[22] "Ffmpeg." https://www.ffmpeg.org. Accessed: Jul 11, 2020.

[23] T. Bouwmans, L. Maddalena, and A. Petrosino, "Scene background initialization: A taxonomy," Pattern Recognition Letters, vol. 96, 2017.

[24] M. Alexa, "Extracting the essence from sets of images," in Eurographics Conference on Computational Aesthetics in Graphics, Visualization and Imaging, (Banff, Alberta, Canada), 2007.

[25] S. Amri, W. Barhoumi, and E. Zagrouba, "Unsupervised background reconstruction based on iterative median blending and spatial segmentation," in IEEE International Conference on Imaging Systems and Techniques, (Thessaloniki, Greece), 2010.

[26] Eng, Toh, Kam, Wang, and Yau, "An automatic drowning detection surveillance system for challenging outdoor pool environments," in IEEE International Conference on Computer Vision, (Nice, France), 2003.

[27] Y. Liu, H. Yao, W. Gao, X. Chen, and D. Zhao, "Nonparametric background generation," Journal of Visual Communication and Image Representation, vol. 18, 2007.

[28] M. Molinier, T. Häme, e. H. Ahola, Heikki", J. Parkkinen, and A. Kaarna, "3dconnected components analysis for traffic monitoring in image sequences acquired from a helicopter," in Image Analysis, (Berlin, Heidelberg), 2005.

[29] P. Roth, H. Bischof, D. Skocaj, and A. Leonardis, "Object detection with bootstrapped learning," in Computer Vison Winter workshop, (Styria, Austria), 2005.

[30] P. Abu, V. S. Chu, and P. Fernandez, "A monte-carlo-based algorithm for background generation," Philippine Information Technology Journal, vol. 6, 2013.

[31] P. F. Kardi Teknomo, "Background image generation using boolean operations," Philippine Computing Journal, vol. 4, 2009. 
[32] A. Elgammal, D. Harwood, and L. Davis, "Non-parametric model for background subtraction," in European conference on computer vision, (Berlin, Heidelberg), 2000 .

[33] C. Chen and J. K. Aggarwal, "An adaptive background model initialization algorithm with objects moving at different depths," in IEEE International Conference on Image Processing, (San Diego, CA, USA), 2008.

[34] C. Chiu, M. Ku, and L. Liang, "A robust object segmentation system using a probability-based background extraction algorithm," IEEE Transactions on Circuits and Systems for Video Technology, vol. 20, 2010.

[35] R. Zhang, W. Gong, A. Yaworski, and M. Greenspan, "Nonparametric on-line background generation for surveillance video," in International Conference on Pattern Recognition (ICPR2012), (Tsukuba, Japan), 2012.

[36] H.-H. Hsiao and J.-J. Leou, "Background initialization and foreground segmentation for bootstrapping video sequences," EURASIP Journal on Image and Video Processing, vol. 2013, 2013.

[37] D. Ortego, J. C. SanMiguel, and J. M. Martínez, "Rejection based multipath reconstruction for background estimation in video sequences with stationary objects," Computer Vision and Image Understanding, vol. 147, 2016.

[38] V. Reddy, C. Sanderson, and B. C. Lovell, "A low-complexity algorithm for static background estimation from cluttered image sequences in surveillance contexts," EURASIP Journal on Image and Video Processing, vol. 2011, 2010.

[39] Y. Nakashima, N. Babaguchi, and J. Fan, "Automatic generation of privacyprotected videos using background estimation," in IEEE International Conference on Multimedia and Expo, (Barcelona, Spain), 2011.

[40] D. Park and H. Byun, "A unified approach to background adaptation and initialization in public scenes," Pattern Recognition, vol. 46, 2013.

[41] A. Shrotre and L. J. Karam, "Background recovery from multiple images," in IEEE Digital Signal Processing and Signal Processing Education Meeting (DSP/SPE), (Napa, CA, USA), 2013.

[42] A. Colombari, M. Cristani, V. Murino, and A. Fusiello, "Exemplar-based background model initialization," in ACM International Workshop on Video Surveillance and Sensor Networks, (Singapore), 2005. 
[43] A. Sobral, T. Bouwmans, e. V. Zahzah, El-hadi", E. Puppo, D. Sona, M. Cristani, and C. Sansone, "Comparison of matrix completion algorithms for background initialization in videos," in New Trends in Image Analysis and Processing ICIAP 2015 Workshops, (Genoa, Italy), 2015.

[44] A. Sobral and E. hadi Zahzah, "Matrix and tensor completion algorithms for background model initialization: A comparative evaluation," Pattern Recognition Letters, vol. 96, 2017.

[45] M. D. Gregorio and M. Giordano, "Background estimation by weightless neural networks," Pattern Recognition Letters, vol. 96, 2017.

[46] L. Maddalena and A. Petrosino, "The sobs algorithm: What are the limits?," in IEEE Computer Society Conference on Computer Vision and Pattern Recognition Workshops, (Providence, RI, USA), 2012.

[47] J. A. Ramirez-Quintana and M. I. Chacon-Murguia, "Self-adaptive som-cnn neural system for dynamic object detection in normal and complex scenarios," Pattern Recognition, vol. 48, 2015.

[48] M. Braham and M. Van Droogenbroeck, "Deep background subtraction with scene-specific convolutional neural networks," in International Conference on Systems, Signals and Image Processing (IWSSIP), (Bratislava, Slovakia), 2016.

[49] Y. Wang, Z. Luo, and P.-M. Jodoin, "Interactive deep learning method for segmenting moving objects," Pattern Recognition Letters, vol. 96, 2017.

[50] Z. Zhao, X. Zhang, and Y. Fang, "Stacked multilayer self-organizing map for background modeling," IEEE Transactions on Image Processing, vol. 24, 2015.

[51] A. Bevilacqua, L. Di Stefano, and A. Lanza, "An effective multi-stage background generation algorithm," in IEEE Conference on Advanced Video and Signal Based Surveillance, 2005., (Como, Italy), 2005.

[52] H. Wang, e.-P. J. Suter, David", S. K. Nayar, and H.-Y. Shum, "A novel robust statistical method for background initialization and visual surveillance," in Computer Vision - ACCV 2006, (Berlin, Heidelberg), 2006.

[53] A. Colombari and A. Fusiello, "Patch-based background initialization in heavily cluttered video," IEEE Transactions on Image Processing, vol. 19, 2010. 
[54] X. Chen, Y. Zhu, Y. Guo, B. Zhou, X. Zhao, Y. Du, H. Lei, M. Li, and Z. Wang, "Carbonization synthesis of hydrophobic caco3 at room temperature," Colloids and Surfaces A: Physicochemical and Engineering Aspects, vol. 353, 2010.

[55] Xun Xu and T. S. Huang, "A loopy belief propagation approach for robust background estimation," in IEEE Conference on Computer Vision and Pattern Recognition, (Anchorage, AK, USA), 2008.

[56] H. Lin, T. Liu, and J. Chuang, "Learning a scene background model via classification," IEEE Transactions on Signal Processing, vol. 57, 2009.

[57] L. Maddalena and A. Petrosino, "A self-organizing approach to background subtraction for visual surveillance applications," IEEE Transactions on Image Processing, vol. 17, 2008.

[58] B. Garcia-Garcia, T. Bouwmans, and A. J. R. Silva, "Background subtraction in real applications: Challenges, current models and future directions," Computer Science Review, vol. 35, 2020.

[59] R. A. Hadi, L. E. George, and M. J. Mohammed, "A computationally economic novel approach for real-time moving multi-vehicle detection and tracking toward efficient traffic surveillance," Arabian Journal for Science and Engineering, vol. $42,2017$.

[60] K. Williams, R. Towler, and C. Wilson, "Cam-trawl: a combination trawl and stereo-camera system," Sea Technology, vol. 51, 2010.

[61] S. Muniruzzaman, N. Haque, F. Rahman, M. Siam, R. Musabbir, M. Hadiuzzaman, and S. Hossain, "Deterministic algorithm for traffic detection in free-flow and congestion using video sensor," Journal of Built Environment, Technology and Engineering, vol. 1, 2016.

[62] A. Leykin and M. Tuceryan, "Detecting shopper groups in video sequences," in IEEE Conference on Advanced Video and Signal Based Surveillance, (London, UK), 2007.

[63] N. Avinash, M. S. Kumar, and S. Sagar, "Automated video surveillance for retail store statistics generation," in International Conference on Signal and Image Processing 2012 (ICSIP 2012), (India), 2013.

[64] U. Knauer, M. Himmelsbach, F. Winkler, F. Zautke, K. Bienefeld, and B. Meffert, "Application of an adaptive background model for monitoring honeybees," 
in IASTED International Conference on Visualization, Imaging and Image Processing, (Benidorm, Spain), 2005.

[65] B. Rezaei and S. Ostadabbas, "Moving object detection through robust matrix completion augmented with objectness," IEEE Journal of Selected Topics in Signal Processing, vol. 12, 2018.

[66] M. Ashraphijuo, V. Aggarwal, and X. Wang, "On deterministic sampling patterns for robust low-rank matrix completion," IEEE Signal Processing Letters, vol. 25, 2017.

[67] P. Dickinson, R. Freeman, S. Patrick, S. Lawson, et al., "Autonomous monitoring of cliff nesting seabirds using computer vision," in International Workshop on Distributed Sensing and Collective Intelligence in Biodiversity Monitoring, (Amsterdam, Netherlands), 2008.

[68] P. Dickinson, C. Qing, S. Lawson, R. Freeman, et al., "Automated visual monitoring of nesting seabirds," in Workshop on visual observation and analysis of animal and insect behaviour, (Istanbul, Turkey), 2010.

[69] C. J. Cohen, D. Haanpaa, and J. P. Zott, "Machine vision algorithms for robust animal species identification," in IEEE Applied Imagery Pattern Recognition Workshop (AIPR), (Washington, DC, USA), 2015.

[70] C. J. Cohen, D. Haanpaa, S. Rowe, and J. P. Zott, "Vision algorithms for automated census of animals," in IEEE Applied Imagery Pattern Recognition Workshop (AIPR), (Washington, DC, USA), 2011.

[71] A. Borghgraef, O. Barnich, F. Lapierre, M. Van Droogenbroeck, W. Philips, and M. Acheroy, "An evaluation of pixel-based methods for the detection of floating objects on the sea surface," EURASIP Journal on Advances in Signal Processing, vol. 2010, 2010.

[72] B. Tamás, "Detecting and analyzing rowing motion in videos," in BME Scientific Student Conference, (Budapest, Hungary), 2016.

[73] G. Tzanidou, Carried baggage detection and recognition in video surveillance with foreground segmentation. Department of computer science, Loughborough University, 2014.

[74] B. U. Töreyin, Y. Dedeoğlu, U. Güdükbay, and A. E. Cetin, "Computer vision based method for real-time fire and flame detection," Pattern recognition letters, vol. 27, 2006. 
[75] C. Lee, Y. Terada, Y. Liu, and B. Lee, "A motion accuracy evaluator based on body parts movement by mapreduce video processing," in IEEE-EMBS International Conference on Biomedical and Health Informatics (BHI), (Valencia, Spain), 2014.

[76] G. Li, X. Li, F. Yang, J. Teng, S. Ding, Y. F. Zheng, D. Xuan, B. Chen, and W. Zhao, "Traffic at-a-glance: Time-bounded analytics on large visual traffic data," IEEE Transactions on Parallel and Distributed Systems, vol. 28, 2017.

[77] C. Ryu, D. Lee, M. Jang, C. Kim, and E. Seo, "Extensible video processing framework in apache hadoop," in IEEE international conference on cloud computing technology and science, (Bristol, UK), 2013.

[78] M. Y. Eltabakh, Y. Tian, F. Özcan, R. Gemulla, A. Krettek, and J. McPherson, "Cohadoop: Flexible data placement and its exploitation in hadoop," VLDB Endowment, vol. 4, 2011.

[79] X. Hou, A. K. T. K, J. P. Thomas, and V. Varadharajan, "Dynamic workload balancing for hadoop mapreduce," in IEEE International Conference on Big Data and Cloud Computing, (Sydney, NSW, Australia), 2014.

[80] Jiong Xie, Shu Yin, Xiaojun Ruan, Zhiyang Ding, Yun Tian, J. Majors, A. Manzanares, and Xiao Qin, "Improving mapreduce performance through data placement in heterogeneous hadoop clusters," in IEEE International Symposium on Parallel Distributed Processing, Workshops and Phd Forum (IPDPSW), (Atlanta, GA, USA), 2010.

[81] H. Hsiao, H. Chung, H. Shen, and Y. Chao, "Load rebalancing for distributed file systems in clouds," IEEE Transactions on Parallel and Distributed Systems, vol. 24, 2013.

[82] C. Lin and Y. Lin, "A load-balancing algorithm for hadoop distributed file system," in International Conference on Network-Based Information Systems, (Taipei, Taiwan), 2015.

[83] W. Dai, I. Ibrahim, and M. Bassiouni, "A new replica placement policy for hadoop distributed file system," in IEEE International Conference on Big Data Security on Cloud (BigDataSecurity), IEEE International Conference on High Performance and Smart Computing (HPSC), and IEEE International Conference on Intelligent Data and Security (IDS), (New York, NY, USA), 2016. 
[84] W. Dai, I. Ibrahim, and M. Bassiouni, "An improved replica placement policy for hadoop distributed file system running on cloud platforms," in IEEE International Conference on Cyber Security and Cloud Computing (CSCloud), (New York, NY, USA), 2017.

[85] J. Dharanipragada, S. Padala, B. Kammili, and V. Kumar, "Tula: A disk latency aware balancing and block placement strategy for hadoop," in IEEE International Conference on Big Data (Big Data), (Boston, MA, USA), 2017.

[86] S. M. Shithil, T. K. Saha, and T. Sharma, "A dynamic data placement policy for heterogeneous hadoop cluster," in International Conference on Advances in Electrical Engineering (ICAEE), (Dhaka, Bangladesh), 2017.

[87] J. Liao, Z. Cai, F. Trahay, and X. Peng, "Block placement in distributed file systems based on block access frequency," IEEE Access, vol. 6, 2018.

[88] A. Shah and M. Padole, "Load balancing through block rearrangement policy for hadoop heterogeneous cluster," in International Conference on Advances in Computing, Communications and Informatics (ICACCI), (Bangalore, India), 2018.

[89] H. Zhang, B. Xu, J. Yan, L. Liu, and H. Ma, "Proactive data placement for surveillance video processing in heterogeneous cluster," in IEEE International Conference on Cloud Computing Technology and Science (CloudCom), (Luxembourg City, Luxembourg), 2016.

[90] A. Heikkinen, J. Sarvanko, M. Rautiainen, and M. Ylianttila, "Distributed multimedia content analysis with mapreduce," in IEEE Annual International Symposium on Personal, Indoor, and Mobile Radio Communications (PIMRC), (London, UK), 2013.

[91] Chen Liu, Kun Fan, Zengqiu Yang, and Jiapeng Xiu, "A distributed video share system based on hadoop," in IEEE International Conference on Cloud Computing and Intelligence Systems, (Shenzhen, China), 2014.

[92] Jia Li, Kunhui Lin, and Jingjin Wang, "Design of the mass multimedia files storage architecture based on hadoop," in International Conference on Computer Science Education, (Colombo, Sri Lanka), 2013.

[93] "Gimp." https://www.gimp.org/. Accessed: Jul 30, 2020.

[94] "Amazon web services (aws)." https://aws.amazon.com/. Accessed: Jul 30, 2020 . 
[95] "Open java development kit (openjdk)." https://openjdk.java.net/. Accessed: Jul 30, 2020. 


\section{Appendix A}

\section{Algorithms Details}

\section{A.1 SVSwS Algorithm}

SVSwS considers every $i^{\text {th }}$ frame (stride) from $V_{h}^{j}$ into $V_{h}^{\prime j}$ for each segment $j$. As shown in Algorithm 14, the loop starting in line 4 extracts the data part $V_{d}^{j}$ for each segment $j$. The loop spanning line 8-14 is used to determines the training set $V_{h}^{j}$, while the inner loop starting in line 10 generates the sample training set, $V_{h}^{\prime j}$. Finally, the loop starting in line 15 appends the sample training set to the data frames for each segment before returning all segments.

\section{A.2 SVSwFW Algorithm}

Algorithm 15 describes SVSwFW in some details. The loop starting in line 3 divides the data frames among the segments. The loop starting in line 9 generates the sample training set $V_{h^{\prime}}^{j}$. Line 10 extracts the training set $V_{h}^{j}$. The inner loop starting in line 11 slides the window over $V_{h}^{j}$, while the innermost loop beginning in line 12 samples the frames that match the value '1' in the window from $V_{h}^{j}$ into $V_{h^{\prime}}^{j}$. The loop starting in line 23 constructs all video segments by concatenating $V_{h^{\prime}}^{j}$ to $V_{d}^{j}$. Finally, the algorithm returns all video segments (line 26). 


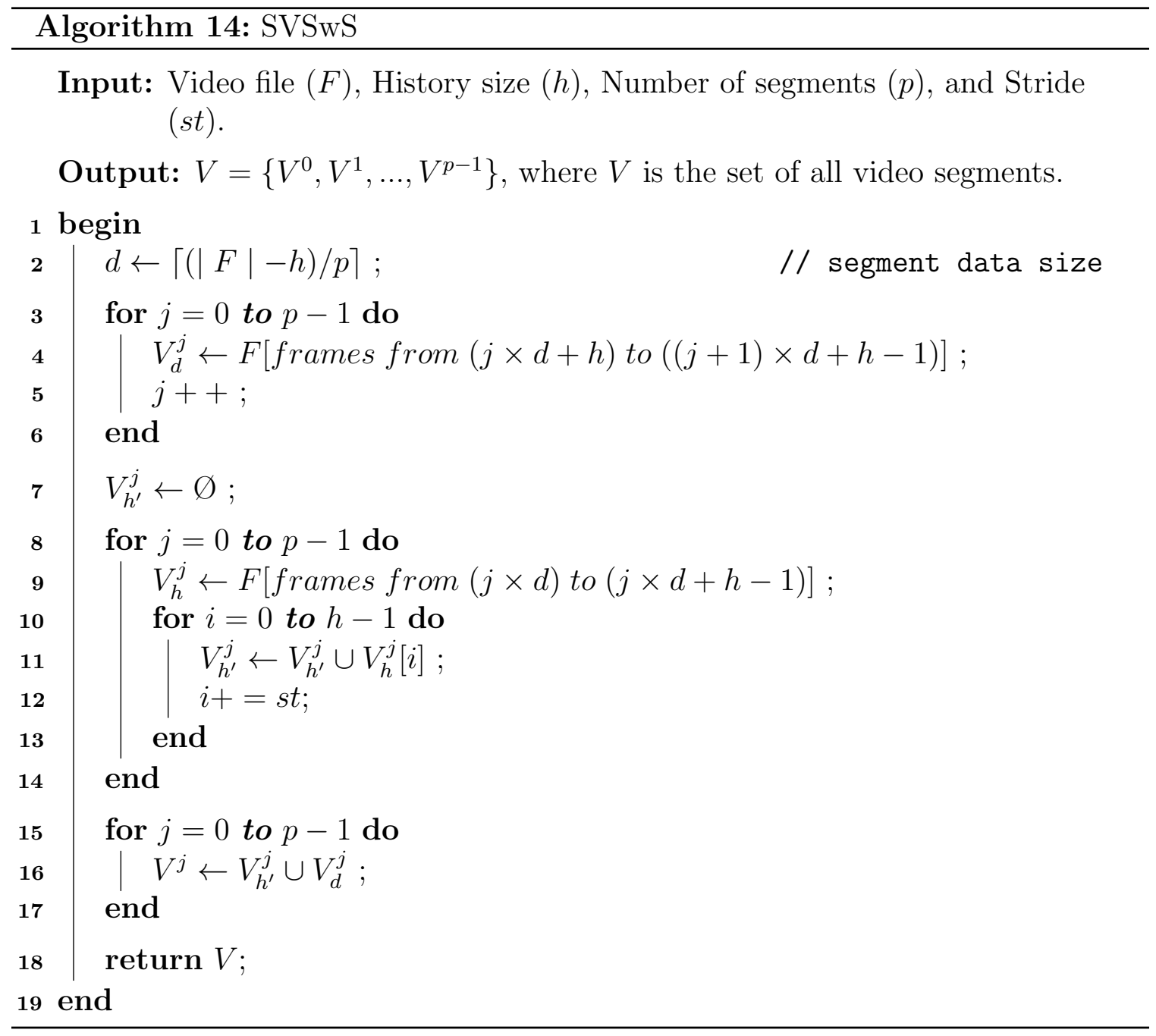

\section{A.3 Sampling-based background model update}

Algorithm 16 describes the proposed sampling method in more detail. The loop (Lines 7-23) iterates over the data frames $\left\{f_{d}^{t}: 0 \leq t<d\right\}$ to compute the binary mask frames $\left\{f_{b m}^{t}: 0 \leq t<d\right\}$ that signify the change detection. Line 9 detects the changes in the data frame at time $t$. The statements (Line 10-18) are to determine at the beginning of each window whether to sample through the current window or not. If AoC (calculated in Line 11) is larger than the high threshold $T_{h}$ (Lines 12-14), the background model will be updated with each data frame belonging to the current 


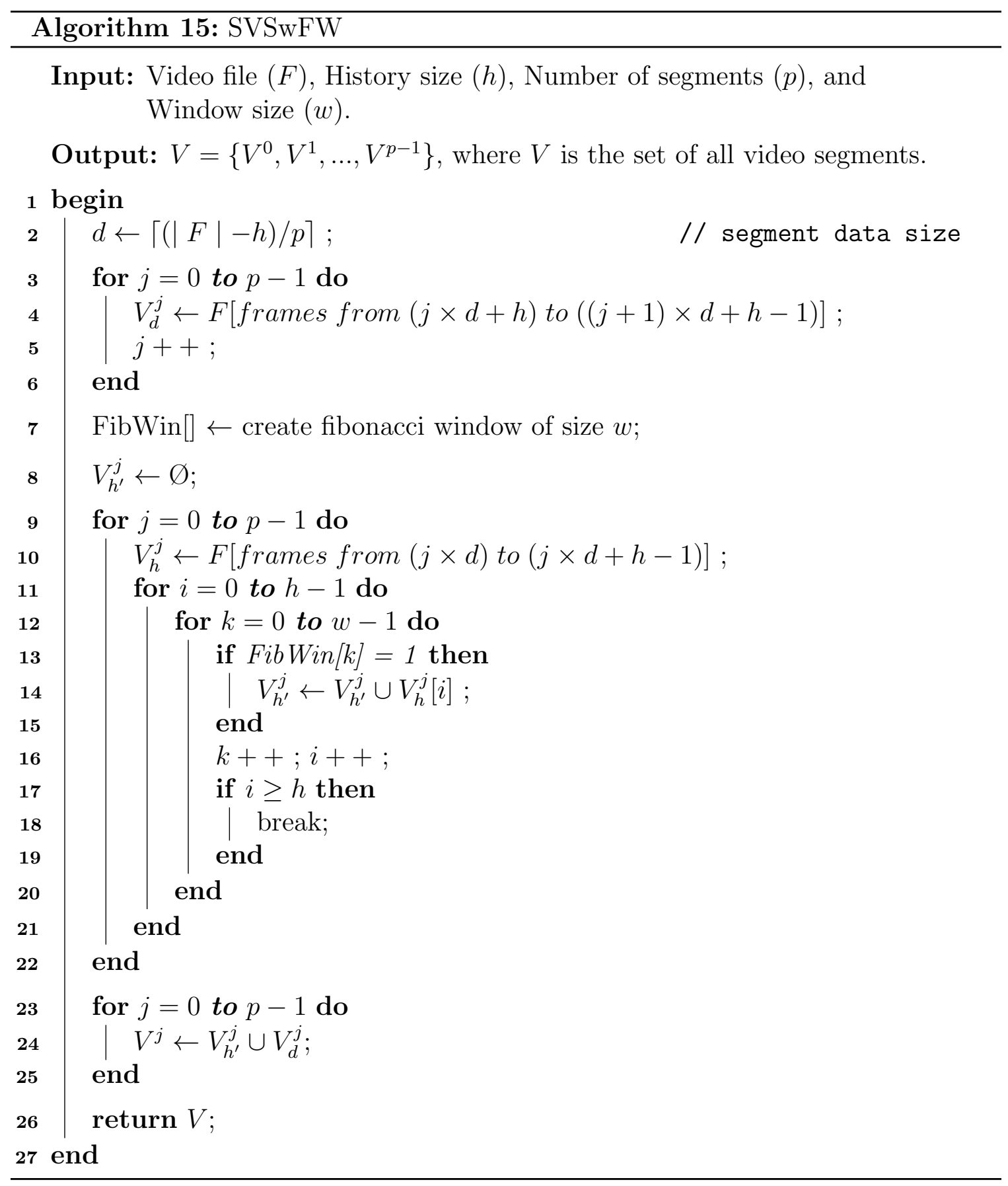


window. If AoC is smaller than the low threshold $T_{l}$ (Lines 15-17), the background model will be updated every $i^{t h}$ frame. The background model is updated based on the previously made decision (Lines 19-21). The set of all binary mask frames will be returned as output (Line 24).

\section{A.4 CW-DPP Algorithm}

Algorithm 17 describes how CW-DPP stores the data blocks/replicas of a given related dataset $\mathcal{V}$ in a set of datanodes $\mathcal{N}$. The output of the algorithm is to assign the replicas to the datanodes. The algorithm works with 1 or 2 replicas (Lines 2-4), so it does not waste resources especially in Cloud Computing. Initially, $U_{0}$ and $U_{1}$ update and sort the belonging datanodes information in non-decreasing order based on the load values (Line 8). After that, the algorithm decides which group to start placing the replicas in by comparing the first element in each utilization list. The selected group is the one that its first element has the smallest load value as shown in Lines 9-13. The algorithm runs in multiple rounds such that in each round $r$, it distributes $g$ replicas among the datanodes of a particular group before it switches to the other group in the following round $r+1$ to distribute other $g$ replicas among the datanodes of the current group, and so on. Within a group, the first replicas of $g$ video segments are assigned to the datanodes based on the datanodes order in the group utilization list. In case of the replication factor is 2 , the second replicas of the same video segments are assigned to the datanodes of the other group such that if the first replica is stored in a datanode of index $[i]$, the second replica will be stored in a datanode of index $[(1-k) g+(i+r) \bmod g]$ (Lines 19-23). The value $k$ denotes the current group, $g$ is the group size, $i$ is the index of the datanode in which the first replica is stored, and $r$ refers to the current round. 
Algorithm 16: Sampling-based background model update

Input: Video segment $\left(V^{j}\right)$, training set size $(h)$, high threshold $\left(T_{h}\right)$, low threshold $\left(T_{l}\right)$, and, initial background model $\left(f_{b g}^{0}\right)$.

Output: The set of all binary mask frames detecting the changes $\left(V_{b m}^{j}\right)$.

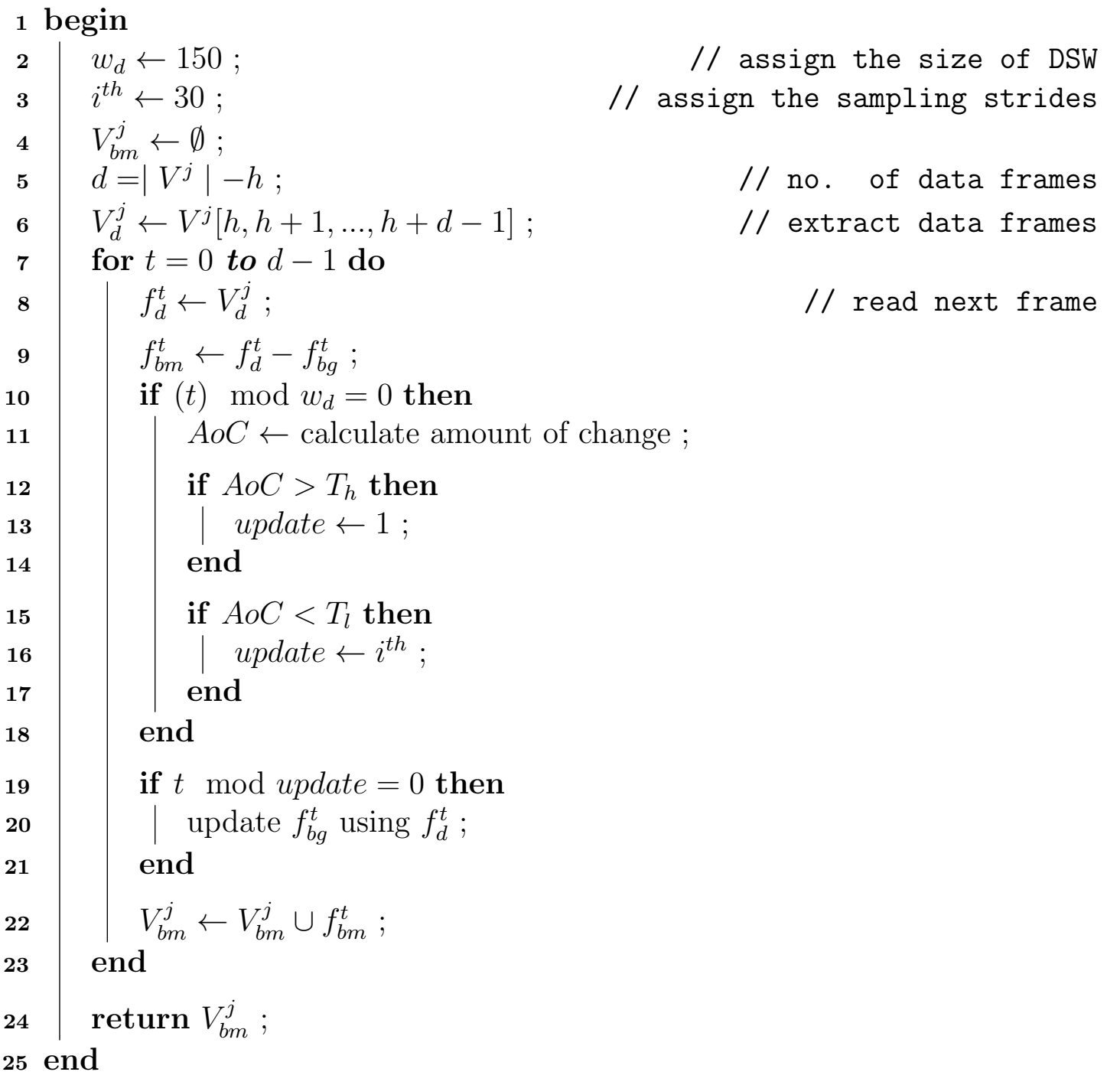


Algorithm 17: CW-DPP Algorithm

Input: Set of related video segment files $\mathcal{V}=\left\{V^{0}, V^{1}, \ldots, V^{s-1}\right\}$, Set of datanodes $\mathcal{N}=\left\{N_{0}, N_{1}, \ldots, N_{n-1}\right\}$, replication-factor .

Output: Data blocks assignmnet to datanodes.

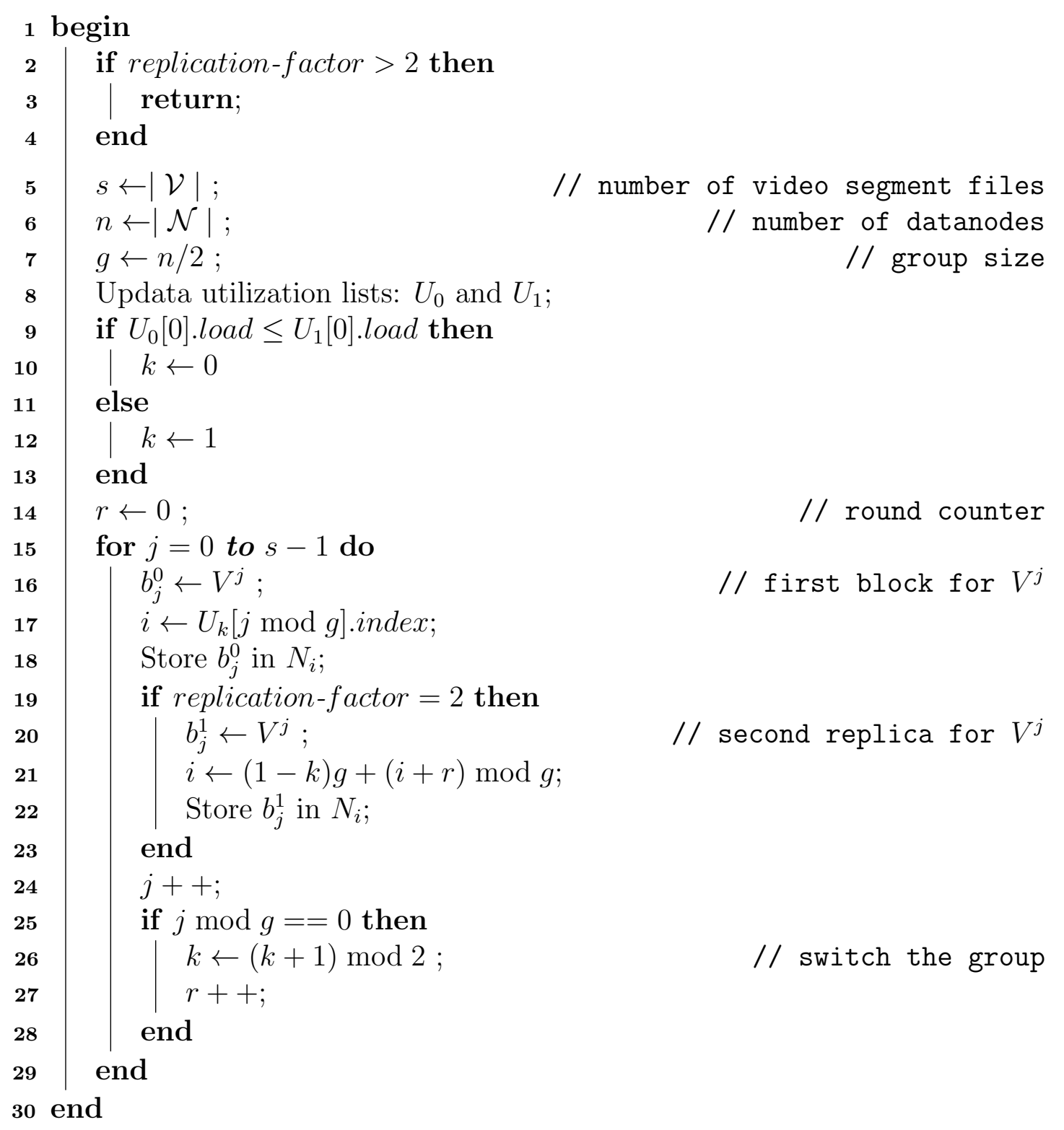




\section{Appendix B}

\section{Experimental Results}

This appendix provides the details of the experiments conducted for this thesis research. The details include the different parameters used and their effects on the accuracy and performance measurements.

\section{B.1 The Video Splitting Approach}

This section shows the statistics of the proposed video splitting techniques and their effects on the accuracy (Chapter 3). The experiments include three different datasets (PETS2006, Skating and Highway) as shown in Section 3.2.1. The details of the effect

of the history size parameter on NVSwO accuracy for PETS2006 dataset are shown in Table B.1. The history parameter has been varied using 27, 100, 300 and 900 frames, and with every value, CD threshold is varied using $10,20, \ldots, 70$. CD threshold is the value based on which the algorithm decides to consider whether a pixel value belongs to foreground or background. Bothe TPR and FPR are the statistics used to draw ROC curves (Section 3.2.1). Table B.2 shows the effect of the history parameter on TPR and FPR of SVSwS as the stride parameter is fixed at 3. Table B.3 illustrates the effect of the stride parameter on TPR and FPR of SVSwS as the history parameter remains fixed at 27. Table B.4 demonstrates the effect of the Fibonacci window size 
parameter on SVSwFW TPR and FPR. In this case, the history parameter is fixed at 900; therefore, different sample frames will be selected (e.g. window sizes 55, 89 and 610 generate 151, 106 and 27 sample frames respectively).

In the skating dataset, Table B.5 shows the effect of the history size parameter on TPR and FPR of NVSwO. Table B.6 exhibits the influence of the history size parameter on TPR and FPR of SVSwS while fixing the stride parameter at 3. The statistics in Table B.7 shows how the stride parameter affects TPR and FPR of SVSwS as the history parameter is fixed at 27. Eventually, Table B.8 demonstrates the effect of the Fibonacci window size parameter on SVSwFW TPR and FPR.

In the highway dataset, Table B.9 shows how the history size parameter influences TPR and FPR of NVSwO. The statistics in Table B.10 details the effect of the history size parameter on TPR and FPR of SVSwS when the stride parameter is fixed at 3. In Table B.11 the statistics show how the stride parameter affects TPR and FPR of SVSwS as the history parameter remains fixed at 27. Finally, Table B.12 illustrates the effect of the Fibonacci window size parameter on TPR and FPR of SVSwFW.

\section{B.2 Performance of the Proposed Sampling-based CD on a Fault Tolerant System}

This section shows the statistics of sampling-based CD under fault tolerant systems. Table B.13 and Table B.14 show the statistics for the performance of SimpleCD, $S h C D, S d C D$ and $S h d C D$ as the degree of parallelism increases in the weekday and weekend scenarios respectively. Table B.15 shows the effect of the video segment size on the execution time of the proposed algorithms in the weekday scenario, while Table B.16 captures the improvement of the execution time for the same scenario. Table 
B.17 captures the effect of the video segment size on the execution time of the proposed algorithms in the weekend scenario, and Table B.18 captures the improvement

of the execution time for the same scenario. Table B.19 and Table B.20 show the statistics for the performance of the algorithms as the number of input files increases for the weekday and weekend scenarios respectively. Table B.21 shows the execution time of the algorithms after a recovery from a node failure, while Table B.22 captures the time deterioration as the segment size increases. Table B.23 captures the effect of different point of failure on the algorithms execution time. Table B.24 captures the effect of recovery from two-node failure on the execution time of the proposed algorithms.

\section{B.3 The Experimental Results of Data Placement Policies}

This section shows the details of the proposed data placement policies. Table B.25 shows the effect of increasing the data size on the execution time and the data locality of different DPPs in the weekday scenario, while Table B.26 captures the results in the weekend scenario. Table B.27 shows the effect of the number of replicas on the execution time and the data locality of different DPPs in the weekend scenario. Table B.28 and Table B.29 show the effect of one-node failure and two-node failure on the execution time and the data locality of different DPPs respectively. 
Table B.1: Accuracy of NVSwO for the PETS2006 dataset.

\begin{tabular}{|c|c|c|c|}
\hline History & Threshold & TPR & FPR \\
\hline \multirow{7}{*}{27} & 10 & 0.769 & 0.00424 \\
\hline & 20 & 0.649 & 0.00208 \\
\hline & 30 & 0.578 & 0.00111 \\
\hline & 40 & 0.516 & 0.00057 \\
\hline & 50 & 0.482 & 0.00044 \\
\hline & 60 & 0.439 & 0.00034 \\
\hline & 70 & 0.414 & 0.00025 \\
\hline \multirow{7}{*}{100} & 10 & 0.811 & 0.00481 \\
\hline & 20 & 0.739 & 0.00254 \\
\hline & 30 & 0.654 & 0.00164 \\
\hline & 40 & 0.567 & 0.00088 \\
\hline & 50 & 0.49 & 0.00053 \\
\hline & 60 & 0.439 & 0.00047 \\
\hline & 70 & 0.412 & 0.00042 \\
\hline \multirow{7}{*}{300} & 10 & 0.881 & 0.00881 \\
\hline & 20 & 0.79 & 0.00279 \\
\hline & 30 & 0.695 & 0.00113 \\
\hline & 40 & 0.6 & 0.00068 \\
\hline & 50 & 0.512 & 0.00022 \\
\hline & 60 & 0.478 & 0.00002 \\
\hline & 70 & 0.456 & 0.00000 \\
\hline \multirow{7}{*}{900} & 10 & 0.901 & 0.00340 \\
\hline & 20 & 0.793 & 0.00086 \\
\hline & 30 & 0.715 & 0.00047 \\
\hline & 40 & 0.611 & 0.00030 \\
\hline & 50 & 0.519 & 0.00008 \\
\hline & 60 & 0.485 & 0.00002 \\
\hline & 70 & 0.466 & 0.00000 \\
\hline
\end{tabular}


Table B.2: Accuracy of SVSwS for the PETS2006 dataset (different history sizes).

\begin{tabular}{|c|c|c|c|c|}
\hline Samples & Stride & Threshold & TPR & FPR \\
\hline \multirow{7}{*}{10} & \multirow{7}{*}{3} & 10 & 0.772 & 0.00464 \\
\hline & & 20 & 0.677 & 0.00231 \\
\hline & & 30 & 0.589 & 0.00138 \\
\hline & & 40 & 0.527 & 0.00066 \\
\hline & & 50 & 0.49 & 0.00047 \\
\hline & & 60 & 0.445 & 0.00038 \\
\hline & & 70 & 0.419 & 0.00030 \\
\hline \multirow{7}{*}{27} & \multirow{7}{*}{3} & 10 & 0.796 & 0.00493 \\
\hline & & 20 & 0.726 & 0.00247 \\
\hline & & 30 & 0.654 & 0.00166 \\
\hline & & 40 & 0.567 & 0.00097 \\
\hline & & 50 & 0.5 & 0.00061 \\
\hline & & 60 & 0.46 & 0.00045 \\
\hline & & 70 & 0.432 & 0.00038 \\
\hline \multirow{7}{*}{100} & \multirow{7}{*}{3} & 10 & 0.883 & 0.00902 \\
\hline & & 20 & 0.792 & 0.00298 \\
\hline & & 30 & 0.697 & 0.00118 \\
\hline & & 40 & 0.604 & 0.00069 \\
\hline & & 50 & 0.513 & 0.00023 \\
\hline & & 60 & 0.477 & 0.00003 \\
\hline & & 70 & 0.457 & 0.00000 \\
\hline \multirow{7}{*}{300} & \multirow{7}{*}{3} & 10 & 0.901 & 0.00334 \\
\hline & & 20 & 0.794 & 0.00086 \\
\hline & & 30 & 0.716 & 0.00047 \\
\hline & & 40 & 0.617 & 0.00030 \\
\hline & & 50 & 0.519 & 0.00008 \\
\hline & & 60 & 0.485 & 0.00002 \\
\hline & & 70 & 0.467 & 0.00000 \\
\hline
\end{tabular}


Table B.3: Accuracy of SVSwS for the PETS2006 dataset (different strides).

\begin{tabular}{|c|c|c|c|c|}
\hline Samples (h') & Stride & Threshold & TPR & FPR \\
\hline \multirow{8}{*}{27} & \multirow{8}{*}{3} & 10 & 0.825 & 0.00576 \\
\hline & & 20 & 0.747 & 0.00264 \\
\hline & & 30 & 0.673 & 0.00166 \\
\hline & & 40 & 0.576 & 0.00107 \\
\hline & & 50 & 0.504 & 0.00064 \\
\hline & & 60 & 0.446 & 0.00050 \\
\hline & & 70 & 0.413 & 0.00043 \\
\hline & & 80 & 0.376 & 0.00035 \\
\hline \multirow{8}{*}{27} & \multirow{8}{*}{5} & 10 & 0.824 & 0.01980 \\
\hline & & 20 & 0.746 & 0.01020 \\
\hline & & 30 & 0.663 & 0.00492 \\
\hline & & 40 & 0.556 & 0.00240 \\
\hline & & 50 & 0.472 & 0.00059 \\
\hline & & 60 & 0.419 & 0.00036 \\
\hline & & 70 & 0.383 & 0.00025 \\
\hline & & 80 & 0.356 & 0.00018 \\
\hline \multirow{8}{*}{27} & \multirow{8}{*}{7} & 10 & 0.834 & 0.02580 \\
\hline & & 20 & 0.74 & 0.01240 \\
\hline & & 30 & 0.651 & 0.00543 \\
\hline & & 40 & 0.556 & 0.00147 \\
\hline & & 50 & 0.465 & 0.00020 \\
\hline & & 60 & 0.412 & 0.00009 \\
\hline & & 70 & 0.38 & 0.00005 \\
\hline & & 80 & 0.351 & 0.00001 \\
\hline
\end{tabular}


Table B.4: Accuracy of SVSwFW for the PETS2006 dataset.

\begin{tabular}{|c|c|c|c|c|}
\hline Window size & Sample & Threshold & TPR & FPR \\
\hline \multirow{7}{*}{55} & \multirow{7}{*}{151} & 10 & 0.867 & 0.00388 \\
\hline & & 20 & 0.782 & 0.00104 \\
\hline & & 30 & 0.69 & 0.00049 \\
\hline & & 40 & 0.566 & 0.00025 \\
\hline & & 50 & 0.505 & 0.00005 \\
\hline & & 60 & 0.468 & 0.00001 \\
\hline & & 70 & 0.443 & 0.00000 \\
\hline \multirow{7}{*}{89} & \multirow{7}{*}{106} & 10 & 0.856 & 0.00288 \\
\hline & & 20 & 0.762 & 0.00066 \\
\hline & & 30 & 0.65 & 0.00037 \\
\hline & & 40 & 0.54 & 0.00019 \\
\hline & & 50 & 0.492 & 0.00002 \\
\hline & & 60 & 0.46 & 0.00001 \\
\hline & & 70 & 0.429 & 0.00000 \\
\hline \multirow{7}{*}{610} & \multirow{7}{*}{27} & 10 & 0.904 & 0.00732 \\
\hline & & 20 & 0.791 & 0.00161 \\
\hline & & 30 & 0.715 & 0.00067 \\
\hline & & 40 & 0.631 & 0.00031 \\
\hline & & 50 & 0.537 & 0.00008 \\
\hline & & 60 & 0.491 & 0.00002 \\
\hline & & 70 & 0.469 & 0.00000 \\
\hline
\end{tabular}


Table B.5: Accuracy of NVSwO for the Skating dataset.

\begin{tabular}{|c|c|c|c|}
\hline History & Threshold & $\mathrm{TPR}$ & FPR \\
\hline \multirow{7}{*}{27} & 10 & 0.818 & 0.0671 \\
\hline & 20 & 0.745 & 0.0104 \\
\hline & 30 & 0.698 & 0.00607 \\
\hline & 40 & 0.656 & 0.00472 \\
\hline & 50 & 0.613 & 0.00369 \\
\hline & 60 & 0.568 & 0.00287 \\
\hline & 70 & 0.512 & 0.00218 \\
\hline \multirow{7}{*}{100} & 10 & 0.925 & 0.0615 \\
\hline & 20 & 0.895 & 0.0141 \\
\hline & 30 & 0.876 & 0.0089 \\
\hline & 40 & 0.861 & 0.00646 \\
\hline & 50 & 0.847 & 0.00493 \\
\hline & 60 & 0.83 & 0.00358 \\
\hline & 70 & 0.807 & 0.00238 \\
\hline \multirow{7}{*}{300} & 10 & 0.935 & 0.0628 \\
\hline & 20 & 0.907 & 0.0134 \\
\hline & 30 & 0.89 & 0.0075 \\
\hline & 40 & 0.878 & 0.00452 \\
\hline & 50 & 0.864 & 0.00269 \\
\hline & 60 & 0.849 & 0.00171 \\
\hline & 70 & 0.831 & 0.00108 \\
\hline \multirow{7}{*}{900} & 10 & 0.983 & 0.0567 \\
\hline & 20 & 0.964 & 0.00723 \\
\hline & 30 & 0.94 & 0.00327 \\
\hline & 40 & 0.918 & 0.00245 \\
\hline & 50 & 0.889 & 0.00201 \\
\hline & 60 & 0.857 & 0.00152 \\
\hline & 70 & 0.836 & 0.00106 \\
\hline
\end{tabular}


Table B.6: Accuracy of SVSwS for the skating dataset (different history sizes).

\begin{tabular}{|c|c|c|c|c|}
\hline Samples & Stride & Threshold & TPR & FPR \\
\hline \multirow{7}{*}{10} & \multirow{7}{*}{3} & 10 & 0.855 & 0.0695 \\
\hline & & 20 & 0.788 & 0.0116 \\
\hline & & 30 & 0.745 & 0.00681 \\
\hline & & 40 & 0.706 & 0.00526 \\
\hline & & 50 & 0.666 & 0.00412 \\
\hline & & 60 & 0.624 & 0.0032 \\
\hline & & 70 & 0.575 & 0.00235 \\
\hline \multirow{7}{*}{27} & \multirow{7}{*}{3} & 10 & 0.92600 & 0.06150 \\
\hline & & 20 & 0.89200 & 0.01380 \\
\hline & & 30 & 0.87300 & 0.00873 \\
\hline & & 40 & 0.85700 & 0.00651 \\
\hline & & 50 & 0.83800 & 0.00507 \\
\hline & & 60 & 0.81800 & 0.00386 \\
\hline & & 70 & 0.78900 & 0.00275 \\
\hline \multirow{7}{*}{100} & \multirow{7}{*}{3} & 10 & 0.936 & 0.0636 \\
\hline & & 20 & 0.907 & 0.0133 \\
\hline & & 30 & 0.89 & 0.00726 \\
\hline & & 40 & 0.878 & 0.00454 \\
\hline & & 50 & 0.864 & 0.0027 \\
\hline & & 60 & 0.849 & 0.00171 \\
\hline & & 70 & 0.831 & 0.00109 \\
\hline \multirow{7}{*}{300} & \multirow{7}{*}{3} & 10 & 0.984 & 0.0566 \\
\hline & & 20 & 0.963 & 0.0072 \\
\hline & & 30 & 0.941 & 0.00327 \\
\hline & & 40 & 0.92 & 0.00246 \\
\hline & & 50 & 0.892 & 0.00201 \\
\hline & & 60 & 0.86 & 0.00152 \\
\hline & & 70 & 0.836 & 0.00106 \\
\hline
\end{tabular}


Table B.7: Accuracy of SVSwS for the skating dataset (different strides).

\begin{tabular}{|c|c|c|c|c|}
\hline Samples & Stride & Threshold & TPR & FPR \\
\hline \multirow{7}{*}{27} & \multirow{7}{*}{3} & 10 & 0.92600 & 0.06150 \\
\hline & & 20 & 0.89200 & 0.01380 \\
\hline & & 30 & 0.87300 & 0.00873 \\
\hline & & 40 & 0.85700 & 0.00651 \\
\hline & & 50 & 0.83800 & 0.00507 \\
\hline & & 60 & 0.81800 & 0.00386 \\
\hline & & 70 & 0.78900 & 0.00275 \\
\hline \multirow{7}{*}{27} & \multirow{7}{*}{5} & 10 & 0.92400 & 0.06660 \\
\hline & & 20 & 0.89500 & 0.01590 \\
\hline & & 30 & 0.87700 & 0.00931 \\
\hline & & 40 & 0.86400 & 0.00677 \\
\hline & & 50 & 0.85000 & 0.00495 \\
\hline & & 60 & 0.83200 & 0.00348 \\
\hline & & 70 & 0.81300 & 0.00228 \\
\hline \multirow{7}{*}{27} & \multirow{7}{*}{7} & 10 & 0.93000 & 0.06850 \\
\hline & & 20 & 0.90000 & 0.01640 \\
\hline & & 30 & 0.88300 & 0.00905 \\
\hline & & 40 & 0.86500 & 0.00617 \\
\hline & & 50 & 0.85300 & 0.00452 \\
\hline & & 60 & 0.83800 & 0.00325 \\
\hline & & 70 & 0.81900 & 0.00207 \\
\hline
\end{tabular}


Table B.8: Accuracy of SVSwFW for the skating dataset.

\begin{tabular}{|c|c|c|c|c|}
\hline Window Size & Samples & Threshold & TPR & FPR \\
\hline \multirow{7}{*}{55} & \multirow{7}{*}{151} & 10 & 0.98463 & 0.06978 \\
\hline & & 20 & 0.97113 & 0.01462 \\
\hline & & 30 & 0.95788 & 0.01005 \\
\hline & & 40 & 0.94263 & 0.00852 \\
\hline & & 50 & 0.92250 & 0.00764 \\
\hline & & 60 & 0.89900 & 0.00677 \\
\hline & & 70 & 0.87625 & 0.00560 \\
\hline \multirow{7}{*}{89} & \multirow{7}{*}{106} & 10 & 0.98463 & 0.06910 \\
\hline & & 20 & 0.97050 & 0.01389 \\
\hline & & 30 & 0.95538 & 0.00958 \\
\hline & & 40 & 0.93738 & 0.00827 \\
\hline & & 50 & 0.91550 & 0.00746 \\
\hline & & 60 & 0.89388 & 0.00655 \\
\hline & & 70 & 0.87000 & 0.00526 \\
\hline \multirow{7}{*}{610} & \multirow{7}{*}{27} & 10 & 0.99000 & 0.10224 \\
\hline & & 20 & 0.97925 & 0.02810 \\
\hline & & 30 & 0.96738 & 0.01661 \\
\hline & & 40 & 0.95350 & 0.01071 \\
\hline & & 50 & 0.93425 & 0.00851 \\
\hline & & 60 & 0.90938 & 0.00726 \\
\hline & & 70 & 0.88588 & 0.00587 \\
\hline
\end{tabular}


Table B.9: Accuracy of NVSwO for the Highway dataset.

\begin{tabular}{|c|c|c|c|}
\hline History & Threshold & TPR & FPR \\
\hline \multirow{7}{*}{27} & 10 & 0.91100 & 0.17700 \\
\hline & 20 & 0.82500 & 0.09060 \\
\hline & 30 & 0.74500 & 0.04960 \\
\hline & 40 & 0.65500 & 0.02670 \\
\hline & 50 & 0.57000 & 0.01560 \\
\hline & 60 & 0.48900 & 0.01050 \\
\hline & 70 & 0.40700 & 0.00637 \\
\hline \multirow{7}{*}{100} & 10 & 0.93300 & 0.14300 \\
\hline & 20 & 0.86900 & 0.04750 \\
\hline & 30 & 0.79700 & 0.02010 \\
\hline & 40 & 0.73000 & 0.01030 \\
\hline & 50 & 0.65900 & 0.00558 \\
\hline & 60 & 0.57700 & 0.00301 \\
\hline & 70 & 0.49800 & 0.00160 \\
\hline \multirow{7}{*}{300} & 10 & 0.94700 & 0.45800 \\
\hline & 20 & 0.89400 & 0.06680 \\
\hline & 30 & 0.83700 & 0.02160 \\
\hline & 40 & 0.77800 & 0.00983 \\
\hline & 50 & 0.71500 & 0.00440 \\
\hline & 60 & 0.63900 & 0.00233 \\
\hline & 70 & 0.55700 & 0.00107 \\
\hline \multirow{7}{*}{900} & 10 & 0.94700 & 0.55000 \\
\hline & 20 & 0.89100 & 0.06610 \\
\hline & 30 & 0.83000 & 0.02270 \\
\hline & 40 & 0.76600 & 0.00902 \\
\hline & 50 & 0.69500 & 0.00346 \\
\hline & 60 & 0.61200 & 0.00112 \\
\hline & 70 & 0.53100 & 0.00051 \\
\hline
\end{tabular}


Table B.10: Accuracy of SVSwS for the highway dataset (different history sizes).

\begin{tabular}{|c|c|c|c|c|}
\hline Samples & Stride & Thrsh & TPR & FPR \\
\hline \multirow{7}{*}{10} & \multirow{7}{*}{3} & 10 & 0.91400 & 0.19000 \\
\hline & & 20 & 0.82800 & 0.10500 \\
\hline & & 30 & 0.74800 & 0.05930 \\
\hline & & 40 & 0.66200 & 0.02890 \\
\hline & & 50 & 0.57800 & 0.01490 \\
\hline & & 60 & 0.49600 & 0.00969 \\
\hline & & 70 & 0.41800 & 0.00574 \\
\hline \multirow{7}{*}{27} & \multirow{7}{*}{3} & 10 & 0.92800 & 0.17100 \\
\hline & & 20 & 0.85900 & 0.06280 \\
\hline & & 30 & 0.78700 & 0.03070 \\
\hline & & 40 & 0.71500 & 0.01650 \\
\hline & & 50 & 0.64200 & 0.00907 \\
\hline & & 60 & 0.55800 & 0.00459 \\
\hline & & 70 & 0.48100 & 0.00236 \\
\hline \multirow{7}{*}{100} & \multirow{7}{*}{3} & 10 & 0.94600 & 0.45500 \\
\hline & & 20 & 0.89500 & 0.06660 \\
\hline & & 30 & 0.83600 & 0.02190 \\
\hline & & 40 & 0.77800 & 0.00951 \\
\hline & & 50 & 0.71600 & 0.00428 \\
\hline & & 60 & 0.63900 & 0.00234 \\
\hline & & 70 & 0.55700 & 0.00115 \\
\hline \multirow{7}{*}{300} & \multirow{7}{*}{3} & 10 & 0.94300 & 0.51100 \\
\hline & & 20 & 0.88100 & 0.06110 \\
\hline & & 30 & 0.81200 & 0.01590 \\
\hline & & 40 & 0.74300 & 0.00612 \\
\hline & & 50 & 0.66500 & 0.00220 \\
\hline & & 60 & 0.57500 & 0.00081 \\
\hline & & 70 & 0.49600 & 0.00042 \\
\hline
\end{tabular}


Table B.11: Accuracy of SVSwS for the highway dataset (different strides).

\begin{tabular}{|c|c|c|c|c|}
\hline Samples & Stride & Threshold & TPR & FPR \\
\hline \multirow{7}{*}{27} & \multirow{7}{*}{3} & 10 & 0.92800 & 0.17100 \\
\hline & & 20 & 0.85900 & 0.06280 \\
\hline & & 30 & 0.78700 & 0.03070 \\
\hline & & 40 & 0.71500 & 0.01650 \\
\hline & & 50 & 0.64200 & 0.00907 \\
\hline & & 60 & 0.55800 & 0.00459 \\
\hline & & 70 & 0.48100 & 0.00236 \\
\hline \multirow{7}{*}{27} & \multirow{7}{*}{5} & 10 & 0.93700 & 0.17100 \\
\hline & & 20 & 0.87000 & 0.05870 \\
\hline & & 30 & 0.80200 & 0.02380 \\
\hline & & 40 & 0.72900 & 0.00884 \\
\hline & & 50 & 0.65400 & 0.00445 \\
\hline & & 60 & 0.56700 & 0.00208 \\
\hline & & 70 & 0.48600 & 0.00070 \\
\hline \multirow{7}{*}{27} & \multirow{7}{*}{7} & 10 & 0.94000 & 0.19100 \\
\hline & & 20 & 0.88000 & 0.04780 \\
\hline & & 30 & 0.81300 & 0.01980 \\
\hline & & 40 & 0.74400 & 0.00805 \\
\hline & & 50 & 0.67400 & 0.00326 \\
\hline & & 60 & 0.58800 & 0.00143 \\
\hline & & 70 & 0.50600 & 0.00079 \\
\hline
\end{tabular}


Table B.12: Accuracy of SVSwFW for the highway dataset.

\begin{tabular}{|c|c|c|c|c|}
\hline Window Size & Samples & Threshold & TPR & FPR \\
\hline \multirow{7}{*}{55} & \multirow{7}{*}{151} & 10 & 0.92700 & 0.22800 \\
\hline & & 20 & 0.86100 & 0.03770 \\
\hline & & 30 & 0.79200 & 0.01130 \\
\hline & & 40 & 0.72400 & 0.00340 \\
\hline & & 50 & 0.66200 & 0.00126 \\
\hline & & 60 & 0.58100 & 0.00059 \\
\hline & & 70 & 0.50700 & 0.00017 \\
\hline \multirow{7}{*}{89} & \multirow{7}{*}{106} & 10 & 0.92900 & 0.20500 \\
\hline & & 20 & 0.85900 & 0.03070 \\
\hline & & 30 & 0.79200 & 0.00888 \\
\hline & & 40 & 0.70800 & 0.00248 \\
\hline & & 50 & 0.61300 & 0.00092 \\
\hline & & 60 & 0.53200 & 0.00034 \\
\hline & & 70 & 0.45600 & 0.00014 \\
\hline \multirow{7}{*}{610} & \multirow{7}{*}{27} & 10 & 0.94500 & 0.49200 \\
\hline & & 20 & 0.88600 & 0.12000 \\
\hline & & 30 & 0.82300 & 0.04990 \\
\hline & & 40 & 0.75900 & 0.01880 \\
\hline & & 50 & 0.69300 & 0.00690 \\
\hline & & 60 & 0.60400 & 0.00310 \\
\hline & & 70 & 0.51800 & 0.00122 \\
\hline
\end{tabular}


Table B.13: Increasing the degree of parallelism (weekday).

\begin{tabular}{|c|c|c|c|c|c|c|}
\hline Algorithm & $\begin{array}{l}\text { No. of } \\
\text { Nodes }\end{array}$ & $\begin{array}{c}\text { Execu- } \\
\text { tion Time } \\
\quad(\text { sec.) }\end{array}$ & $\begin{array}{c}\text { Time Re- } \\
\text { duction } \\
(\%)\end{array}$ & $\begin{array}{c}\text { Parallel } \\
\text { Speedup } \\
\quad\left(S_{p}\right)\end{array}$ & $\begin{array}{l}\text { Efficiency } \\
\quad\left(E_{p}\right)\end{array}$ & $\begin{array}{l}\text { Speedup } \\
\quad(\mathrm{S})\end{array}$ \\
\hline \multirow{4}{*}{ SimpleCD } & 1 & 3947 & 100.0 & 1.0 & 1.00 & 1.0 \\
\hline & 2 & 2046 & 100.0 & 1.9 & 0.96 & 1.9 \\
\hline & 4 & 1030 & 100.0 & 3.8 & 0.96 & 3.8 \\
\hline & 8 & 546 & 100.0 & 7.2 & 0.90 & 7.2 \\
\hline \multirow{4}{*}{ ShCD } & 1 & 3731 & 94.5 & 1.0 & 1.00 & 1.1 \\
\hline & 2 & 1939 & 94.8 & 1.9 & 0.96 & 2.0 \\
\hline & 4 & 978 & 95.0 & 3.8 & 0.95 & 4.0 \\
\hline & 8 & 522 & 95.6 & 7.1 & 0.89 & 7.6 \\
\hline \multirow{4}{*}{ SdCD } & 1 & 2560 & 64.9 & 1.0 & 1.00 & 1.5 \\
\hline & 2 & 1337 & 65.3 & 1.9 & 0.96 & 3.0 \\
\hline & 4 & 680 & 66.0 & 3.8 & 0.94 & 5.8 \\
\hline & 8 & 362 & 66.3 & 7.1 & 0.88 & 10.9 \\
\hline \multirow{4}{*}{ ShdCD } & 1 & 2413 & 61.1 & 1.0 & 1.00 & 1.6 \\
\hline & 2 & 1273 & 62.2 & 1.9 & 0.95 & 3.1 \\
\hline & 4 & 643 & 62.4 & 3.8 & 0.94 & 6.1 \\
\hline & 8 & 341 & 62.5 & 7.1 & 0.88 & 11.6 \\
\hline
\end{tabular}


Table B.14: Increasing the degree of parallelism (weekend).

\begin{tabular}{|c|c|c|c|c|c|c|}
\hline Algorithm & $\begin{array}{c}\text { No. of } \\
\text { Nodes }\end{array}$ & $\begin{array}{c}\text { Execu- } \\
\text { tion Time } \\
(\text { sec. })\end{array}$ & $\begin{array}{c}\text { Time Re- } \\
\text { duction } \\
(\%)\end{array}$ & $\begin{array}{c}\text { Parallel } \\
\text { Speedup } \\
\left(S_{p}\right)\end{array}$ & $\begin{array}{c}\text { Efficiency } \\
\left(E_{p}\right)\end{array}$ & $\begin{array}{c}\text { Speedup } \\
(\mathrm{S})\end{array}$ \\
\hline \hline \multirow{4}{*}{ SimpleCD } & 1 & 4074 & 100.0 & 1.0 & 1.00 & 1.0 \\
\cline { 2 - 7 } & 2 & 2082 & 100.0 & 2.0 & 0.98 & 2.0 \\
\cline { 2 - 7 } & 4 & 1049 & 100.0 & 3.9 & 0.97 & 3.9 \\
\cline { 2 - 7 } & 8 & 544 & 100.0 & 7.5 & 0.94 & 7.5 \\
\hline \multirow{5}{*}{ ShCD } & 1 & 3848 & 94.5 & 1.0 & 1.00 & 1.1 \\
\cline { 2 - 7 } & 2 & 1967 & 94.5 & 2.0 & 0.98 & 2.1 \\
\cline { 2 - 7 } & 4 & 992 & 94.6 & 3.9 & 0.97 & 4.1 \\
\hline \multirow{5}{*}{ SdCD } & 8 & 516 & 94.9 & 7.5 & 0.93 & 7.9 \\
\cline { 2 - 7 } & 1 & 3350 & 82.2 & 1.0 & 1.00 & 1.2 \\
\cline { 2 - 7 } & 2 & 1728 & 83.0 & 1.9 & 0.97 & 2.4 \\
\cline { 2 - 7 } & 4 & 873 & 83.2 & 3.8 & 0.96 & 4.7 \\
\hline \multirow{5}{*}{ ShdCD } & 1 & 458 & 84.2 & 7.3 & 0.91 & 8.9 \\
\cline { 2 - 7 } & 2 & 1621 & 77.9 & 1.9 & 0.97 & 2.5 \\
\cline { 2 - 7 } & 4 & 822 & 78.4 & 3.8 & 0.96 & 5.0 \\
\cline { 2 - 7 } & 8 & 432 & 79.4 & 7.3 & 0.91 & 9.4 \\
\hline
\end{tabular}

Table B.15: Segment size and execution time (weekday).

\begin{tabular}{|c|c|c|c|c|}
\hline \multirow{2}{*}{ Segment Size (MB) } & \multicolumn{4}{|c|}{ Execution Time (sec.) } \\
\cline { 2 - 5 } & SimpleCD & ShCD & SdCD & ShdCD \\
\hline \hline 128 & 1044 & 981 & 704 & 664 \\
\hline 256 & 992 & 953 & 637 & 620 \\
\hline 512 & 956 & 926 & 610 & 586 \\
\hline
\end{tabular}


Table B.16: Segment size and execution time improvement (weekday).

\begin{tabular}{|c|c|c|c|c|}
\hline \multirow{2}{*}{ Segment Size (MB) } & \multicolumn{4}{|c|}{ Time Improvement (\%) } \\
\cline { 2 - 5 } & SimpleCD & ShCD & SdCD & ShdCD \\
\hline \hline 128 & 100.0 & 94.0 & 67.4 & 63.6 \\
\hline 256 & 95.0 & 91.3 & 61.0 & 59.4 \\
\hline 512 & 91.6 & 88.7 & 58.4 & 56.1 \\
\hline
\end{tabular}

Table B.17: Segment size and execution time (weekend).

\begin{tabular}{|c|c|c|c|c|}
\hline \multirow{2}{*}{ Segment Size (MB) } & \multicolumn{4}{|c|}{ Execution Time (sec.) } \\
\cline { 2 - 5 } & SimpleCD & ShCD & SdCD & ShdCD \\
\hline \hline 128 & 1072 & 1023 & 918 & 859 \\
\hline 256 & 998 & 977 & 860 & 826 \\
\hline 512 & 961 & 943 & 788 & 764 \\
\hline
\end{tabular}

Table B.18: Segment size and execution time improvement (weekend).

\begin{tabular}{|c|c|c|c|c|}
\hline \multirow{2}{*}{ Segment Size (MB) } & \multicolumn{4}{|c|}{ Time Reduction (\%) } \\
\cline { 2 - 5 } & SimpleCD & ShCD & SdCD & ShdCD \\
\hline \hline 128 & 100.0 & 95.4 & 85.6 & 80.1 \\
\hline 256 & 93.1 & 91.1 & 80.2 & 77.1 \\
\hline 512 & 89.6 & 88.0 & 73.5 & 71.3 \\
\hline
\end{tabular}

Table B.19: No. of input files and execution time (weekday).

\begin{tabular}{|c|c|c|c|c|}
\hline \multirow{2}{*}{ No. of Files } & \multicolumn{4}{|c|}{ Execution Time (sec.) } \\
\cline { 2 - 5 } & SimpleCD & ShCD & SdCD & ShdCD \\
\hline \hline 16 & 281 & 272 & 208 & 188 \\
\hline 32 & 546 & 522 & 365 & 345 \\
\hline 64 & 1035 & 982 & 695 & 646 \\
\hline
\end{tabular}


Table B.20: No. of input files and execution time (weekend).

\begin{tabular}{|c|c|c|c|c|}
\hline \multirow{2}{*}{ No. of Files } & \multicolumn{4}{|c|}{ Execution Time (sec.) } \\
\cline { 2 - 5 } & SimpleCD & ShCD & SdCD & ShdCD \\
\hline \hline 16 & 275 & 259 & 234 & 223 \\
\hline 32 & 531 & 506 & 443 & 419 \\
\hline 64 & 1084 & 1024 & 904 & 846 \\
\hline
\end{tabular}

Table B.21: One node failure recovery and execution time.

\begin{tabular}{|c|c|c|c|c|}
\hline \multirow{2}{*}{ Segment Size (MB) } & \multicolumn{4}{|c|}{ Execution Time (sec.) } \\
\cline { 2 - 5 } & SimpleCD & ShCD & SdCD & ShdCD \\
\hline \hline 128 & 641 & 609 & 421 & 397 \\
\hline 256 & 720 & 694 & 472 & 441 \\
\hline 512 & 896 & 857 & 560 & 543 \\
\hline
\end{tabular}

Table B.22: One node failure recovery and execution time deterioration.

\begin{tabular}{|c|c|c|c|c|}
\hline \multirow{2}{*}{ Segment Size(MB) } & \multicolumn{4}{|c|}{ Time Deterioration (\%) } \\
\cline { 2 - 5 } & SimpleCD & ShCD & SdCD & ShdCD \\
\hline \hline 128 & 100.0 & 95.0 & 65.7 & 61.9 \\
\hline 256 & 112.3 & 108.3 & 73.6 & 68.8 \\
\hline 512 & 139.8 & 133.7 & 87.4 & 84.7 \\
\hline
\end{tabular}

Table B.23: One-node failure recovery and point of failure (PoF).

\begin{tabular}{|c|c|c|c|c|}
\hline \multirow{2}{*}{ PoF (\%) } & \multicolumn{4}{|c|}{ Execution Time (sec.) } \\
\cline { 2 - 5 } & SimpleCD & ShCD & SdCD & ShdCD \\
\hline 25 & 643 & 608 & 418 & 395 \\
\hline 50 & 641 & 609 & 421 & 397 \\
\hline 75 & 637 & 617 & 409 & 390 \\
\hline
\end{tabular}


Table B.24: Two-node failure recovery and execution time.

\begin{tabular}{|c|c|c|c|c|}
\hline \multirow{2}{*}{ Segment Size (MB) } & \multicolumn{4}{|c|}{ Execution Time (sec.) } \\
\cline { 2 - 5 } & SimpleCD & ShCD & SdCD & ShdCD \\
\hline Weekday & 652 & 617 & 430 & 406 \\
\hline Weekend & 666 & 630 & 574 & 536 \\
\hline
\end{tabular}

Table B.25: Increasing data size effect (weekday).

\begin{tabular}{|c|c|c|c|c|c|c|c|c|}
\hline \multirow{2}{*}{ Files } & \multicolumn{2}{|c|}{ Default-DPP } & \multicolumn{2}{c|}{ NED-DPP } & \multicolumn{2}{c|}{ CW-DPP } & \multicolumn{2}{|c|}{ F\&B-DPP } \\
\cline { 2 - 9 } & $\begin{array}{c}\text { Time } \\
(\mathrm{s})\end{array}$ & $\begin{array}{c}\text { Locality } \\
(\%)\end{array}$ & $\begin{array}{c}\text { Time } \\
(\mathrm{s})\end{array}$ & $\begin{array}{c}\text { Locality } \\
(\%)\end{array}$ & $\begin{array}{c}\text { Time } \\
(\mathrm{s})\end{array}$ & $\begin{array}{c}\text { Locality } \\
(\%)\end{array}$ & $\begin{array}{c}\text { Time } \\
(\mathrm{s})\end{array}$ & $\begin{array}{c}\text { Locality } \\
(\%)\end{array}$ \\
\hline \hline 16 & 198 & 80.4 & 177 & 94.1 & 175 & 94.1 & 175 & 94.1 \\
\hline 32 & 374 & 81.8 & 357 & 97.0 & 350 & 97.0 & 349 & 97.0 \\
\hline 48 & 550 & 90.5 & 514 & 98.0 & 496 & 98.0 & 494 & 98.0 \\
\hline
\end{tabular}

Table B.26: Increasing data size effect (weekend).

\begin{tabular}{|c|c|c|c|c|c|c|c|c|}
\hline \multirow{2}{*}{ Files } & \multicolumn{2}{|c|}{ Default-DPP } & \multicolumn{2}{c|}{ NED-DPP } & \multicolumn{2}{c|}{ CW-DPP } & \multicolumn{2}{c|}{ F\&B-DPP } \\
\cline { 2 - 9 } & $\begin{array}{c}\text { Time } \\
(\mathrm{s})\end{array}$ & $\begin{array}{c}\text { Locality } \\
(\%)\end{array}$ & $\begin{array}{c}\text { Time } \\
(\mathrm{s})\end{array}$ & $\begin{array}{c}\text { Locality } \\
(\%)\end{array}$ & $\begin{array}{c}\text { Time } \\
(\mathrm{s})\end{array}$ & $\begin{array}{c}\text { Locality } \\
(\%)\end{array}$ & $\begin{array}{c}\text { Time } \\
(\mathrm{s})\end{array}$ & $\begin{array}{c}\text { Locality } \\
(\%)\end{array}$ \\
\hline \hline 16 & 286 & 58.8 & 283 & 94.1 & 239 & 94.1 & 222 & 94.1 \\
\hline 32 & 492 & 71.7 & 494 & 87.9 & 464 & 97.0 & 434 & 97.0 \\
\hline 48 & 685 & 78.2 & 682 & 85.7 & 648 & 98.0 & 648 & 98.0 \\
\hline
\end{tabular}

Table B.27: The effect of the no. of replicas.

\begin{tabular}{|c|c|c|c|c|}
\hline \multirow{2}{*}{ Policy } & \multicolumn{2}{|c|}{ ShdCD } & \multicolumn{2}{c|}{ SimpleCD } \\
\cline { 2 - 5 } & Time (s) & Locality (\%) & Time (s) & Locality (\%) \\
\hline \hline Default-DPP(1-replica) & 531 & 55.3 & 535 & 57.3 \\
\hline Default-DPP(2-replica) & 511 & 76.0 & 530 & 77.3 \\
\hline Default-DPP(3-replica) & 501 & 88.0 & 526 & 92.0 \\
\hline CW-DPP(1-replica) & 428 & 96.0 & 526 & 96.0 \\
\hline F\&B-DPP(1-replica) & 418 & 96.0 & 523 & 96.0 \\
\hline
\end{tabular}


Table B.28: One-node failure recovery.

\begin{tabular}{|c|c|c|c|c|}
\hline \multirow{2}{*}{ Policy } & \multicolumn{2}{|c|}{ ShdCD } & \multicolumn{2}{c|}{ SimpleCD } \\
\cline { 2 - 5 } & Time (s) & Locality (\%) & Time (s) & Locality (\%) \\
\hline \hline Default-DPP(2-replica) & 529 & 71.8 & 736 & 75.6 \\
\hline CW-DPP(2-replica) & 525 & 84.6 & 738 & 84.6 \\
\hline F\&B-DPP(2-replica) & 509 & 88.6 & 669 & 89.7 \\
\hline
\end{tabular}

Table B.29: Two-node failure recovery.

\begin{tabular}{|c|c|c|c|c|}
\hline \multirow{2}{*}{ Policy } & \multicolumn{2}{|c|}{ ShdCD } & \multicolumn{2}{c|}{ SimpleCD } \\
\cline { 2 - 5 } & Time (s) & Locality (\%) & Time (s) & Locality (\%) \\
\hline \hline Default-DPP(3-replica) & 536 & 81.5 & 744 & 82.7 \\
\hline ECW-DPP(3-replica) & 535 & 85.2 & 737 & 87.7 \\
\hline
\end{tabular}

